Homology, Homotopy and Applications, vol.6(1), 2004, pp.363-411

\title{
DIAGONALS ON THE PERMUTAHEDRA, MULTIPLIHEDRA AND ASSOCIAHEDRA
}

\author{
SAMSON SANEBLIDZE AND RONALD UMBLE
}

(communicated by Ross Street)

\begin{abstract}
We construct an explicit diagonal $\Delta_{P}$ on the permutahedra $P$. Related diagonals on the multiplihedra $J$ and the associahedra $K$ are induced by Tonks' projection $P \rightarrow K[\mathbf{1 9}]$ and its factorization through $J$. We introduce the notion of a permutahedral set $\mathcal{Z}$ and lift $\Delta_{P}$ to a diagonal on $\mathcal{Z}$. We show that the double cobar construction $\Omega^{2} C_{*}(X)$ is a permutahedral set; consequently $\Delta_{P}$ lifts to a diagonal on $\Omega^{2} C_{*}(X)$. Finally, we apply the diagonal on $K$ to define the tensor product of $A_{\infty}$-(co)algebras in maximal generality.
\end{abstract}

\section{Introduction}

A permutahedral set is a combinatorial object generated by permutahedra $P$ and equipped with appropriate face and degeneracy operators. Permutahedral sets are distinguished from cubical or simplicial set by higher order (non-quadratic) relations among face and degeneracy operators. In this paper we define the notion of a permutahedral set and observe that the double cobar construction $\Omega^{2} C_{*}(X)$ is a naturally occurring example. We construct an explicit diagonal $\Delta_{P}: C_{*}(P) \rightarrow$ $C_{*}(P) \otimes C_{*}(P)$ on the cellular chains of permutahedra and show how to lift $\Delta_{P}$ to a diagonal on any permutahedral set. We factor Tonks' projection $\theta: P \rightarrow$ $K$ through the multiplihedron $J$ and obtain diagonals $\Delta_{J}$ on $C_{*}(J)$ and $\Delta_{K}$ on $C_{*}(K)$. We apply $\Delta_{K}$ to define the tensor product of $A_{\infty}$-(co)algebras in maximal generality; this resolves a long-standing problem in the theory of operads. Gaberdiel and Zwiebach's open string field theory [5] provides a setting in which this tensor product can be applied.

The paper is organized as follows: Sections 2 and 5 review the families of polytopes we consider. The diagonal $\Delta_{P}$ is defined in Section 3 and lifted to general permutahedral sets in Section 4. The related diagonals $\Delta_{J}$ and $\Delta_{K}$ are obtained in Section 6 and applied in Section 7 to define the tensor product of $A_{\infty}$-(co)algebras in maximal generality. Sections 5 through 7 do not depend on Section 4.

This research was funded in part by Award No. GM1-2083 of the U.S. Civilian Research and Development Foundation for the Independent States of the Former Soviet Union (CRDF) and by Award No. 99-00817 of INTAS

This research was funded in part by a Millersville University faculty research grant.

Received November 22, 2002, revised June 7, 2004; published on September 29, 2004.

2000 Mathematics Subject Classification: Primary 55U05, 52B05, 05A18, 05A19; Secondary 55P35.

Key words and phrases: Diagonal, permutahedron, multiplihedron, associahedron.

(C) 2004, Samson Saneblidze and Ronald Umble. Permission to copy for private use granted. 
The first author wishes to acknowledge conversations with Jean-Louis Loday from which our representation of the permutahedron as a subdivision of the cube emerged. The second author wishes to thank Millersville University for its generous financial support and the University of North Carolina at Chapel Hill for its kind hospitality during work on parts of this project.

\section{The Permutahedra}

Let $S_{n}$ be the symmetric group on $\underline{n}=\{1,2, \ldots, n\}$. Recall that the permutahedron $P_{n}$ is the convex hull of $n$ ! vertices $(\sigma(1), \ldots, \sigma(n)) \in \mathbb{R}^{n}, \sigma \in S_{n}[\mathbf{4}],[\mathbf{1 4}]$, [20]. As a cellular complex, $P_{n}$ is an $(n-1)$-dimensional convex polytope whose $(n-p)$-faces are indexed by (ordered) partitions $U_{1}|\cdots| U_{p}$ of $\underline{n}$. We shall define the permutahedra inductively as subdivisions of the standard $n$-cube $I^{n}$. With this representation the combinatorial connection between faces and partitions is immediately clear.

Assign the label $\underline{1}$ to the single point $P_{1}$. If $P_{n-1}$ has been constructed and $u=$ $U_{1}|\cdots| U_{p}$ is one of its faces, form the sequence $u_{*}=\left\{u_{0}=0, u_{1}, \ldots, u_{p-1}, u_{p}=\infty\right\}$ where $u_{j}=\#\left(U_{p-j+1} \cup \cdots \cup U_{p}\right), 1 \leqslant j \leqslant p-1$ and \# denotes cardinality. Define the subdivision of $I$ relative to $u$ to be

$$
I / u_{*}=I_{1} \cup I_{2} \cup \cdots \cup I_{p},
$$

where $I_{j}=\left[1-\frac{1}{2^{u_{j-1}}}, 1-\frac{1}{2^{u_{j}}}\right]$ and $\frac{1}{2^{\infty}}=0$. Then

$$
P_{n}=\bigcup_{u \in P_{n-1}} u \times I / u_{*}
$$

with faces labeled as follows (see Figures 1 and 2):

\begin{tabular}{c|c} 
Face of $u \times I / u_{*}$ & Partition of $\underline{n}$ \\
\hline$u \times 0$ & $U_{1}|\cdots| U_{p} \mid n$ \\
$u \times\left(I_{j} \cap I_{j+1}\right)$ & $U_{1}|\cdots| U_{p-j}|n| U_{p-j+1}|\cdots| U_{p}, \quad 1 \leqslant j \leqslant p-1$ \\
$u \times 1$ & $n\left|U_{1}\right| \cdots \mid U_{p}$ \\
$u \times I_{j}$ & $U_{1}|\cdots| U_{p-j+1} \cup n|\cdots| U_{p}$.
\end{tabular}

A cubical vertex of $P_{n}$ is a vertex common to both $P_{n}$ and $I^{n-1}$. Note that $u$ is a cubical vertex of $P_{n-1}$ if and only if $u \mid n$ and $n \mid u$ are cubical vertices of $P_{n}$. Thus the cubical vertices of $P_{3}$ are $1|2| 3,2|1| 3,3|1| 2$ and $3|2| 1$ since $1 \mid 2$ and $2 \mid 1$ are cubical vertices of $P_{2}$. 


$3|1| 2$
$13 \mid 2$
$1|3| 2$
$1 \mid 23$$\left\{\begin{array}{l}3|2| 1 \\ 23 \mid 1 \\ 1|2| 3\end{array} \quad \begin{array}{l}2|3| 1 \\ 2 \mid 13 \\ 2|1| 3\end{array}\right.$

Figure 1: $P_{3}$ as a subdivision of $P_{2} \times I$.

$(1,1,1)$

$(0,1,0)$

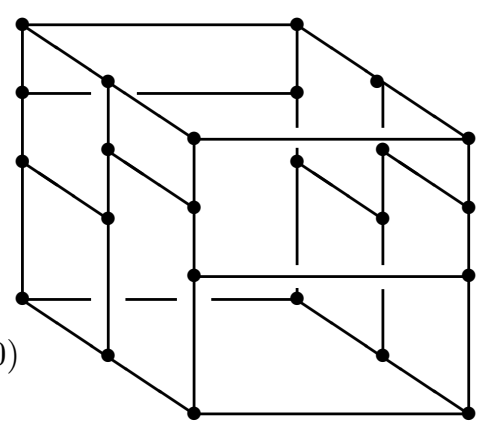

$(0,0,0) \quad(1,0,0)$

Figure 2a: $P_{4}$ as a subdivision of $P_{3} \times I$.

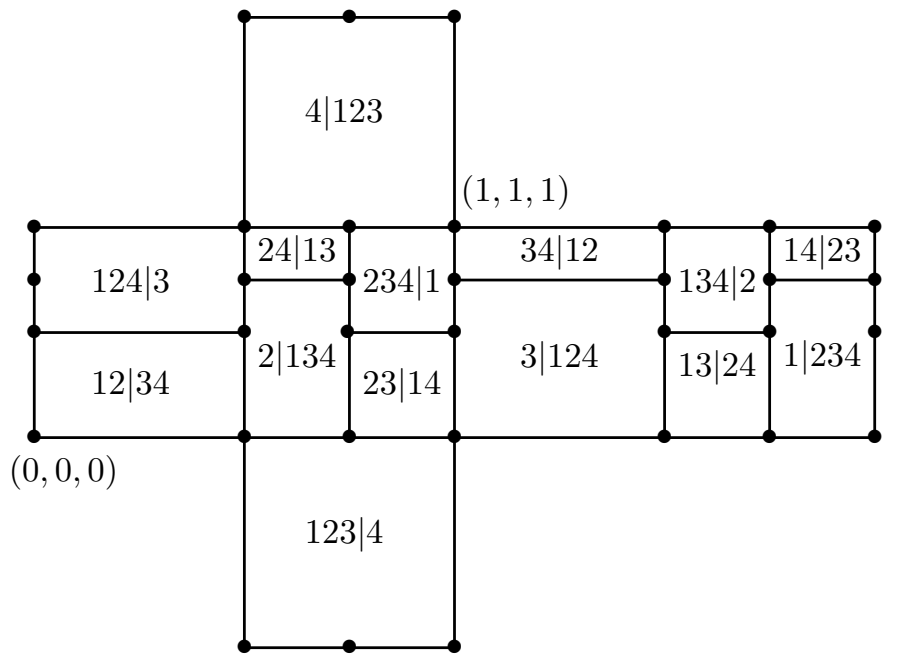

Figure 2b: The 2-faces of $P_{4}$. 


\section{A Diagonal on the Permutahedra}

In this section we construct a combinatorial diagonal on the cellular chains of the permutahedron $P_{n+1}$. Given a polytope $X$, let $\left(C_{*}(X), \partial\right)$ denote the cellular chains on $X$ with boundary $\partial$.

Definition 1. A map $\Delta_{X}: C_{*}(X) \rightarrow C_{*}(X) \otimes C_{*}(X)$ is a diagonal on $C_{*}(X)$ if

1. $\Delta_{X}\left(C_{*}(e)\right) \subseteq C_{*}(e) \otimes C_{*}(e)$ for each cell $e \subseteq X$ and

2. $\left(C_{*}(X), \Delta_{X}, \partial\right)$ is a $D G$ coalgebra.

In general, the DG coalgebra $\left(C_{*}(X), \Delta_{X}, \partial\right)$ is non-coassociative, non-cocommutative and non-counital; thus the statement (2) in Definition 1 is equivalent to stating that $\Delta_{X}$ is a chain map. We remark that a diagonal $\Delta_{P}$ on $C_{*}\left(P_{n+1}\right)$ is unique if the following two additional properties hold:

1. The canonical cellular projection $\rho_{n+1}: P_{n+1} \rightarrow I^{n}$ induces a DG coalgebra map $C_{*}\left(P_{n+1}\right) \rightarrow C_{*}\left(I^{n}\right)$ (see Section 4, Figures 3 and 4) and

2. There is a minimal number of components $a \otimes b$ in $\Delta_{P}\left(C_{k}\left(P_{n+1}\right)\right)$ for $0 \leqslant$ $k \leqslant n$.

Since the uniqueness of $\Delta_{P}$ is not used in our work, verification of these facts is left to the interested reader.

Definition 2. A partition $A_{1}|\cdots| A_{p}$ is step increasing iff $A_{p}|\cdots| A_{1}$ is step decreasing iff $\min A_{j}<\max A_{j+1}$ for all $j \leqslant p-1$. A step partition is either step increasing or step decreasing.

Think of $\sigma \in S_{p+q-1}$ as an ordered sequence of positive integers; let $\overleftarrow{\sigma}_{j}$ and $\vec{\sigma}_{q-i+1}$ denote its $j^{t h}$ decreasing and $i^{\text {th }}$ increasing subsequence of maximal length. Then $\overleftarrow{\sigma}_{1}|\cdots| \overleftarrow{\sigma}_{p}$ and $\vec{\sigma}_{q}|\cdots| \vec{\sigma}_{1}$ are step increasing and step decreasing partitions of $\underline{p+q-1}$, respectively (see Example 1 below).

Definition 3. A pairing of partitions $A_{1}|\cdots| A_{p} \otimes B_{q}|\cdots| B_{1}$ is a strong complementary pair $(S C P)$ if there exists $\sigma \in S_{p+q-1}$ such that $A_{j}=\overleftarrow{\sigma}_{j}$ and $B_{i}=\vec{\sigma}_{i}$ as unordered sets for all $i, j$.

SCP's have a natural matrix representation.

Definition 4. A $q \times p$ matrix $O=\left(o_{i j}\right)$ is ordered if:

1. $\left\{o_{i, j}\right\}=\{0,1, \ldots, p+q-1\}$;

2. Each row and column of $O$ is non-zero;

3. Non-zero entries in $O$ are distinct and increase in each row and column.

Let $\mathcal{O}$ denote the set of ordered matrices. Note that the rows and columns of an ordered matrix $O^{q \times p}$ form a partition of $\underline{p+q-1}$.

Definition 5. Given $O \in \mathcal{O}^{q \times p}$, let $V_{i}=\operatorname{row}_{i}(O) \cap \mathbb{Z}^{+}$for $i \leqslant q$ and $U_{j}=\operatorname{col}_{j}(O) \cap$ $\mathbb{Z}^{+}$for $j \leqslant p$. The row face of $O$ is the face $r(O)=V_{q}|\cdots| V_{1} \subset P_{p+q-1}$; the column face of $O$ is the face $c(O)=U_{1}|\cdots| U_{p} \subset P_{p+q-1}$. 
Definition 6. An ordered matrix $E$ is a step matrix if:

1. Non-zero entries in each row of $E$ appear in consecutive columns;

2. Non-zero entries in each column of $E$ appear in consecutive rows;

3. The sub, main and super diagonals of $E$ contain a single non-zero entry.

Let $\mathcal{E}$ denote the set of step matrices. If $E=\left(e_{i, j}\right) \in \mathcal{E}^{q \times p}$, condition (1) in Definition 6 groups the non-zero entries in each row together in a horizontal block, condition (2) groups the non-zero entries in each column together in a vertical block and condition (3) links horizontal and vertical blocks to produce a "staircase path" of non-zero entries connecting the lower-left and upper-right entries $e_{q, 1}$ and $e_{1, p}$ (see Example 1 below). Clearly, $c(E) \otimes r(E)=\overleftarrow{\sigma}_{1}|\cdots| \overleftarrow{\sigma}_{p} \otimes \vec{\sigma}_{q}|\cdots| \vec{\sigma}_{1}$ for some $\sigma \in S_{p+q-1}$, so $c(E) \otimes r(E)$ is an SCP. Furthermore, one can recover $E$ from $\sigma=\left(\begin{array}{llll}x_{1} & x_{2} & \cdots & x_{n+1}\end{array}\right)$ in the following way: Set $e_{q, 1}=x_{1}$. Inductively, assume $e_{i, j}=x_{k-1}$; if $x_{k-1}<x_{k}$, set $e_{i, j+1}=x_{k}$; otherwise, set $e_{i-1, j}=x_{k}$. Let $E_{\sigma}$ denote the step matrix given by $\sigma \in S=\lim S_{n+1}$. We have proved:

Proposition 1. There exist one-to-one correspondences

$\mathcal{E} \leftrightarrow S \leftrightarrow\{$ Step increasing partitions $\} \leftrightarrow\{$ Step decreasing partitions $\} \leftrightarrow\{S C P ' s\}$

$$
E_{\sigma} \leftrightarrow \sigma \leftrightarrow \overleftarrow{\sigma}_{1}|\cdots| \overleftarrow{\sigma}_{p} \leftrightarrow \vec{\sigma}_{q}|\cdots| \vec{\sigma}_{1} \leftrightarrow \overleftarrow{\sigma}_{1}|\cdots| \overleftarrow{\sigma}_{p} \otimes \vec{\sigma}_{q}|\cdots| \vec{\sigma}_{1}
$$

Example 1. The permutation

$$
\sigma=\left(\begin{array}{llllllll}
9 & 7 & 1 & 3 & 8 & 4 & 6 & 5
\end{array}\right)
$$

corresponds to step matrix

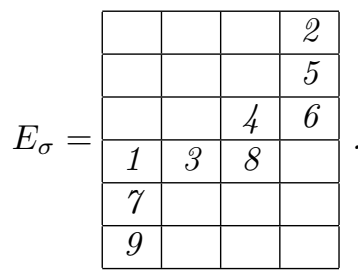

and the $S C P$

$$
c\left(E_{\sigma}\right) \otimes r\left(E_{\sigma}\right)=971|3| 84|652 \otimes 9| 7|138| 46|5| 2 .
$$

We now introduce matrix transformations that operate like the vertical and horizontal shifts one performs in a tableau puzzle. For $(i, j) \in \mathbb{Z}^{+} \times \mathbb{Z}^{+}$, define the down-shift and right-shift operators $D_{i, j}, R_{i, j}: \mathcal{O} \rightarrow \mathcal{O}$ on $O^{q \times p}=\left(o_{i, j}\right)$ by

1. $D_{i, j} O=O$ unless $i \leqslant q-1, o_{i+1, j}=0, o_{i, j} o_{i, k}>0$ for some $k \neq j, o_{i, j}>o_{i+1, \ell}$ for $1 \leqslant \ell<j$, and $o_{i+1, \ell}>o_{i, j}$ whenever $o_{i+1, \ell}>0$ and $j<\ell \leqslant p$, in which case $D_{i, j} O$ is obtained from $O$ by transposing $o_{i, j}$ and $o_{i+1, j}$;

2. $R_{i, j} O=O$ unless $j \leqslant p-1, o_{i, j+1}=0, o_{i, j} o_{k, j}>0$ for some $k \neq i, o_{i, j}>o_{\ell, j+1}$ for $1 \leqslant \ell<i$, and $o_{\ell, j+1}>o_{i, j}$ whenever $o_{\ell, j+1}>0$ and $j<\ell \leqslant q$, in which case $R_{i, j} O$ is obtained from $O$ by transposing $o_{i, j}$ and $o_{i, j+1}$. 
Definition 7. A matrix $F \in \mathcal{O}$ is a configuration matrix if there is a step matrix $E$ and a sequence of shift operators $\overline{G_{1}, \ldots, G_{m} \text { such that }}$

1. $F=G_{m} \cdots G_{1} E$;

2. If $G_{m} \cdots G_{1}=\cdots D_{i_{2}, j_{2}} \cdots D_{i_{1}, j_{1}} \cdots$, then $i_{1} \leqslant i_{2}$;

3. If $G_{m} \cdots G_{1}=\cdots R_{k_{2}, \ell_{2}} \cdots R_{k_{1}, \ell_{1}} \cdots$, then $\ell_{1} \leqslant \ell_{2}$.

When this occurs, we say that $F$ is derived from $E$ and refer to the pairing $c(F) \otimes r(F)$ as a complementary pair $(C P)$ related to $c(E) \otimes r(E)$.

Let $\mathcal{C}$ denote the set of configuration matrices. For $F=\left(f_{i, j}\right) \in \mathcal{C}$ with column face $U_{1}|\cdots| U_{p}$ and row face $V_{q}|\cdots| V_{1}$, choose proper subsets $N_{i}=\left\{f_{i, n_{1}}<\cdots\right.$ $\left.<f_{i, n_{k}} \mid \max V_{i+1}<f_{i, n_{1}}\right\} \subset V_{i}$ and $M_{j}=\left\{f_{m_{1}, j}<\cdots<f_{m_{\ell}, j} \mid \max U_{j+1}<f_{m_{1}, j}\right\}$ $\subset U_{j}$ and define

$$
D_{N_{i}}^{i} F=D_{i, n_{k}} \cdots D_{i, n_{1}} F \text { and } R_{M_{j}}^{j} F=R_{m_{\ell}, j} \cdots R_{m_{1}, j} F \text {. }
$$

We often suppress the superscript when it is clear from context. The fact that $D_{i, j+1} R_{i, j} F=R_{i+1, j} D_{i, j} F$ wherever both maps in the composition act non-trivially, gives the following useful reformulation of Definition 7:

Proposition 2. A matrix $F \in \mathcal{O}$ with $c(F)=U_{1}|\cdots| U_{p}$ and $r(F)=V_{q}|\cdots| V_{1}$ is a configuration matrix if and only if there exists $E \in \mathcal{E}$ and proper subsets $M_{j} \subset U_{j}$ and $N_{i} \subset V_{i}$ such that

$$
F=D_{N_{q-1}} \cdots D_{N_{1}} R_{M_{p-1}} \cdots R_{M_{1}} E .
$$

Example 2. Four configuration matrices $F$ can be derived from the step matrix

$$
\begin{aligned}
& E=\begin{array}{|l|l|l|}
\hline & 2 & 3 \\
\hline 1 & 5 & \\
\hline 4 & & \\
\hline
\end{array} \\
& D_{\varnothing} D_{\varnothing} R_{\varnothing} R_{\varnothing} E=\begin{array}{|l|l|l|}
\hline & 2 & 3 \\
\hline 1 & 5 & \\
\hline 4 & & \\
\hline 4
\end{array} \quad \leftrightarrow \quad 14|25| 3 \otimes 4|15| 23 \\
& D_{\varnothing} D_{\varnothing} R_{5} R_{\varnothing} E=\begin{array}{|l|l|l|}
\hline & 2 & 3 \\
\hline 1 & & 5 \\
\hline 4 & & \\
\hline
\end{array} \quad \leftrightarrow \quad 14|2| 35 \otimes 4|15| 23 \\
& D_{5} D_{\varnothing} R_{\varnothing} R_{\varnothing} E=\begin{array}{|l|l|l|}
\hline & 2 & 3 \\
\hline 1 & & \\
\hline 4 & 5 & \\
\hline
\end{array} \quad \leftrightarrow \quad 14|25| 3 \otimes 45|1| 23 \\
& D_{5} D_{\varnothing} R_{5} R_{\varnothing} E=\begin{array}{|l|l|l|}
\hline & 2 & 3 \\
\hline 1 & & \\
\hline 4 & & 5 \\
\hline
\end{array} \quad \leftrightarrow \quad 14|2| 35 \otimes 45|1| 23 .
\end{aligned}
$$


Up to sign, the CP's

$$
c(F) \otimes r(F)=(14|2| 35+14|25| 3) \otimes(4|15| 23+45|1| 23)
$$

are components of $\Delta_{P}(\underline{5})$.

Let us associate formal "configuration signs" to configuration matrices. The signs we introduce here can be derived by induction on dimension given that $P_{2}=I$ and $\Delta_{P}$ is a chain map. Henceforth we assume that all blocks in a partition are increasingly ordered. First note that a face $u=U_{1}|\cdots| U_{p} \subset P_{n+1}$ is an $(n-p+1)$ face of $p-1$ faces in dimension $n-p+2$. Thus there are $(p-1)$ ! ways to produce $u$ by successively inserting bars into $n+1$, each of which has an associated sign. Of these, we need the right-most and left-most insertion procedures.

When each $x \in \underline{n+1}$ has degree 1 , the sign of a permutation $\sigma \in S_{n+1}$ is the Koszul sign that arises from the action of $\sigma$. Thus, if $\sigma$ transposes adjacent subsets

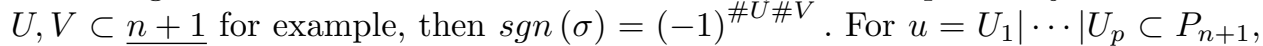
denote the sign of the permutation $\underline{n+1} \rightarrow U_{1} \cup \cdots \cup U_{p}$ by $\operatorname{psgn}(u)$; note that $\sigma$ is an unshuffle of $\underline{n}$ when $p=2$, in which case we denote $\operatorname{psgn}(u)=\operatorname{shuff}\left(U_{1} ; U_{2}\right)$. Let $m_{i}=\# U_{i}-1$ and identify $u$ with the Cartesian product $P_{m_{1}+1} \times \cdots \times P_{m_{p}+1}$; then

$$
C_{n-p+1}(u)=C_{m_{1}}\left(U_{1}\right) \otimes \cdots \otimes C_{m_{p}}\left(U_{p}\right) .
$$

Finally, think of the symbol $\mid$ as an operator with degree -1 that acts by sliding in from the left; then

$$
\left|(U \otimes V)=(-1)^{\# U} U\right| V .
$$

Definition 8. Given a partition $M \mid N$ of $\underline{n+1}$, define face operators with respect to $\underline{M \text { and } N}, d_{M}, d^{N}: C_{n}\left(P_{n+1}\right) \rightarrow C_{n-1}\left(P_{n+1}\right)$ by

$$
d_{M}(\underline{n+1})=d^{N}(\underline{n+1})=(-1)^{\# M} \operatorname{shuff}(M ; N) M \mid N .
$$

For $u=U_{1}|\cdots| U_{p} \subset P_{n+1}$ and non-empty $M \subset U_{k}$, define the face operator with respect to $M, d_{M}^{k}: C_{n-p+1}(u) \rightarrow C_{n-p}(u)$, by

$$
d_{M}^{k}(u)=\left(1^{\otimes k-1} \otimes d_{M} \otimes 1^{\otimes p-k}\right)(u) ;
$$

for $v=V_{q}|\cdots| V_{1} \subset P_{n+1}$ and non-empty $N \subset V_{k}$, define the face operator with respect to $N, d_{k}^{N}: C_{n-q+1}(v) \rightarrow C_{n-q}(v)$, by

$$
d_{k}^{N}(v)=\left(1^{\otimes q-k} \otimes d^{N} \otimes 1^{\otimes k-1}\right)(v) .
$$

Then

$$
\begin{gathered}
d_{M}^{k}(u)=\epsilon(M) U_{1}|\cdots| M\left|U_{k} \backslash M\right| \cdots \mid U_{p}, \\
d_{k}^{N}(v)=\epsilon(N) V_{q}|\cdots| V_{k} \backslash N|N| \cdots \mid V_{1},
\end{gathered}
$$

where

$$
\epsilon(M)=(-1)^{m_{1}+\cdots+m_{k-1}+\# M} \operatorname{shuff}\left(M ; U_{k} \backslash M\right) \text { and } m_{i}=\# U_{i}-1,
$$




$$
\epsilon(N)=(-1)^{n_{q}+\cdots+n_{k+1}+\#\left(V_{k} \backslash N\right)} \operatorname{shuff}\left(V_{k} \backslash N ; N\right) \text { and } n_{i}=\# V_{i}-1 .
$$

Face operators give rise to boundary operators $\partial: C_{n-p+1}(u) \rightarrow C_{n-p}(u)$ and $\partial: C_{n-q+1}(v) \rightarrow C_{n-q}(v)$ in the standard way:

$$
\partial(u)=\sum_{\substack{1 \leqslant k \leqslant p \\ M \subset U_{k}}} \epsilon(M) d_{M}^{k}\left(U_{1}|\cdots| U_{p}\right)
$$

and similarly for $\partial(v)$; in either case,

$$
\partial(\underline{n+1})=\sum_{\substack{M, N \subset \underline{n+1} \\ N=\underline{n+1} \backslash M}}(-1)^{\# M} \operatorname{shuff}(M ; N) M \mid N .
$$

The sign coefficients in 3.1 were given by R. J. Milgram in [14]. Thus, two types of signs appear when $d_{M}^{k}$ is applied to $U_{1}|\cdots| U_{p}$ : First, Koszul's sign appears when $d_{M}$ passes $U_{1} \otimes \cdots \otimes U_{k-1}$ and second, Milgram's sign appears when $d_{M}$ is applied to $U_{k}$.

A partitioning procedure is a composition of the form

$$
d_{M_{p-1}}^{k_{p-1}} \cdots d_{M_{2}}^{k_{2}} d_{M_{1}} .
$$

For example, a partition $u=U_{1}|\cdots| U_{p}$ of $\underline{n+1}$ can be obtained from the right-most partitioning procedure by setting $M_{0}=\underline{n+1}, M_{i}=M_{i-1} \backslash U_{p-i+1}$ and $k_{i}=1$ for $1 \leqslant i \leqslant p-1$; then

$$
d_{M_{p-1}}^{1} \cdots d_{M_{2}}^{1} d_{M_{1}}(\underline{n+1})=\operatorname{sgn}_{1}(u) U_{1}|\cdots| U_{p},
$$

where

$$
\operatorname{sgn}_{1}(u)=(-1)^{\epsilon_{1}} \operatorname{psgn}(u) \text { and } \epsilon_{1}=\sum_{i=1}^{p-1} i \cdot \# U_{p-i} .
$$

Note that when $v=V_{q}|\cdots| V_{1}$ we have $\epsilon_{1}=\sum_{i=1}^{q-1} i \cdot \# V_{i+1}$. Alternatively, $u$ can be obtained from the left-most partitioning procedure by setting $M_{i}=U_{i}$ and $k_{i}=i$ for $1 \leqslant i \leqslant p-1$; then

$$
d_{U_{p-1}}^{p-1} \cdots d_{U_{2}}^{2} d_{U_{1}}(\underline{n+1})=\operatorname{sgn}_{2}(u) U_{1}|\cdots| U_{p}
$$

where

$$
\operatorname{sgn}_{2}(u)=(-1)^{\epsilon_{2}} \operatorname{psgn}(u) \text { and } \epsilon_{2}=\epsilon_{1}+\left(\begin{array}{c}
p-1 \\
2
\end{array}\right) .
$$

Let $r \operatorname{sgn}\left(U_{i}\right)$ denote the sign of the order-reversing permutation on $U_{i}$, then

$$
r \operatorname{sgn}\left(U_{i}\right)=(-1)^{\frac{1}{2}\left(\# U_{i}\right)\left(\# U_{i}-1\right)} ;
$$

define

$$
r \operatorname{sgn}(u)=\prod_{i=1}^{p} r \operatorname{sgn}\left(U_{i}\right)=(-1)^{\frac{1}{2}\left[\left(\# U_{1}\right)^{2}+\cdots+\left(\# U_{p}\right)^{2}-(n+1)\right]} .
$$


Definition 9. If $F \in \mathcal{C}^{q \times p}$ is derived from $E \in \mathcal{E}$, the configuration sign of $F$ is defined to be

$$
\operatorname{csgn}(F)=(-1)^{\left(\begin{array}{l}
q \\
2
\end{array}\right)} \operatorname{rsgn}(c(E)) \cdot \operatorname{sgn}_{1} r(F) \cdot \operatorname{sgn}_{2} c(E) \cdot \operatorname{sgn}_{2} c(F) .
$$

In particular, for $F=E \in \mathcal{E}^{q \times p}$ we have

$$
\operatorname{csgn}(E)=(-1)^{\left(\begin{array}{l}
q \\
2
\end{array}\right)} r \operatorname{sgn}(c(E)) \cdot \operatorname{sgn}_{1} r(E) .
$$

Signs that arise from the action of shift operators are now determined. For $x \in \mathbb{Z}$ and $Y \subseteq \mathbb{Z}$, denote the lower and upper cuts of $Y$ at $x$ by $[Y, x)=\{y \in Y \mid y<x\}$ and $(x, Y]=\{y \in Y \mid y>x\}$, respectively.

Proposition 3. If $F=\left(f_{i, j}\right) \in \mathcal{C}, c(F)=U_{1}|\cdots| U_{p}$ and $r(F)=V_{q}|\cdots| V_{1}$, then

$$
\begin{aligned}
& \operatorname{csgn}\left(D_{i, j} F\right) \cdot \operatorname{csgn}(F)=-(-1)^{\#\left(f_{i+1, j}^{\prime}, V_{i+1}^{\prime}\right] \cup\left[V_{i}, f_{i, j}\right)}, \\
& \operatorname{csgn}\left(R_{i, j} F\right) \cdot \operatorname{csgn}(F)=-(-1)^{\#\left(f_{i, j}, U_{j}\right] \cup\left[U_{j+1}^{\prime}, f_{i, j+1}^{\prime}\right),}
\end{aligned}
$$

where $F^{\prime}=\left(f_{i, j}^{\prime}\right)$ is the image of $F, U_{1}^{\prime}|\cdots| U_{p}^{\prime}=c\left(F^{\prime}\right)$ and $V_{q}^{\prime}|\cdots| V_{1}^{\prime}=r\left(F^{\prime}\right)$.

Proof. Note that $c(F)=c\left(D_{i, j} F\right)$ and $r(F)=r\left(R_{i, j} F\right)$. Then for example,

$$
\begin{aligned}
\operatorname{csgn}\left(D_{i, j} F\right) \cdot \operatorname{csgn}(F) & =(-1)^{\left(\begin{array}{l}
q \\
2
\end{array}\right)} r \operatorname{sgn}(c(E)) \cdot \operatorname{sgn}_{1} r\left(D_{i, j} F\right) \cdot \operatorname{sgn}_{2} c(E) \cdot \operatorname{sgn}_{2} c\left(D_{i, j} F\right) \\
& \cdot(-1)^{\left(\begin{array}{l}
q \\
2
\end{array}\right)} r \operatorname{sgn}(c(E)) \cdot \operatorname{sgn}_{1} r(F) \cdot \operatorname{sgn}_{2} c(E) \cdot \operatorname{sgn}_{2} c(F) \\
& =\operatorname{sgn}_{1} r(F) \cdot \operatorname{sgn}_{1} r\left(D_{i, j} F\right) \cdot \operatorname{sgn}_{2} c(F) \cdot \operatorname{sgn}_{2} c\left(D_{i, j} F\right) \\
& =\operatorname{psgn}(r(F)) \cdot \operatorname{psgn}\left(r\left(D_{i, j} F\right)\right)=-\operatorname{sgn}(\sigma),
\end{aligned}
$$

where $\sigma$ is the permutation $V_{q} \cup \cdots \cup V_{1} \mapsto V_{q} \cup \cdots V_{i+1}^{\prime} \cup V_{i}^{\prime} \cdots \cup V_{1}$.

The configuration signs of "edge matrices," which appear in our subsequent discussion of permutahedral sets, have a particularly nice form.

Definition 10. $E \in \mathcal{E}$ is an edge matrix if $e_{1,1}=1$.

Let $\Gamma$ denote the set of all edge matrices. With one possible exception, all blocks in the column and row face of an edge matrix consist of singleton sets. Thus if $E \in \Gamma^{q \times p}$

$$
c(E) \otimes r(E)=A\left|a_{2}\right| \cdots\left|a_{p} \otimes b_{q}\right| \cdots\left|b_{2}\right| B,
$$

where $A=\left\{1<b_{2}<\cdots<b_{q}\right\}$ and $B=\left\{1<a_{2}<\cdots<a_{p}\right\}$. Since $c(E)$ and $r(E)$ meet at the cubical vertex $b_{q}|\cdots| b_{2}|1| a_{2}|\cdots| a_{p}$ of $P_{p+q-1}$, there is a canonical bijection

$$
\Gamma \leftrightarrow\left\{\text { cubical vertices of } P=\sqcup P_{n+1}\right\} \text {. }
$$

The proof of the following proposition is now immediate:

Proposition 4. If $E$ is an edge matrix and $b_{q}|\cdots| b_{2}|1| a_{2}|\cdots| a_{p}$ is the corresponding cubical vertex, then

$$
\operatorname{csgn}(E)=\operatorname{shuff}\left(b_{2}, \ldots, b_{q} ; a_{2}, \ldots, a_{p}\right) .
$$


We are ready to define a diagonal on $C_{*}\left(P_{n+1}\right)$.

Definition 11. For each $n \geqslant 0$, define $\Delta_{P}$ on the top dimensional face $\underline{n+1} \in$ $C_{n}\left(P_{n+1}\right)$ by

$$
\Delta_{P}(\underline{n+1})=\sum_{\substack{F \in \mathcal{C}^{q \times n-q+2} \\ 1 \leqslant q \leqslant n+1}} \operatorname{csgn}(F) c(F) \otimes r(F) ;
$$

extend $\Delta_{P}$ to proper faces $u=U_{1}|\cdots| U_{p} \in C_{n-p+1}(u)=C_{n_{1}}\left(U_{1}\right) \otimes \cdots \otimes C_{n_{p}}\left(U_{p}\right)$, $n_{i}=\# U_{i}-1$, via the standard comultiplicative extension.

Example 3. On $P_{3}$, all but two configuration matrices are step matrices:

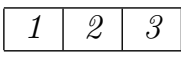

\begin{tabular}{|l|l|}
\hline & 2 \\
\hline 1 & 3 \\
\hline
\end{tabular}
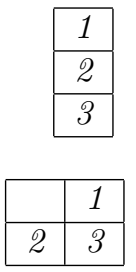
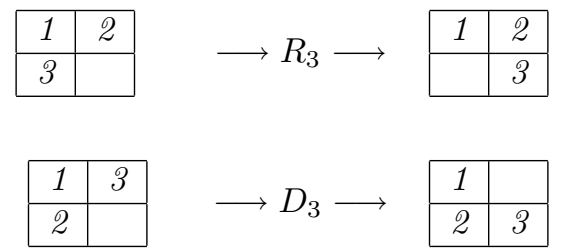

Consequently,

$$
\begin{aligned}
\Delta_{P}(\underline{3}) & =1|2| 3 \otimes 123+123 \otimes 3|2| 1 \\
& -1|23 \otimes 13| 2+2|13 \otimes 23| 1 \\
& -13|2 \otimes 3| 12+12|3 \otimes 2| 13 \\
& -1|23 \otimes 3| 12+12|3 \otimes 23| 1 .
\end{aligned}
$$

There is a computational shortcut worth mentioning. Since $F \in \mathcal{C}$ if and only if $F^{T} \in \mathcal{C}$, we only need to derive half of the configuration matrices.

Definition 12. For $F \in \mathcal{C}$, define the transpose of $c(F) \otimes r(F)$ to be

$$
[c(F) \otimes r(F)]^{T}=c\left(F^{T}\right) \otimes r\left(F^{T}\right) .
$$

Example 4. Refer to Example 3 and note that each component in the right-hand column is the transpose of the component to its left. On $P_{4}$ we have:

$$
\begin{aligned}
\Delta_{P}(\underline{4}) & =1234 \otimes 4|3| 2|1+123| 4 \otimes(3|2| 14+3|24| 1+34|2| 1) \\
& -12|34 \otimes(2|14| 3+24|1| 3)+1| 234 \otimes 14|3| 2 \\
& -23|14 \otimes(3|24| 1+34|2| 1)+13| 24 \otimes(3|14| 2+34|1| 2) \\
& +(13|24+1| 234-14|23+134| 2) \otimes 4|3| 12 \\
& -(12|34+124| 3) \otimes(4|2| 13+4|23| 1) \\
& +3|124 \otimes 34| 2|1-2| 134 \otimes(24|3| 1+4|23| 1) \\
& +24|13 \otimes 4| 23|1+(1|234-14| 23) \otimes 4| 13 \mid 2 \\
& \pm(\text { all transposes of the above). }
\end{aligned}
$$


We conclude this section with a proof of the fact that $\Delta_{P}$ is a chain map. First note that

$$
\begin{aligned}
\Delta_{P} \partial(\underline{n+1}) & =\sum \pm \Delta_{P}(M) \mid \Delta_{P}(N) \\
& =\sum \pm\left(u_{i} \otimes v_{j}\right)\left|\left(u^{k} \otimes v^{\ell}\right)=\sum \pm u_{i}\right| u^{k} \otimes v_{j} \mid v^{\ell},
\end{aligned}
$$

where $u_{i} \otimes v_{j}=c\left(F_{j \times i}\right) \otimes r\left(F_{j \times i}\right), u^{k} \otimes v^{\ell}=c\left(F^{\ell \times k}\right) \otimes r\left(F^{\ell \times k}\right)$ and $F_{j \times i}$ and $F^{\ell \times k}$ range over all configurations matrices with entries from $M$ and $N$, respectively. Although $u_{i}\left|u^{k} \otimes v_{j}\right| v^{\ell}$ is not a $\mathrm{CP}$, there is the associated block matrix

\begin{tabular}{|c|c|}
\hline 0 & $F^{\ell \times k}$ \\
\hline$F_{j \times i}$ & 0 \\
\hline
\end{tabular}

Thus the components of $\Delta_{P} \partial(\underline{n+1})$ lie in one-to-one correspondence with all such

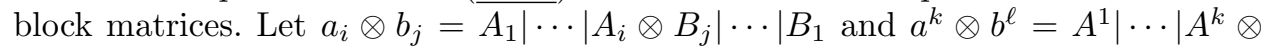
$B^{\ell}|\cdots| B^{1}$ be the SCP's related to $u_{i} \otimes v_{j}$ and $u^{k} \otimes v^{\ell}$. Denoting a column (or row) by its set of non-zero entries, the step matrices

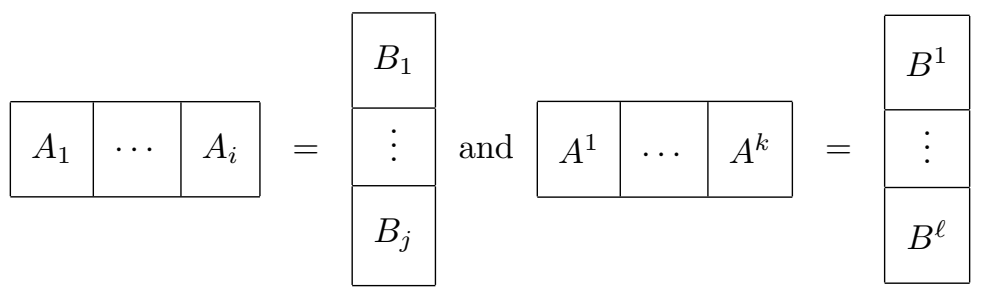

involve elements of $M$ and $N$, respectively, and the block matrix associated with the pairing $a_{i}\left|a^{k} \otimes b_{j}\right| b^{\ell}$ is

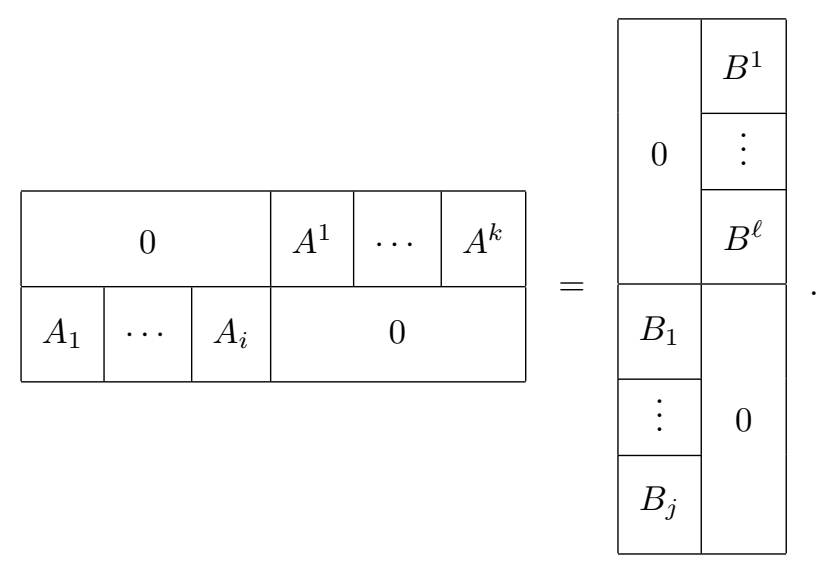

Our main result combines the statements in Lemmas 1 and 2 below:

Theorem 1. The cellular boundary map $\partial: C_{*}\left(P_{n+1}\right) \rightarrow C_{*}\left(P_{n+1}\right)$ is a $\Delta_{P^{-}}$ coderivation for all $n \geqslant 1$. 
Corollary 1. $\left(C_{*}\left(P_{n+1}\right), \Delta_{P}, \partial\right)$ is a $D G$ coalgebra and the cellular projection $\rho_{n+1}: P_{n+1} \rightarrow I^{n}$ induces a $D G$ coalgebra map

$$
\left(\rho_{n+1}\right)_{*}: C_{*}\left(P_{n+1}\right) \rightarrow C_{*}\left(I^{n}\right) .
$$

Lemma 1. Each non-zero component $\left(u_{i} \otimes v_{j}\right) \mid\left(u^{k} \otimes v^{\ell}\right)$ of $\Delta_{P} \partial \underline{(n+1)}$ is a nonzero component of $(1 \otimes \partial+\partial \otimes 1) \Delta_{P}(\underline{n+1})$.

Proof. Consider a component $\left(u_{i} \otimes v_{j}\right) \mid\left(u^{k} \otimes v^{\ell}\right)$ of $\Delta_{P} \partial(\underline{n+1})$, where $u_{i} \otimes v_{j}=$ $U_{1}|\cdots| U_{i} \otimes V_{j}|\cdots| V_{1}$ is a CP of partitions of $M=U_{1} \cup \cdots \cup U_{i}$ and $u^{k} \otimes v^{\ell}=$ $U^{1}|\cdots| U^{k} \otimes V^{\ell}|\cdots| V^{1}$ is a CP of partitions of $N=n+1 \backslash M$. The related SCP's $a_{i} \otimes b_{j}=A_{1}|\cdots| A_{i} \otimes B_{j}|\cdots| B_{1}$ and $a^{k} \otimes b^{\ell}=A^{1}\left|\overline{\cdots \mid} A^{k} \otimes B^{\ell}\right| \cdots \mid B^{1}$ give the component $\left(a_{i} \otimes b_{j}\right) \mid\left(a^{k} \otimes b^{\ell}\right)$ of $\Delta_{P} \partial(\underline{n+1})$. Let $E=\left(e_{i, j}\right)$ be the block matrix associated with $a_{i}\left|a^{k} \otimes b_{j}\right| b^{\ell}$. There are two cases:

Case 1: $e_{\ell+1, i}>e_{\ell, i+1}$.

Then $\min U_{i}=\min A_{i}>\max A^{1} \geqslant \max U^{1}$ and the $\mathrm{CP}$

$$
u \otimes v=U_{1}|\cdots| U_{i-1}\left|U^{1} \cup U_{i}\right| U^{2}|\cdots| U^{k} \otimes v_{j} \mid v^{\ell}
$$

is a component of $\Delta_{P}(\underline{n+1})$ with associated configuration matrix

$F=$\begin{tabular}{|l|l|l|l|l|}
\hline \multicolumn{2}{|c|}{0} & $U^{1}$ & $\cdots$ & $U^{k}$ \\
\hline$U_{1}$ & $\cdots$ & $U_{i}$ & \multicolumn{2}{|c|}{0} \\
\hline$v_{j}$ \\
\hline
\end{tabular}.

It follows that $u_{i} \mid u^{k} \otimes v=d_{U_{i}}^{i}(u) \otimes v$ is a component of $(1 \otimes \partial+\partial \otimes 1) \Delta_{P}(\underline{n+1})$. To check signs, we verify that the product of expressions (I) through (VI) below is 1. Let $V_{q}^{\prime}|\ldots| V_{1}^{\prime}=v_{j} \mid v^{\ell}$ and note that $u \otimes v=c(F) \otimes r(F)$ is related to the SCP $a \otimes b=A_{1}|\cdots| A_{i-1}\left|A^{1} \cup A_{i}\right| A^{2}|\cdots| A^{k} \otimes b_{j} \mid b^{\ell}=c(E) \otimes r(E)$.

I. $\operatorname{csgn}(F)=\mathrm{I}_{1} \cdot \mathrm{I}_{2} \cdot \mathrm{I}_{3} \cdot \mathrm{I}_{4} \cdot \mathrm{I}_{5}=(-1)^{\left(\begin{array}{l}q \\ 2\end{array}\right)} \cdot\left[\operatorname{sgn}_{2} u \cdot \operatorname{sgn}_{2} a\right] \cdot r \operatorname{sgn}(a) \cdot(-1)^{\epsilon_{1}^{\prime}}$. $\operatorname{psgn}(v)$, where $\epsilon_{1}^{\prime}=\sum_{i=1}^{q-1} i \cdot \# V_{i+1}^{\prime}$.

II. $\operatorname{sgn}\left(d_{U_{i}}^{i}(u)\right)=\mathrm{II}_{1} \cdot \mathrm{II}_{2}=(-1)^{\# M+i+1} \cdot(-1)^{\# U_{i} \# U^{1}}$, where the shuffle sign $\mathrm{II}_{2}$ follows by assumption.

III. $\operatorname{sgn}\left(d_{M}(\underline{n+1})\right)=\mathrm{III}_{1} \cdot \mathrm{III}_{2}=(-1)^{\# M} \cdot \operatorname{shuff}(M ; N)$.

IV. $\operatorname{csgn}\left(F_{j \times i}\right)=\mathrm{IV}_{1} \cdot \mathrm{IV}_{2} \cdot \mathrm{IV}_{3} \cdot \mathrm{IV}_{4} \cdot \mathrm{IV}_{5}=(-1)^{\left(\begin{array}{l}j \\ 2\end{array}\right)} \cdot\left[\operatorname{sgn}_{2} u_{i} \cdot \operatorname{sgn}_{2} a_{i}\right] \cdot r \operatorname{sgn}\left(a_{i}\right) \cdot$ $(-1)^{\epsilon_{1}} \cdot \operatorname{psgn}\left(v_{j}\right)$, where $\epsilon_{1}=\sum_{i=1}^{j-1} i \cdot \# V_{i+1}$.

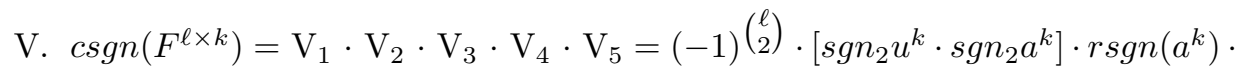
$(-1)^{\epsilon^{1}} \cdot \operatorname{psgn}\left(v^{\ell}\right)$, where $\epsilon^{1}=\sum_{i=1}^{\ell-1} i \cdot \# V^{i+1}$.

VI. $(-1)^{\operatorname{dim} u^{k} \operatorname{dim} v_{j}}=(-1)^{(\ell-1)(i-1)}\left(u_{i} \otimes v_{j}\right.$ is a component of $\Delta_{P}(M)$; hence $\operatorname{dim}\left(u_{i} \otimes v_{j}\right)=\# M-1$ and $\left.\operatorname{dim} v_{j}=\# M-1-\operatorname{dim} u_{i}=i-1\right)$. 
Then by straightforward calculation,

(1) $\mathrm{I}_{5} \cdot \mathrm{III}_{2} \cdot \mathrm{IV}_{5} \cdot \mathrm{V}_{5}=1$;

(2) $\mathrm{I}_{2} \cdot \mathrm{IV}_{2} \cdot \mathrm{V}_{2}=\mathrm{I}_{3} \cdot \mathrm{II}_{2} \cdot \mathrm{IV}_{3} \cdot \mathrm{V}_{3}=(-1)^{\# A_{i} \# A^{1}+\# U_{i} \# U^{1}}$;

(3) $\mathrm{I}_{1} \cdot \mathrm{IV}_{1} \cdot \mathrm{V}_{1}=\left(\mathrm{I}_{4} \cdot \mathrm{IV}_{4} \cdot \mathrm{V}_{4}\right) \cdot\left(\mathrm{II}_{1} \cdot \mathrm{III}_{1}\right) \cdot \mathrm{VI}=(-1)^{j \ell}$

$\left(\# M=i+j-1\right.$ since $\left.v_{j}=r\left(F_{j \times i}\right)\right)$.

Case 2: $e_{\ell+1, i}<e_{\ell, i+1}$.

Then $\max \left(V_{1}\right) \leqslant \max \left(B_{1}\right)<\min \left(B^{\ell}\right)=\min \left(V^{\ell}\right)$ and the CP

$$
u \otimes v=u_{i}\left|u^{k} \otimes V_{j}\right| \cdots\left|V_{1} \cup V^{\ell}\right| \cdots \mid V^{1}
$$

is a component of $\Delta_{P}(\underline{n+1})$ with associated configuration matrix

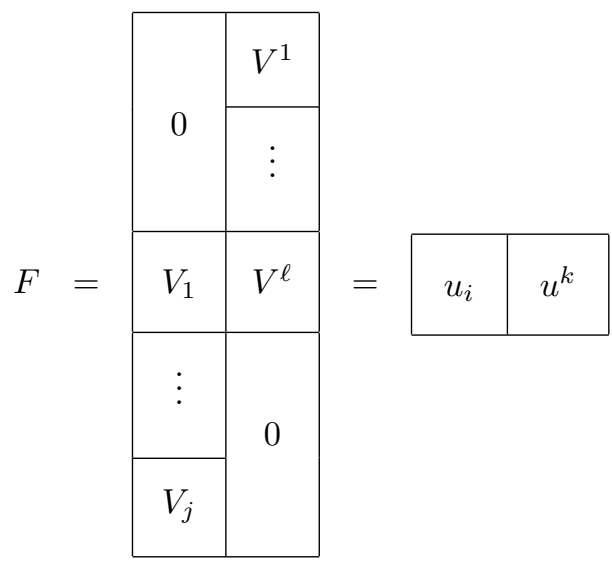

It follows that $u_{i}\left|u^{k} \otimes v_{j}\right| v^{\ell}=u \otimes d_{\ell}^{V_{1}}(v)$ is a component of $(1 \otimes \partial+\partial \otimes 1) \Delta_{P}(\underline{n+1})$. The sign check is similar to the one in Case 1 above and is left to the reader.

Lemma 2. Each non-zero component $d_{M}^{k}(u) \otimes v$ or $u \otimes d_{\ell}^{N}(v)$ of $(1 \otimes \partial+\partial \otimes 1)$ $\Delta_{P}(\underline{n+1})$ is a non-zero component of $\Delta_{P} \partial(\underline{n+1})$.

Proof. For simplicity we work with $\mathbb{Z}_{2}$ coefficients; sign checks with $\mathbb{Z}$ coefficients are straightforward calculations and left to the reader. Given an SCP $a \otimes b=c(E) \otimes$ $r(E)=A_{1}\left|\cdots A_{p} \otimes B_{q}\right| \cdots \mid B_{1}$ of partitions of $n+1$, let $u \otimes v=c(F) \otimes r(F)=$ $U_{1}|\cdots| U_{p} \otimes V_{q}|\cdots| V_{1}$ be a related CP. Then there exist $M_{j} \subset A_{j}$ and $N_{i} \subset B_{i}$ with $\min M_{j}>\max A_{j+1}$ and $\min N_{i}>\max B_{i+1}$ such that

$$
F=D_{N_{q-1}} \cdots D_{N_{1}} R_{M_{p-1}} \cdots R_{M_{1}} E .
$$

Then $u \otimes v$ is a non-zero component of $\Delta_{P}(\underline{n+1})$. For each proper $M \subset U_{k}$, we prove that the component $d_{M}^{k}(u) \otimes v$ of $(1 \otimes \partial+\partial \otimes 1) \Delta_{P}(\underline{n+1})$ is a non-zero component of $\Delta_{P} \partial(\underline{n+1})$ if and only if the following conditions hold:

(1) $m=\min M \in A_{k}$; 
(2) $(m, M]=\left(m, A_{k} \cup M_{k-1}\right]$;

(3) $m \in B_{r}$ implies $N_{r-1}=\varnothing$.

The dual statement for $u \otimes d_{\ell}^{N}(v)$ with $N \subset V_{\ell}$ and is also true; the proof follows by "mirror symmetry." Suppose conditions (1) - (3) hold. Set $M_{0}=M_{p}=\varnothing$; then clearly, $U_{i}=\left(A_{i} \cup M_{i-1}\right) \backslash M_{i}$ for $1 \leqslant i \leqslant p$, and $M_{k-1} \subseteq M$ by conditions (1) and (2). Thus $U_{1} \cup \cdots \cup U_{k-1} \cup M=A_{1} \cup \cdots \cup A_{k-1} \cup M$ and it follows that $d_{M}^{k}(u) \otimes v$ is the non-zero component

$$
\Delta_{P}\left(A_{1} \cup \cdots \cup A_{k-1} \cup M \mid A_{k} \backslash M \cup A_{k+1} \cup \cdots \cup A_{p}\right)
$$

of $\Delta_{P} \partial(\underline{n+1})$. Conversely, if conditions (1) - (3) fail to hold, we prove that there exists a unique $\mathrm{CP} \bar{u} \otimes \bar{v} \neq u \otimes v$ such that $u \otimes v+\bar{u} \otimes \bar{v} \in \operatorname{ker}(\partial \otimes 1+1 \otimes \partial)$.

For existence, we consider all possible cases.

Case 1: Assume $(1)^{\prime}: m \notin A_{k}$.

Let

$$
\bar{u}=U_{1}|\cdots| U_{k-1} \cup M\left|U_{k} \backslash M\right| \cdots \mid U_{p} ;
$$

then

$$
d_{U_{k-1}}^{k-1}(\bar{u}) \otimes v=d_{M}^{k}(u) \otimes v .
$$

Now $M \subset M_{k-1}$ since $m \in M_{k-1}$; hence $\bar{u} \otimes v$ may be obtained by replacing $R_{M_{k-1}}$ with $R_{M_{k-1} \backslash M}$ in (3.4) and $\bar{u} \otimes v$ is a CP related to $a \otimes b$.

Case 2: Assume $(1) \wedge(2)^{\prime}: m \in A_{k}$ and $(m, M] \subset\left(m, A_{k} \cup M_{k-1}\right]$.

Let

$$
\mu=\min \left(m, A_{k} \cup M_{k-1}\right] \backslash M \text { and } L=\left[A_{k}, m\right) \cup \mu .
$$

Note that $\mu \in A_{i}$ for some $1 \leqslant i \leqslant k$.

Subcase 2A: Assume $\min L>\max A_{k+1}, k<p$.

Let

$$
\bar{u}=U_{1}|\cdots| M\left|\left(U_{k} \backslash M\right) \cup U_{k+1}\right| \cdots \mid U_{p} ;
$$

then

$$
d_{U_{k} \backslash M}^{k+1}(\bar{u}) \otimes v=d_{M}^{k}(u) \otimes v .
$$

Note that $\min A_{k}=m$ since $\min L>\max A_{k+1}>\min A_{k}$. Thus $L=\mu$. Now, $\min M_{k}>\max A_{k+1}$ by (3.4) and $\min U_{k} \backslash M=\min \left[\left(A_{k} \cup M_{k-1}\right) \backslash M_{k}\right] \backslash M \geqslant$ $\min \left(A_{k} \cup M_{k-1}\right) \backslash M=\min \left(m, A_{k} \cup M_{k-1}\right] \backslash M=\mu=\min L>\max A_{k+1}$ so that $\min M_{k} \cup\left(U_{k} \backslash M\right)>\max A_{k+1}$. Hence $\bar{u} \otimes v$ can be obtained by replacing $R_{M_{k}}$ with $R_{M_{k} \cup\left(U_{k} \backslash M\right)}$ in (3.4) and $\bar{u} \otimes v$ is a CP related to $a \otimes b$.

Subcase 2B: $\min L<\max A_{k+1}$ with $k \leqslant p$. 
Subcase 2B1: Assume $\min A_{i-1}>\max A_{i} \backslash \mu$ with $\mu \in A_{i}$ and $1<i \leqslant k$.

When $i=k$ let

$$
\bar{u}=U_{1}|\cdots| U_{k-1} \cup M\left|U_{k} \backslash M\right| \cdots \mid U_{p} ;
$$

and when $1<i<k$, let

$$
\bar{u}=U_{1}|\cdots| U_{i-1} \cup U_{i}|\cdots| M\left|U_{k} \backslash M\right| \cdots \mid U_{p} .
$$

Then for all $i \leqslant k$,

$$
d_{M}^{k}(u) \otimes v=d_{U_{i-1}}^{i-1}(\bar{u}) \otimes v .
$$

When $i=k, \min A_{k-1} \cup\left(A_{k} \cap M\right) \leqslant \min A_{k} \cap M<\mu=\max A_{k}=\max A_{k} \backslash M$ so that

$$
\bar{a} \otimes \bar{b}=A_{1}|\cdots| A_{k-1} \cup\left(A_{k} \cap M\right)\left|A_{k} \backslash M\right| \cdots \mid A_{p} \otimes b
$$

is an SCP; let $\bar{E}$ be the associated step matrix and let

$$
\bar{F}=D_{N_{q-1}} \cdots D_{N_{1}} R_{M_{p-1}} \cdots R_{M_{k}} R_{M_{k-1} \backslash M} \cdots R_{M_{1}} \bar{E} .
$$

When $i<k$, we have $\mu=\max A_{i}>\max A_{k} \geqslant \max A_{k} \cap M$ so that $\min A_{k} \cap$ $M<\max L$; furthermore, $\max A_{k}=\max A_{k} \cap M$ by the minimality of $\mu$ so that $\min A_{k-1}<\max A_{k} \cap M$. And finally, $\min L<\max A_{k+1}$ by assumption $2 \mathrm{~B}$. Thus

$$
\begin{aligned}
\bar{a} \otimes \bar{b}=A_{1}|\cdots| A_{i-1} \cup A_{i} \backslash \mu \mid & \cdots\left|A_{k} \cap M\right| L|\cdots| A_{p} \\
& \otimes B_{q}|\cdots| B_{r+1}\left|B_{r-1}\right| \cdots\left|B_{j} \cup \mu\right| \cdots \mid B_{1}
\end{aligned}
$$

is an SCP; let $\bar{E}$ be the associated step matrix. Note that $U_{i-1} \cup U_{i}=$ $\left(A_{i-1} \cup M_{i-2} \cup A_{i} \backslash \mu\right) \backslash\left(M_{i} \backslash \mu\right)$ and $\mu \in M_{j}$ for $i \leqslant j \leqslant k-1$. Let

$$
\begin{aligned}
\bar{F}= & D_{N_{q-1}} \cdots D_{N_{r-1} \cup \mu} \cdots D_{N_{j} \cup \mu} \cdots D_{N_{1}} \\
& R_{M_{p-1}} \cdots R_{M_{k}} R_{\left(M_{k-1} \backslash \mu\right) \backslash M}^{k-1} R_{M_{k-1} \backslash \mu}^{k-2} \cdots R_{M_{i} \backslash \mu}^{i-1} \cdots R_{M_{1}} \bar{E},
\end{aligned}
$$

where $\mu \in B_{r}, \bar{B}_{j}$. Then for all $i \leqslant k, \bar{u} \otimes v=c(\bar{F}) \otimes r(\bar{F})$ is a CP related to $\bar{a} \otimes \bar{b}$.

Subcase 2B2: Assume $\min A_{i-1}<\max A_{i} \backslash \mu$ with $\mu \in A_{i}$ and $1<i \leqslant k$.

Let

$$
\bar{u} \otimes \bar{v}=U_{1}|\cdots| M\left|U_{k} \backslash M\right| \cdots\left|U_{p} \otimes V_{q}\right| \cdots\left|V_{r} \cup V_{r-1}\right| \cdots \mid V_{1},
$$

where $\mu \in B_{r}, \bar{B}_{j}$. Then

$$
d_{M}^{k}(u) \otimes v=\bar{u} \otimes d_{r-1}^{V_{r-1}}(\bar{v})
$$

When $i=k, \max L=\mu \in A_{k}$ so that $\min A_{k-1}<\max A_{k} \backslash \mu=\max A_{k} \backslash L$. Furthermore, $\min A_{k} \backslash L=m<\mu=\max L$; and finally, $\min L<\max A_{k+1}$ by assumption 2B. Thus

$$
\bar{a} \otimes \bar{b}=A_{1}|\cdots| M|L| \cdots\left|A_{p} \otimes B_{q}\right| \cdots\left|B_{r+1}\right| B_{r-1}|\cdots| B_{j} \cup \mu|\cdots| B_{1}
$$


is an SCP; let $\bar{E}$ be the associated step matrix. Since $\min \left(\mu, A_{k} \cup M_{k-1}\right] \backslash M>\mu=$ $\max L$, the operator $R_{\left(\mu, A_{k} \cup M_{k-1} \backslash \backslash M\right.}$ is defined. Note that $M_{k} \subset L \cup\left(\mu, A_{k} \cup M_{k-1}\right] \backslash$ $M$ and let

$$
\begin{aligned}
\bar{F}=D_{N_{q-1}}^{q-2} \cdots D_{N_{r}}^{r-1} D_{N_{r-2} \cup \mu} \cdots D_{N_{j} \cup \mu} \cdots D_{N_{1}} \\
R_{M_{p-1}}^{p} \cdots R_{M_{k}}^{k+1} R_{\left(\mu, A_{k} \cup M_{k-1} \backslash \backslash M\right.}^{k} \cdots R_{M_{1}} \bar{E} .
\end{aligned}
$$

When $1<i<k$ we have min $A_{i-1}<\max A_{i} \backslash \mu$ by assumption 2B2, and $\min A_{i} \backslash \mu<$ $\max A_{i+1}$ since $\mu \in A_{i} \cap M_{k-1}$ implies $\mu>\min A_{i}$. Next, $\min A_{k-1}<\max A_{k}=$ $\max A_{k} \cap M$ since $\max A_{k}<\mu \in A_{i}$, and $\min A_{k} \cap M<\mu=\max L$. Finally, $\min L<\max A_{k+1}$ by assumption $2 \mathrm{~B}$. Thus

$$
\begin{aligned}
\bar{a} \otimes \bar{b}=A_{1}|\cdots| A_{i} \backslash \mu|\cdots| A_{k} & \cap M|L| \cdots \mid A_{p} \\
& \otimes B_{q}|\cdots| B_{r+1}\left|B_{r-1}\right| \cdots\left|B_{j} \cup \mu\right| \cdots \mid B_{1}
\end{aligned}
$$

is an SCP; let $\bar{E}$ be the associated step matrix. Since $\min M_{k-1}=\min M_{k-1} \backslash M=$ $\mu>\max A_{k}$, both $R_{M_{k-1} \backslash \mu}$ and $R_{\left(M_{k-1} \backslash \mu\right) \backslash M}$ are defined, so let

$$
\begin{aligned}
\bar{F}= & D_{N_{q-1}}^{q-2} \cdots D_{N_{r}}^{r-1} D_{N_{r-2} \cup \mu} \cdots D_{N_{j} \cup \mu} \cdots D_{N_{1}} \\
& R_{M_{p-1}}^{p} \cdots R_{M_{k}}^{k+1} R_{\left(M_{k-1} \backslash \mu\right) \backslash M}^{k} R_{M_{k-1} \backslash \mu} \cdots R_{M_{i} \backslash \mu} \cdots R_{M_{1}} \bar{E} .
\end{aligned}
$$

Then for all $i \leqslant k, \bar{u} \otimes v=c(\bar{F}) \otimes r(\bar{F})$ is a CP related to $\bar{a} \otimes \bar{b}$.

Case 3: Assume $(1) \wedge(2) \wedge(3)^{\prime}: m \in A_{k} \cap B_{r},(m, M]=\left(m, A_{k} \cup M_{k-1}\right]$ and $N_{r-1} \neq \varnothing$.

Note that $M_{k} \subset\left(A_{k} \cup M_{k-1}\right) \backslash M=\left[A_{k}, m\right)$ by conditions (1) and (2) so that $U_{k} \backslash M=\left[A_{k}, m\right) \backslash M_{k}$. Let $\nu=\min N_{r-1}$; then $\nu \in B_{i} \cap A_{j}$ for some $1 \leqslant i \leqslant r-1$ and

$$
j=k+\#\left[B_{i}, \nu\right)+\sum_{s=i+1}^{r-1}\left(\# B_{s}-1\right) .
$$

Subcase 3A: Assume $A_{j}=\nu$.

In subcases $3 \mathrm{~A} 1$ and $3 \mathrm{~A} 2, \bar{u}$ is defined so that

$$
d_{\left[A_{k}, m\right)}^{k+1}(\bar{u}) \otimes v=d_{M}^{k}(u) \otimes v .
$$

Subcase 3A1: $j=k+1$.

Let

$$
\bar{u}=U_{1}|\cdots| M\left|\left(U_{k} \backslash M\right) \cup U_{k+1}\right| U_{k+2}|\cdots| U_{p} .
$$

But $\nu>m$ since $\nu \in A_{k+1} \cap N_{r-1}$, consequently $M_{k}=\varnothing$ so that $U_{k} \backslash M=\left[A_{k}, m\right)$ and $U_{k+1}=A_{k+1}=\nu$; thus $M_{k+1}=\varnothing$. Clearly

$$
\begin{aligned}
\bar{a} \otimes \bar{b}=A_{1}|\cdots| A_{k} \cap M \mid\left[A_{k}, m\right) & \cup \nu\left|A_{k+2}\right| \cdots \mid A_{p} \\
& \otimes B_{q}|\cdots| B_{r} \cup \nu|\cdots| B_{i} \backslash \nu|\cdots| B_{1}
\end{aligned}
$$


is an SCP; let $\bar{E}$ be the associated step matrix and let

$$
\bar{F}=D_{N_{q-1}} \cdots D_{N_{r-1} \backslash \nu} \cdots D_{N_{i} \backslash \nu} \cdots D_{N_{1}} R_{M_{p-1}} \cdots R_{\varnothing}^{k+1} R_{\varnothing}^{k} \cdots R_{M_{1}} \bar{E} ;
$$

then $\bar{u} \otimes v=c(\bar{F}) \otimes r(\bar{F})$ is a CP related to $\bar{a} \otimes \bar{b}$.

Subcase 3A2: $j>k+1$.

Let

$$
\bar{u}=U_{1}|\cdots| M\left|U_{k} \backslash M\right| \cdots\left|U_{j-1} \cup U_{j}\right| U_{j+1}|\cdots| U_{p} .
$$

Again, $\nu>m$ implies that $M_{j-1}=\varnothing$ and $U_{j}=A_{j}=\nu$. Clearly

$$
\begin{aligned}
\bar{a} \otimes \bar{b}=A_{1}|\cdots| A_{k} \cap M\left|\left[A_{k}, m\right) \cup \nu\right| \cdots\left|A_{j-1}\right| A_{j+1}|\cdots| A_{p} \\
\otimes B_{q}|\cdots| B_{r} \cup \nu|\cdots| B_{i} \backslash \nu|\cdots| B_{1}
\end{aligned}
$$

is an SCP; let $\bar{E}$ be the associated step matrix and let

$$
\begin{aligned}
\bar{F}= & D_{N_{q-1}} \cdots D_{N_{r-1} \backslash \nu} \cdots D_{N_{i} \backslash \nu} \cdots D_{N_{1}} \\
& \quad R_{M_{p-1}} \cdots R_{\varnothing}^{j} R_{M_{j-2} \cup \nu}^{j-1} \cdots R_{M_{k+1} \cup \nu}^{k+2} R_{M_{k} \cup \nu}^{k+1} R_{\varnothing}^{k} \cdots R_{M_{1}} \bar{E} ;
\end{aligned}
$$

then $\bar{u} \otimes v=c(\bar{F}) \otimes r(\bar{F})$ is a CP related to $\bar{a} \otimes \bar{b}$.

Subcase $3 B$ : Assume $A_{j} \neq \nu$.

Note that $i>1$ by assumption and let

$$
\bar{u} \otimes \bar{v}=U_{1}|\cdots| M\left|U_{k} \backslash M\right| \cdots\left|U_{p} \otimes V_{q}\right| \cdots\left|V_{i} \cup V_{i-1}\right| \cdots \mid V_{1} ;
$$

then

$$
d_{M}^{k}(u) \otimes v=\bar{u} \otimes d_{i-1}^{V_{i-1}}(\bar{v}) .
$$

Note that $\nu>m$ implies $M_{j-1}=\varnothing$ and $U_{j}=A_{j} \backslash M_{j}$. Clearly

$$
\begin{aligned}
& \bar{a} \otimes \bar{b}=A_{1}|\cdots| A_{k} \cap M\left|\left[A_{k}, m\right) \cup \nu\right| \cdots\left|A_{j-1}\right| A_{j} \backslash \nu\left|A_{j+1}\right| \cdots \mid A_{p} \\
& \otimes B_{q}|\cdots| B_{r} \cup \nu|\cdots|\left(B_{i} \cup B_{i-1}\right) \backslash \nu|\cdots| B_{1}
\end{aligned}
$$

is a SCP; let $\bar{E}$ be the associated step matrix and let

$$
\begin{aligned}
\bar{F}= & D_{N_{q-1}} \cdots D_{N_{r-1} \backslash \nu} \cdots D_{N_{i} \backslash \nu} \cdots D_{N_{1}} \\
& R_{M_{p-1}}^{p} \cdots R_{M_{j}}^{j+1} R_{M_{j-1} \cup \nu}^{j} \cdots R_{M_{k} \cup \nu}^{k+1} R_{\varnothing}^{k} \cdots R_{M_{1}} \bar{E} ;
\end{aligned}
$$

then $\bar{u} \otimes \bar{v}=c(\bar{F}) \otimes r(\bar{F})$ is a CP related to $\bar{a} \otimes \bar{b}$.

For uniqueness of each pair $\bar{u} \otimes \bar{v}$ constructed above, note the transformations $R$ and $D$ fix minimal elements, i.e., if $\bar{u} \otimes \bar{v}=R(\bar{a}) \otimes D(\bar{b})$, then necessarily $\min \bar{U}_{i}=$ $\min \bar{A}_{i}$ and $\min \bar{V}_{i}=\min \bar{B}_{i}$ for all $i$; in particular, if $R(\bar{a})=\tilde{R}\left(a^{\prime}\right)$ or $D(\bar{b})=\tilde{D}\left(b^{\prime}\right)$ then $\min \bar{A}_{i}=\min A_{i}^{\prime}$ or $\min \bar{B}_{i}=\min B_{i}^{\prime}$. Consequently, for $d_{M}^{k}(u) \otimes v$ or $u \otimes d_{\ell}^{N}(v)$ in the cases above, there is exactly one way to construct a step matrix $\bar{E}$ so that $\bar{a}$ is step increasing and $\bar{b}$ is step decreasing (it is straightforward to check that a construction with distinct $u \otimes v, \bar{u} \otimes \bar{v}$, and $u^{\prime} \otimes v^{\prime}$ would contradict the necessary condition above either for $a$ and $a^{\prime}$ or for $b$ and $b^{\prime}$ ). This completes the proof. 


\section{Permutahedral Sets}

This section introduces the notion of a permutahedral set $\mathcal{Z}$, which is a combinatorial object generated by permutahedra and equipped with appropriate face and degeneracy operators. We construct the generating category $\mathbf{P}$ and show how to lift the diagonal on the permutahedra $P$ constructed above to a diagonal on $\mathcal{Z}$. Naturally occurring examples of permutahedral sets include the double cobar construction, i.e., Adams' cobar construction [1] on the cobar with coassociative coproduct $[\mathbf{2}],[\mathbf{3}],[\mathbf{8}]$ (see Subsection 4.5 below). Permutahedral sets are distinguished from simplicial or cubical sets by their higher order structure relations. While our construction of $\mathbf{P}$ follows the analogous (but not equivalent) construction for polyhedral sets given by D.W. Jones in [7], there is no mention of structure relations in $[7]$.

\subsection{Singular Permutahedral Sets}

By way of motivation we begin with constructions of two singular permutahedral sets-our universal examples. Whereas the first emphasizes coface and codegeneracy operators, the second emphasizes cellular chains and is appropriate for homology theory. We begin by constructing the various maps we need to define singular coface and codegeneracy operators.

Fix a positive integer $n$. For $0 \leqslant p \leqslant n$, let

$$
\underline{p}=\left\{\begin{array}{cc}
\varnothing, & p=0 \\
\{1, \ldots, p\}, & 1 \leqslant p \leqslant n
\end{array} \text { and } \bar{p}=\left\{\begin{array}{cc}
\varnothing, & p=0 \\
\{n-p+1, \ldots, n\}, & 1 \leqslant p \leqslant n
\end{array}\right.\right.
$$

then $\underline{p}$ and $\bar{p}$ contain the first and last $p$ elements of $\underline{n}$, respectively; note that $p \cap \bar{q}=\{p\}$ whenever $p+q=n+1$. Given integers $r, s \in \underline{n}$ such that $r+s=n+1$, there is a canonical projection $\Delta_{r, s}: P_{n} \rightarrow P_{r} \times P_{s}$ whose restriction to a vertex $v=a_{1}|\cdots| a_{n} \in P_{n}$ is given by

$$
\Delta_{r, s}(v)=b_{1}|\cdots| b_{r} \times c_{1}|\cdots| c_{s},
$$

where $\left(b_{1}, \ldots, b_{r} ; c_{1}, \ldots, c_{k-1}, c_{k+1}, \ldots, c_{s}\right)$ is the unshuffle of $\left(a_{1}, \ldots, a_{n}\right)$ with $b_{i} \in$ $\underline{r}, c_{j} \in \bar{s}, c_{k}=r$. For example, $\Delta_{2,3}(2|4| 1 \mid 3)=2|1 \times 2| 4 \mid 3$ and $\Delta_{3,2}(2|4| 1 \mid 3)=$ $2|1| 3 \times 4 \mid 3$. Since the image of the vertices of a cell of $P_{n}$ uniquely determines a cell in $P_{r} \times P_{s}$ the map $\Delta_{r, s}$ is well-defined and cellular. Furthermore, the restriction of $\Delta_{r, s}$ to an $(n-k)$-cell $A_{1}|\cdots| A_{k} \subset P_{n}$ is given by

$$
\Delta_{r, s}\left(A_{1}|\cdots| A_{k}\right)=\left\{\begin{array}{rr}
\underline{r} \times\left(A_{1}|\cdots| A_{i} \backslash \underline{r-1}|\cdots| A_{k}\right), & \text { if } \underline{r} \subseteq A_{i}, \text { some } i, \\
\left(A_{1}|\cdots| A_{j} \backslash \overline{s-1}|\cdots| A_{k}\right) \times \bar{s}, & \text { if } \bar{s} \subseteq A_{j}, \text { some } j, \\
\left(A_{1} \backslash \overline{s-1}|\cdots| A_{k} \backslash \overline{s-1}\right) & \\
\times\left(A_{1} \backslash \underline{r-1}|\cdots| A_{k} \backslash \underline{r-1}\right), & \text { otherwise. }
\end{array}\right.
$$

Note that $\Delta_{r, s}$ acts homeomorphically in the first two cases and degeneratively in the third when $1<k<n$. When $n=3$ for example, $\Delta_{2,2}$ maps the edge $1 \mid 23$ onto the edge $1 \mid 2 \times 23$ and the edge $13 \mid 2$ onto the vertex $1|2 \times 3| 2$ (see Figure 3 ). 


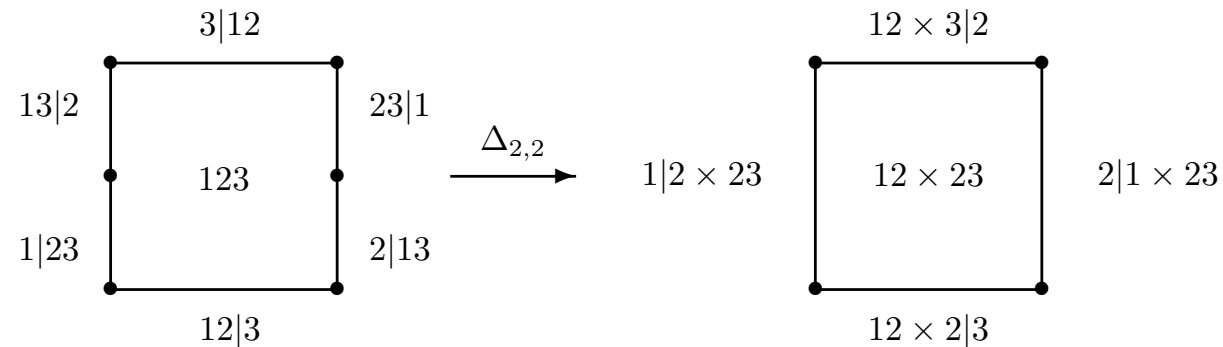

Figure 3: The projection $\Delta_{2,2}: P_{3} \rightarrow I^{2}$.
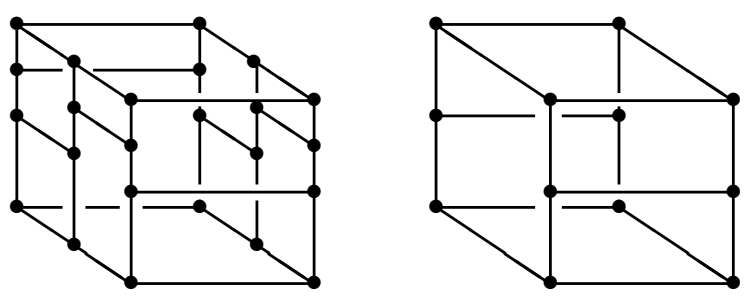

$\Delta_{2,3}$
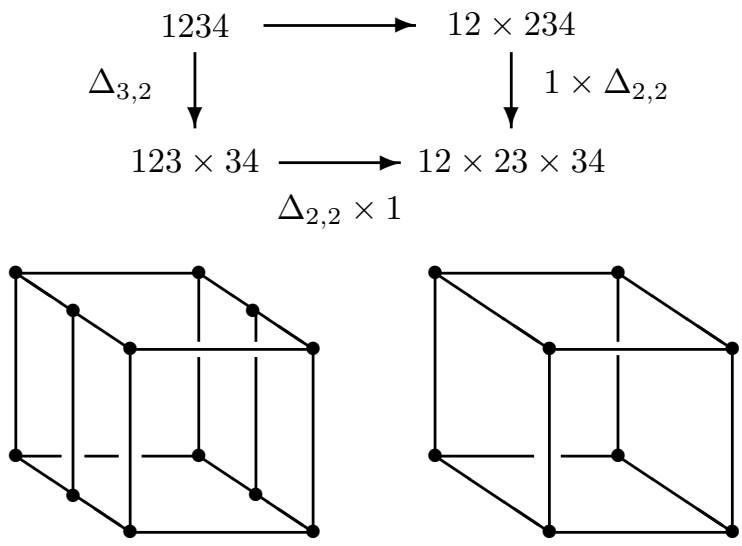

Figure 4: The projection $\rho_{4}: P_{4} \rightarrow I^{3}$.

Now identify the set $U=\left\{u_{1}<\cdots<u_{n}\right\}$ with $P_{n}$ and the ordered partitions of $U$ with the faces of $P_{n}$ in the obvious way. Then $\left(\Delta_{r, s} \times 1\right) \circ \Delta_{r+s-1, t}=\left(1 \times \Delta_{s, t}\right) \circ$ $\Delta_{r, s+t-1}$ whenever $r+s+t=n+2$ so that $\Delta_{*, *}$ acts coassociatively with respect to Cartesian product. It follows that each $k$-tuple $\left(n_{1}, \ldots, n_{k}\right) \in \mathbb{N}^{k}$ with $k \geqslant 2$ and $n_{1}+\cdots+n_{k}=n+k-1$ uniquely determines a cellular projection $\Delta_{n_{1} \cdots n_{k}}: P_{n} \rightarrow$ $P_{n_{1}} \times \cdots \times P_{n_{k}}$ given by the composition

$$
\Delta_{n_{1} \cdots n_{k}}=\left(\Delta_{n_{1}, n_{2}} \times 1^{\times k-2}\right) \circ \cdots \circ\left(\Delta_{n_{(k-2)}-k+3, n_{k-1}} \times 1\right) \circ \Delta_{n_{(k-1)}-k+2, n_{k}},
$$


where $n_{(q)}=n_{1}+\cdots+n_{q}$; and in particular,

$$
\Delta_{n_{1} \cdots n_{k}}(\underline{n})=\underline{n_{1}} \times \underline{n_{(2)}-1} \backslash \underline{n_{1}-1} \times \cdots \times \underline{n_{(k)}-(k-1)} \backslash \underline{n_{(k-1)}-(k-1)} .
$$

Note that formula 4.1 with $k=n-1$ and $n_{i}=2$ for all $i$ defines a projection $\rho_{n}: P_{n} \rightarrow I^{n-1}$

$$
\rho_{n}(\underline{n})=\Delta_{2 \cdots 2}(\underline{n})=12 \times 23 \times \cdots \times\{n-1, n\}
$$

(see Figure 4) acting on a vertex $u=u_{1}|\cdots| u_{n}$ as follows: For each $i \in \underline{n-1}$, let $\left\{u_{j}, u_{k} \mid j<k\right\}=\left\{u_{1}, \ldots, u_{n}\right\} \cap\{i, i+1\}$ and set $v_{i}=u_{j}, v_{i+1}=u_{k}$; then $\rho_{n}(u)=v_{1}\left|v_{2} \times \cdots \times v_{n-1}\right| v_{n}$.

Now choose a (non-cellular) homeomorphism $\gamma_{n}: I^{n-1} \rightarrow P_{n}$ whose restriction to a vertex $v=v_{1}\left|v_{2} \times \cdots \times v_{n-1}\right| v_{n}$ can be expressed inductively as follows: Set $A_{2}=v_{1} \mid v_{2}$; if $A_{k-1}$ has been obtained from $v_{1}\left|v_{2} \times \cdots \times v_{k-2}\right| v_{k-1}$, set

$$
A_{k}= \begin{cases}A_{k-1} \mid k, & \text { if } v_{k}=k \\ k \mid A_{k-1}, & \text { otherwise. }\end{cases}
$$

For example, $\gamma_{4}(2|1 \times 3| 2 \times 3 \mid 4)=3|2| 1 \mid 4$. Then $\gamma_{n}$ sends the vertices of $I^{n-1}$ to cubical vertices of $P_{n}$ and the vertices of $P_{n}$ fixed by $\gamma_{n} \rho_{n}$ are exactly its cubical vertices. Given a codimension 1 face $A \mid B \subset P_{n}$, index the elements of $A$ and $B$ as follows: If $n \in A$, write $A=\left\{a_{1}<\cdots<a_{m}\right\}$ and $B=\left\{b_{1}<\cdots<b_{\ell}\right\}$; if $n \in B$, write $A=\left\{a_{1}<\cdots<a_{\ell}\right\}$ and $B=\left\{b_{1}<\cdots<b_{m}\right\}$. Then $A \mid B$ uniquely embeds in $P_{n}$ as the subcomplex

$$
P_{\ell} \times P_{m}= \begin{cases}a_{1}|\cdots| a_{m}|B \times A| b_{1}|\cdots| b_{\ell}, & \text { if } n \in A \\ A\left|b_{1}\right| \cdots\left|b_{m} \times a_{1}\right| \cdots\left|a_{\ell}\right| B, & \text { if } n \in B .\end{cases}
$$

For example, $14 \mid 23$ embeds in $P_{4}$ as $1|4| 23 \times 14|2| 3$. Let $\iota_{A \mid B}: A \mid B \hookrightarrow P_{\ell} \times P_{m}$ denote this embedding and let $h_{A \mid B}=\iota_{A \mid B}^{-1}$; then $h_{A \mid B}: P_{\ell} \times P_{m} \rightarrow A \mid B$ is an orientation preserving homeomorphism. Also define the cellular projection

$$
\phi_{A \mid B}: P_{n} \rightarrow P_{\ell} \times P_{m}= \begin{cases}b_{1} \cdots b_{\ell} \times a_{1} \cdots a_{m}, & \text { if } n \in A \\ a_{1} \cdots a_{\ell} \times b_{1} \cdots b_{m}, & \text { if } n \in B\end{cases}
$$

on a vertex $c=c_{1}|\cdots| c_{n}$ by $\phi_{A \mid B}(c)=u_{1}|\cdots| u_{\ell} \times v_{1}|\cdots| v_{m}$, where $\left(u_{1}, \ldots, u_{\ell}\right.$; $\left.v_{1}, \ldots, v_{m}\right)$ is the unshuffle of $\left(c_{1}, \ldots, c_{n}\right)$ with $u_{i} \in B, v_{j} \in A$ when $n \in A$ or with $u_{i} \in A, v_{j} \in B$ when $n \in B$. Note that unlike $\Delta_{r, s}$, the projection $\phi_{A \mid B}$ always degenerates on the top cell; furthermore, $\phi_{A \mid B} \circ h_{A \mid B}=\phi_{B \mid A} \circ h_{A \mid B}=1$. We note that when $A$ or $B$ is a singleton set, the projection $\phi_{A \mid B}$ was defined by R.J. Milgram in [14].

The singular codegeneracy operator associated with $A \mid B$ is the map $\beta_{A \mid B}: P_{n} \rightarrow$ $P_{n-1}$ given by the composition

$$
P_{n} \stackrel{\phi_{A \mid B}}{\longrightarrow} P_{\ell} \times P_{m} \stackrel{\rho_{\ell} \times \rho_{m}}{\longrightarrow} I^{\ell-1} \times I^{m-1}=I^{n-2} \stackrel{\gamma_{n-1}}{\longrightarrow} P_{n-1} ;
$$

the singular coface operator associated with $A \mid B$ is the map $\delta_{A \mid B}: P_{n-1} \rightarrow P_{n}$ given by the composition

$$
P_{n-1} \stackrel{\rho_{n-1}}{\longrightarrow} I^{n-2}=I^{\ell-1} \times I^{m-1} \stackrel{\gamma_{\ell} \times \gamma_{m}}{\longrightarrow} P_{\ell} \times P_{m} \stackrel{h_{A \mid B}}{\longrightarrow} A \mid B \stackrel{i}{\hookrightarrow} P_{n} .
$$


Unlike the simplicial or cubical case, $\delta_{A \mid B}$ need not be injective. We shall often abuse notation and write $h_{A \mid B}: P_{\ell} \times P_{m} \rightarrow P_{n}$ when we mean $i \circ h_{A \mid B}$.

We are ready to define our first universal example. For future reference and to emphasize the fact that our definition depends only on positive integers, let $\left(n_{1}, \ldots, n_{k}\right) \in \mathbb{N}^{k}$ such that $n_{(k)}=n$ and denote

$$
\mathcal{P}_{n_{1} \cdots n_{k}}(n)=\left\{\text { Partitions } A_{1}|\cdots| A_{k} \text { of } \underline{n} \mid \# A_{i}=n_{i}\right\} \text {. }
$$

Definition 13. Let $Y$ be a topological space. The singular permutahedral set of $Y$ consists of the singular set

$$
\operatorname{Sing}_{*}^{P} Y=\bigcup_{n \geqslant 1}\left[\operatorname{Sing}_{n}^{P} Y=\left\{\text { Continuous maps } P_{n} \rightarrow Y\right\}\right]
$$

together with singular face and degeneracy operators

$$
d_{A \mid B}: \operatorname{Sing}_{n}^{P} Y \rightarrow \operatorname{Sing}_{n-1}^{P} Y \quad \text { and } \varrho_{A \mid B}: \operatorname{Sing}_{n-1}^{P} Y \rightarrow \operatorname{Sing}_{n}^{P} Y
$$

defined respectively for each $n \geqslant 2$ and $A \mid B \in \mathcal{P}_{* *}(n)$ as the pullback along $\delta_{A \mid B}$ and $\beta_{A \mid B}$, i.e., for $f \in \operatorname{Sing}_{n}^{P} Y$ and $g \in \operatorname{Sing}_{n-1}^{P} Y$,

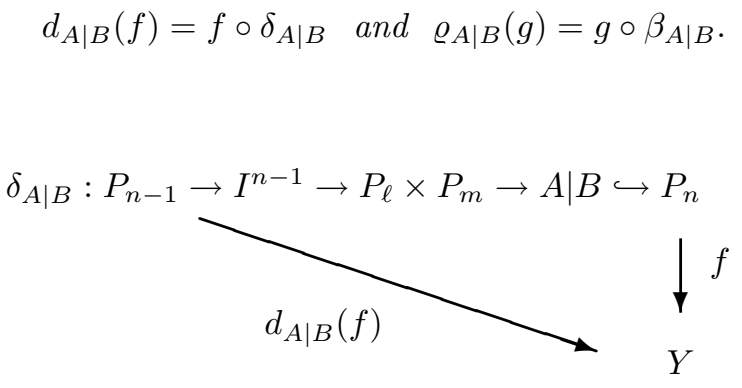

Figure 5: The singular face operator associated with $A \mid B$.

Although coface operators $\delta_{A \mid B}: P_{n-1} \rightarrow P_{n}$ need not be inclusions, the top cell of $P_{n-1}$ is always non-degenerate (c.f. Definition 20); however, the top cell of $P_{n-2}$ may degenerate under quadratic compositions $\delta_{A \mid B} \delta_{C \mid D}: P_{n-2} \rightarrow P_{n}$. For example, $\delta_{12 \mid 34} \delta_{13 \mid 2}: P_{2} \rightarrow P_{4}$ is a constant map, since $\delta_{12 \mid 34}: P_{3} \rightarrow P_{2} \times P_{2} \hookrightarrow P_{4}$ sends the edge $13 \mid 2$ to the vertex $1|2 \times 3| 2$.

Definition 14. A quadratic composition of face operators $d_{C \mid D} d_{A \mid B}$ acts on $P_{n}$ if the top cell of $P_{n-2}$ is non-degenerate under the composition

$$
\delta_{A \mid B} \delta_{C \mid D}: P_{n-2} \rightarrow P_{n} .
$$

Theorem 3 below gives the conditions under which a quadratic composition acts on $P_{n}$. For comparison, quadratic compositions of simplicial or cubical face operators always act on the simplex or cube. When $d_{C \mid D} d_{A \mid B}$ acts on $P_{n}$, we assign the label $d_{C \mid D} d_{A \mid B}$ to the codimension 2 face $\delta_{A \mid B} \delta_{C \mid D}(\underline{n})$. The various paths of descent from the top cell to a cell in codimension 2 gives rise to relations among compositions of face and degeneracy operators (see Figure 6). 


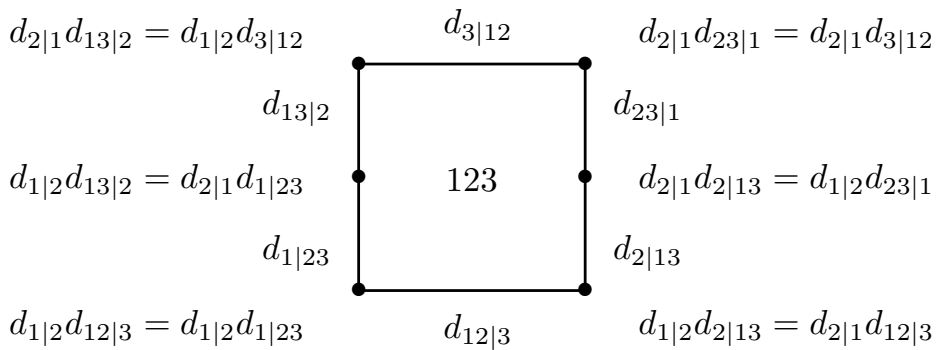

Figure 6: Quadratic relations on the vertices of $P_{3}$.

It is interesting to note that singular permutahedral sets have higher order structure relations, an example of which appears below in Figure 7 (see also (4.4)). This distinguishes permutahedral sets from simplicial or cubical sets in which relations are strictly quadratic. Our second universal example, called a "singular multipermutahedral set," specifies a singular permutahedral set by restricting to maps $f=\bar{f} \circ \Delta_{n_{1} \cdots n_{k}}$ for some continuous $\bar{f}: P_{n_{1}} \times \cdots \times P_{n_{k}} \rightarrow Y$. Face and degeneracy operators satisfy those relations above in which $\Delta_{n_{1} \cdots n_{k}}$ plays no essential role.

$\delta_{13 \mid 2}$

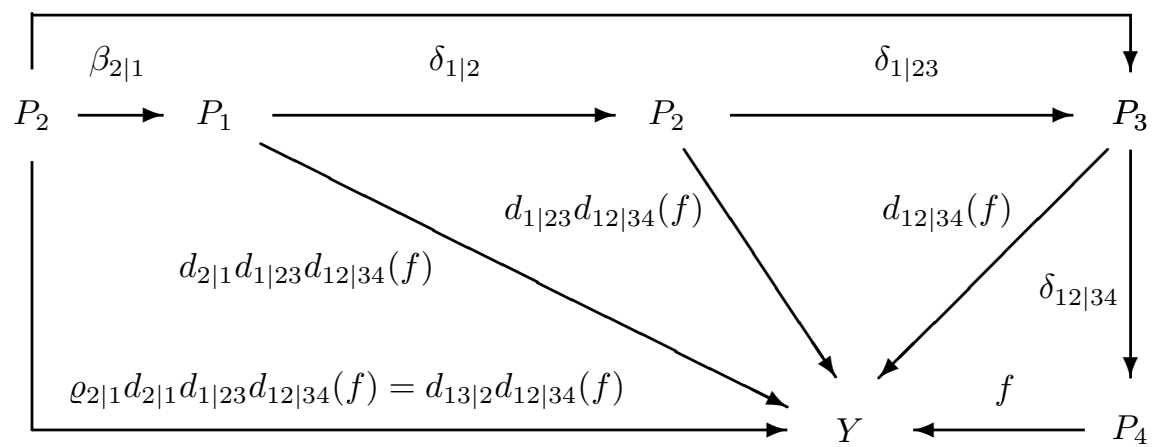

Figure 7: A quartic relation in $\operatorname{Sing}_{*}^{P} Y$.

Once again, fix a positive integer $n$, but this time consider $\left(n_{1}, \ldots, n_{k}\right) \in(\mathbb{N} \cup 0)^{k}$ with $n_{(k)}=n-1$ and the projection $\Delta_{n_{1}+1 \cdots n_{k}+1}: P_{n} \rightarrow P_{n_{1}+1} \times \cdots \times P_{n_{k}+1}$ with $\Delta_{n}: P_{n} \rightarrow P_{n}$ defined to be the identity. Given a topological space $Y$, let

$$
\operatorname{Sing}^{n_{1} \cdots n_{k}} Y=\left\{\bar{f} \circ \Delta_{n_{1}+1 \cdots n_{k}+1}: P_{n} \rightarrow Y \mid \bar{f} \text { is continuous }\right\} \text {; }
$$

define $f, f^{\prime} \in \operatorname{Sing}^{n_{1} \cdots n_{k}} Y$ to be equivalent if there exists $g: P_{n_{1}+1} \times \cdots \times P_{n_{i-1}+1} \times$ $P_{1} \times P_{n_{i+1}+1} \times \cdots \times P_{n_{k}+1} \rightarrow Y$ for some $i<k$ such that

$$
f=g \circ\left(1^{\times i-1} \times \phi_{\underline{n_{i}+1} \mid n_{i}+1} \times 1^{\times k-i-1}\right) \circ \Delta_{n_{1}+1 \cdots n_{i-1}+1, n_{i}+2, n_{i+2}+1 \cdots n_{k}+1}
$$


and

$$
f^{\prime}=g \circ\left(1^{\times i} \times \phi_{1 \mid \underline{n_{i+2}+1} \backslash 1} \times 1^{\times k-i-2}\right) \circ \Delta_{n_{1}+1 \cdots n_{i}+1, n_{i+2}+2, n_{i+3}+1 \cdots n_{k}+1},
$$

in which case we write $f \sim f^{\prime}$. The geometry of the cube motivates this equivalence; the degeneracies in the product of cubical sets implies the identification (c.f. [10] or the definition of the cubical set functor $\boldsymbol{\Omega} X$ in the Appendix).

Define the singular set

$$
\operatorname{Sing}_{n}^{M} Y=\bigcup_{\substack{\left(n_{1}, \ldots, n_{k}\right) \in(\mathbb{N} \cup 0)^{k} \\ n_{(k)}=n-1}} \operatorname{Sing}^{n_{1} \cdots n_{k}} Y / \sim .
$$

Singular face and degeneracy operators

$$
d_{A \mid B}: \operatorname{Sing}_{n}^{M} Y \rightarrow \operatorname{Sing}_{n-1}^{M} Y \text { and } \varrho_{A \mid B}: \operatorname{Sing}_{n-1}^{M} Y \rightarrow \operatorname{Sing}_{n}^{M} Y
$$

are defined piece-wise for each $n \geqslant 2$ and $A \mid B \in \mathcal{P}_{*, *}(n)$, depending on the form of $A \mid B$. More precisely, for each pair of integers $\left(p_{i}, q_{i}\right), 1 \leqslant i \leqslant k$, with

$$
\begin{gathered}
p_{i}=1+\sum_{j=1}^{i-1} n_{j} \text { and } q_{i}=1+\sum_{j=i+1}^{k} n_{j} \text {, let } \\
\mathcal{Q}_{p_{i}, q_{i}}(n)=\left\{U\left|V \in \mathcal{P}_{*, *}(n)\right|\left(\underline{p_{i}} \subseteq U \text { or } \underline{p_{i}} \subseteq V\right) \text { and }\left(\overline{q_{i}} \subseteq U \text { or } \overline{q_{i}} \subseteq V\right)\right\} ;
\end{gathered}
$$

in particular, when $r+s=n+1$, set $k=2, p_{1}=q_{2}=1, p_{2}=r$ and $q_{1}=s$, then

$$
\begin{gathered}
\mathcal{Q}_{r, 1}(n)=\left\{U\left|V \in \mathcal{P}_{*, *}(n)\right| \underline{r} \subseteq U \text { or } \underline{r} \subseteq V\right\} \text { and } \\
\mathcal{Q}_{1, s}(n)=\left\{U\left|V \in \mathcal{P}_{*, *}(n)\right| \bar{s} \subseteq U \text { or } \bar{s} \subseteq V\right\} .
\end{gathered}
$$

Since we identify $\underline{r} \mid \bar{s} \subset P_{n+1}$ with $P_{r} \times P_{s}=\Delta_{r, s}\left(P_{n}\right)$, it follows that $A \mid B \in$ $\mathcal{Q}_{p_{i}, q_{i}}(n)$ for some $i$ if and only if $\delta_{A \mid B} \delta_{\underline{r} \mid \bar{s}}: P_{n-1} \rightarrow P_{n+1}$ is non-degenerate; consequently we consider cases $A \mid B \in \mathcal{Q}_{p_{i}, q_{i}}(n)$ for some $i$ and $A \mid B \notin \mathcal{Q}_{p_{i}, q_{i}}(n)$ for all $i$.

Since our definitions of $d_{A \mid B}$ and $\varrho_{A \mid B}$ are independent in the first case and interdependent in the second, we define both operators simultaneously. But first we need some notation: Given an increasingly ordered set $M=\left\{m_{1}<\cdots<m_{k}\right\} \subset \mathbb{N}$, let $I_{M}: M \rightarrow \# M$ denote the indexing map $m_{i} \mapsto i$ and let $M+z=\left\{m_{i}+z\right\}$ denote translation by $\overline{z \in} \mathbb{Z}$. Of course, $M-z$ and $M+z$ are left and right translations when $z>0$; we adopt the convention that translation takes preference over set operations.

Assume $A \mid B \in \mathcal{Q}_{p_{i}, q_{i}}(n)$ for some $i$, and let

$$
\begin{gathered}
C_{i}=\left\{p_{i}, p_{i}+1, \ldots, p_{i}+n_{i}\right\} ; \\
A_{i}=\left(C_{i} \cap A\right)-n_{(i-1)}, \quad B_{i}=\left(C_{i} \cap B\right)-n_{(i-1)} ; \\
n_{i}^{\prime}=\#\left(A \cap C_{i}\right)-1, \quad n_{i}^{\prime \prime}=\#\left(B \cap C_{i}\right)-1 .
\end{gathered}
$$

For example, $n=6, n_{1}=3$ and $n_{2}=2$ determines the projection $\Delta_{4,3}: P_{6} \rightarrow$ $1234 \times 456$ and pairs $\left(p_{1}, q_{1}\right)=(1,3)$ and $\left(p_{2}, q_{2}\right)=(4,1)$. Thus $A|B=1234| 56 \in$ 
$\mathcal{Q}_{3,2}(6)$ and the composition $\delta_{\underline{4} \mid} \delta_{A \mid B}: P_{5} \rightarrow P_{7}$ is non-degenerate. Furthermore, $C_{2}=456, A_{2}=(456 \cap 1234)-3=1, B_{2}=23, n_{i}^{\prime}=0, n_{i}^{\prime \prime}=1$ and we may think of $d_{A \mid B}$ acting on $1234 \times 456$ as $1 \times d_{1 \mid 23}$.

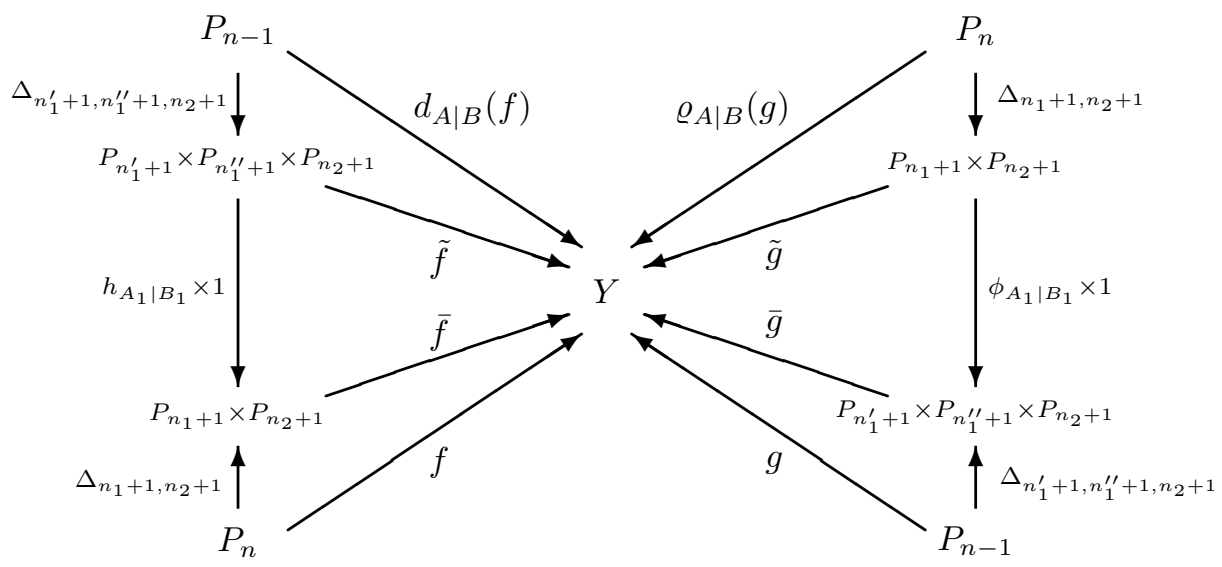

Figure 8: Face and degeneracy operators when $i=1$ and $k=2$.

For $f=\bar{f} \circ \Delta_{n_{1}+1 \cdots n_{k}+1} \in \operatorname{Sing}_{n}^{M} Y$, let $\tilde{f}=\bar{f} \circ\left(1^{\times i-1} \times h_{A_{i} \mid B_{i}} \times 1^{\times k-i}\right)$ and define

$$
d_{A \mid B}(f)=\tilde{f} \circ \Delta_{n_{1}+1 \cdots n_{i}^{\prime}+1, n_{i}^{\prime \prime}+1 \cdots n_{k}+1} .
$$

Dually, note that $n_{i}^{\prime}+n_{i}^{\prime \prime}=n_{i}-1$ implies the sum of coordinates $\left(n_{1}, \ldots, n_{i-1}, n_{i}^{\prime}, n_{i}^{\prime \prime}\right.$, $\left.n_{i+1}, \ldots, n_{k}\right) \in(\mathbb{N} \cup 0)^{k+1}$ is $n-2$. So for $g=\bar{g} \circ \Delta_{n_{1}+1 \cdots n_{i}^{\prime}+1, n_{i}^{\prime \prime}+1 \cdots n_{k}+1} \in$ $\operatorname{Sing}_{n-1}^{M} Y$, let $\tilde{g}=\bar{g} \circ\left(1^{\times i-1} \times \phi_{A_{i} \mid B_{i}} \times 1^{\times k-i}\right)$ and define

$$
\varrho_{A \mid B}(g)=\tilde{g} \circ \Delta_{n_{1}+1 \cdots n_{k}+1}
$$

(see Figure 8).

On the other hand, assume that $A \mid B \notin \mathcal{Q}_{p_{i}, q_{i}}(n)$ for all $i$ and define $d_{A \mid B}$ inductively as follows: When $k=2$, set $r=n_{1}+1, s=n_{2}+1$ and let

$$
\begin{aligned}
& K \mid L= \begin{cases}(\underline{r} \cap A) \cup \bar{s} \mid \underline{r} \cap B, \quad r \in A \\
\underline{r} \cap A \mid(\underline{r} \cap B) \cup \bar{s}, \quad r \in B\end{cases} \\
& M \mid N= \begin{cases}(\bar{s} \cap A)-1 \mid \underline{n-1} \backslash(\bar{s} \cap A)-1, & r \in B \\
\underline{n-1} \backslash(\bar{s} \cap B)-\# L \mid(\bar{s} \cap B)-\# L, & r \in A, \quad n \in A \\
I_{\underline{n} \backslash L}(A) \mid \underline{n-1} \backslash I_{\underline{n} \backslash L}(A), & r \in A, \quad n \in B\end{cases} \\
& C \mid D=\left\{\begin{array}{lll}
I_{\underline{n} \backslash B}(\underline{r} \cap A) \mid \underline{n-1} \backslash I_{\underline{n} \backslash B}(\underline{r} \cap A), & r \in B, & n \in B \\
I_{\underline{n} \backslash A}(\bar{s} \cap B) \mid \underline{n-1} \backslash I_{\underline{n} \backslash A}(\bar{s} \cap B), & r \in A, & n \in B \\
\underline{n-1} \backslash I_{\underline{n} \backslash B}(\bar{s} \cap A) \mid I_{\underline{n} \backslash B}(\bar{s} \cap A), & r \in B, & n \in A \\
\underline{n-1} \backslash I_{\underline{n} \backslash A}(\underline{r} \cap B) \mid I_{\underline{n} \backslash A}(\underline{r} \cap B), & r \in A, & n \in A .
\end{array}\right.
\end{aligned}
$$


Then define

$$
d_{A \mid B}=\varrho_{C \mid D} d_{M \mid N} d_{K \mid L} .
$$

Remark 1. This definition makes sense since $K\left|L \in \mathcal{Q}_{p_{1}, q_{1}}(n), M\right| N \in \mathcal{Q}_{p_{3}, q_{3}}(n-$ 1), $C \mid D \in \mathcal{Q}_{p_{1}, q_{1}}(n-1)$ with either $r, n \in B$ or $r, n \in A$ and $C \mid D \in \mathcal{Q}_{p_{3}, q_{3}}(n-1)$ with either $r \in B, n \in A$ or $r \in A, n \in B$. Of course, $\mathcal{Q}_{* *}(n-1)$ is considered with respect to the decomposition $n-2=m_{1}+m_{2}+m_{3}$ fixed after the action of $d_{K \mid L}(\underline{r} \times \underline{s})$.

If $k=3$, consider the pair $(r, s)=\left(n_{1}+1, n-n_{1}\right)$, then $\left(r_{1}, s_{1}\right)=\left(n_{2}+1, n-\right.$ $\left.n_{1}-n_{2}-1\right)$ for $A_{1}\left|B_{1}=I_{\underline{n} \backslash \underline{r}}(\bar{s} \cap A)\right| I_{\underline{n}} \backslash \underline{\underline{r}}(\bar{s} \cap B) \in \mathcal{P}_{p_{1}, q_{1}}(n-r)$, and so on. Now dualize and use the same formulas above to define the degeneracy operator $\varrho_{A \mid B}$.

Definition 15. Let $Y$ be a topological space. The singular multipermutahedral set of $Y$ consists of the singular set $\operatorname{Sing}_{*}^{M} Y$ together with the singular face and degeneracy operators

$$
d_{A \mid B}: \operatorname{Sing}_{n}^{M} Y \rightarrow \operatorname{Sing}_{n-1}^{M} Y \quad \text { and } \varrho_{A \mid B}: \operatorname{Sing}_{n-1}^{M} Y \rightarrow \operatorname{Sing}_{n}^{M} Y
$$

defined respectively for each $n \geqslant 2$ and $A \mid B \in \mathcal{P}_{* *}(n)$.

Remark 2. The operator $d_{A \mid B}$ defined in (4.3) applied to $d_{U \mid V}$ for some $U \mid V \in$ $\mathcal{P}_{r, s}(n+1)$ yields the higher order structural relation

$$
d_{A \mid B} d_{U \mid V}=\varrho_{C \mid D} d_{M \mid N} d_{K \mid L} d_{U \mid V}
$$

discussed in our first universal example.

Now $\operatorname{Sing}_{*}^{M} Y$ determines the singular (co)homology of a space $Y$ in the following way: Let $R$ be a commutative ring with identity. For $n \geqslant 1$, let $C_{n-1}\left(\operatorname{Sing}^{M} Y\right)$ denote the $R$-module generated by $\operatorname{Sing}_{n}^{M} Y$ and form the "chain complex"

$$
\left(C_{*}\left(\operatorname{Sing}^{M} Y\right), d\right)=\bigoplus_{\substack{n_{(k)}=n-1 \\ n \geqslant 1}}\left(C_{n-1}\left(\operatorname{Sing}^{n_{1} \cdots n_{k}} Y\right), d_{n_{1} \cdots n_{k}}\right),
$$

where

$$
d_{n_{1} \cdots n_{k}}=\sum_{A \mid B \in \bigcup_{i=1}^{k} \mathcal{Q}_{p_{i}, q_{i}}(n)}-(-1)^{n_{(i-1)}+n_{i}^{\prime}} \quad \operatorname{shuff}\left(C_{i} \cap A ; C_{i} \cap B\right) d_{A \mid B} .
$$

Refer to the example in Figure 7 and note that for $f \in C_{4}\left(\operatorname{Sing}^{M} Y\right)$ with $d_{13 \mid 2} d_{12 \mid 34}(f) \neq 0$, the component $d_{13 \mid 2} d_{12 \mid 34}(f)$ of $d^{2}(f) \in C_{2}\left(\operatorname{Sing}^{M} Y\right)$ is not cancelled and $d^{2} \neq 0$. Hence $d$ is not a differential. To remedy this, form the quotient

$$
C_{*}^{\diamond}(Y)=C_{*}\left(\operatorname{Sing}^{M} Y\right) / D G N
$$

where $D G N$ is the submodule generated by the degeneracies, and obtain the singular permutahedral chain complex $\left(C_{*}^{\diamond}(Y), d\right)$. Because the signs in $d$ are determined by the index $i$, which is missing in our first universal example, we are unable to use our first example to define a chain complex with signs. However, we could use it to define a unoriented theory with $\mathbb{Z}_{2}$-coefficients. 
The singular homology of $Y$ is recovered from the composition

$$
C_{*}(\operatorname{Sing} Y) \rightarrow C_{*}\left(\operatorname{Sing}^{I} Y\right) \rightarrow C_{*}\left(\operatorname{Sing}^{M} Y\right) \rightarrow C_{*}^{\diamond}(Y)
$$

arising from the canonical cellular projections

$$
P_{n+1} \rightarrow I^{n} \rightarrow \Delta^{n}
$$

Since this composition is a chain map, there is a natural isomorphism

$$
H_{*}(Y) \approx H_{*}^{\diamond}(Y)=H_{*}\left(C_{*}^{\diamond}(Y), d\right) .
$$

The fact that our diagonal on $P$ and the A-W diagonal on simplices commute with projections allows us to recover the singular cohomology ring of $Y$ as well. Finally, we remark that a cellular projection $f$ between polytopes induces a chain map between corresponding singular chain complexes whenever chains on the target are normalized. Here $C_{*}(\operatorname{Sing} Y)$ and $C_{*}\left(\operatorname{Sing}^{I} Y\right)$ are non-normalized and the induced map $f^{*}$ is not a chain map; but fortunately $d^{2}=0$ does not depend $d f^{*}=f^{*} d$.

\subsection{Abstract Permutahedral Sets}

We begin by constructing a generating category $\mathbf{P}$ for permutahedral sets similar to that of finite ordered sets and monotonic maps for simplicial sets. The objects of $\mathbf{P}$ are the sets $n !=S_{n}$ of permutations of $\underline{n}, n \geqslant 1$. But before we can define the morphisms we need some preliminaries. First note that when $P_{n}$ is identified with its vertices $n$ !, the maps $\rho_{n}$ and $\gamma_{n}$ defined above become

$$
\rho_{n}: n ! \rightarrow 2 !^{n-1} \text { and } \gamma_{n}: 2 !^{n-1} \rightarrow n !
$$

Given a non-empty increasingly ordered set $M=\left\{m_{1}<\cdots<m_{k}\right\} \subset \mathbb{N}$, let $M$ ! denote the set of all permutations of $M$ and let $J_{M}: M ! \rightarrow k$ ! be the map defined for $a=\left(m_{\sigma(1)}, \ldots, m_{\sigma(k)}\right) \in M$ ! by $J_{M}(a)=\sigma$. For $n, m \in \mathbb{N}$ and partitions $A_{1}|\cdots| A_{k} \in \mathcal{P}_{n_{1} \cdots n_{k}}(n)$ and $B_{1}|\cdots| B_{\ell} \in \mathcal{P}_{m_{1} \cdots m_{\ell}}(m)$ with $n-k=m-\ell=\varkappa$, define the morphism

$$
f_{A_{1}|\cdots| A_{k}}^{B_{1}|\cdots| B_{\ell}}: m ! \rightarrow n !
$$

by the composition

$m ! \stackrel{s h_{B}}{\rightarrow} \prod_{j=1}^{\ell} B_{j} \stackrel{\sigma_{\max }}{\longrightarrow} \prod_{r=1}^{\ell} B_{j_{r}} \stackrel{J_{B}}{\rightarrow} \prod_{j=r}^{\ell} m_{j_{r}} ! \stackrel{\rho_{*}}{\rightarrow} 2 !^{\varkappa} \stackrel{\gamma_{*}}{\rightarrow} \prod_{s=1}^{k} n_{i_{s}} ! \stackrel{J_{A}^{-1}}{\rightarrow} \prod_{s=1}^{k} A_{i_{s}} \stackrel{\sigma_{\text {max }}^{-1}}{\rightarrow} \prod_{i=1}^{k} A_{i} \stackrel{\iota_{A}}{\rightarrow} n !$

where $s h_{B}$ is a surjection defined for $b=\left\{b_{1}, \ldots, b_{m}\right\} \in m$ ! by

$$
s h_{B}(b)=\left(b_{1,1}, . ., b_{m_{1}, 1} ; \ldots ; b_{1, \ell}, . ., b_{m_{\ell}, \ell}\right),
$$

in which the right-hand side is the unshuffle of $b$ with $b_{r, t} \in B_{t}, 1 \leqslant r \leqslant m_{t}, 1 \leqslant$ $t \leqslant \ell ; \sigma_{\max } \in S_{\ell}$ is a permutation defined by $j_{r}=\sigma_{\max }(r), \max B_{j_{r}}=\max \left(B_{1} \cup\right.$ $\left.B_{2} \cup \cdots \cup B_{j_{r}}\right) ; J_{B}=\prod_{r=1}^{\ell} J_{B_{j_{r}}} ; \rho_{*}=\prod_{r=1}^{\ell} \rho_{j_{r}}$ and $\gamma_{*}=\prod_{s=1}^{k} \gamma_{i_{s}}$; finally, $\iota_{A}$ is the inclusion. It is easy to see that

$$
f_{A_{1}|\cdots| A_{k}}^{B_{1}|\cdots| B_{\ell}}=f \frac{\varkappa+1}{A_{1}|\cdots| A_{k}} \circ f_{\underline{\varkappa+1}}^{B_{1}|\cdots| B_{\ell}} \text { and } f_{\underline{n}}^{\frac{n}{n}}=\gamma_{n} \circ \rho_{n} .
$$


In particular, the maps $f \frac{n-1}{A \mid B}:(n-1) ! \rightarrow n !$ and $f_{\underline{n-1}}^{A \mid B}: n ! \rightarrow(n-1) !$ are generator morphisms denoted by $\delta_{A \mid B}$ and $\beta_{A \mid B}$, respectively (see Theorem 2 below, the statement of which requires some new set operations).

Definition 16. Given non-empty disjoint subsets $A, B, U \subset \underline{n+1}$ with $A \cup B \subseteq U$, define the lower and upper disjoint unions (with respect to $U \overline{\text { ) by }}$

and

$$
A \underline{\sqcup} B= \begin{cases}I_{U \backslash A}(B)+\# A-1, & \text { if } \min B>\min (U \backslash A) \\ I_{U \backslash A}(B)+\# A-1 \cup \underline{\# A}, & \text { if } \min B=\min (U \backslash A)\end{cases}
$$

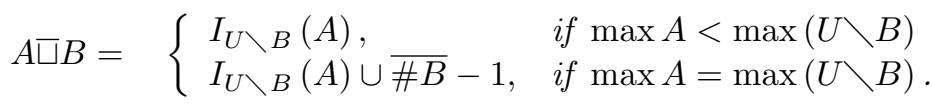

If either $A$ or $B$ is empty, define $A \sqcup B=A \square B=A \cup B$. Furthermore, given nonempty disjoint subsets $A, B_{1}, \ldots, B_{k} \subset \underline{n+1}$ with $k \geqslant 1$, set $U=A \cup B_{1} \cup \cdots \cup B_{k}$ and define

$$
A \square\left(B_{1}|\cdots| B_{k}\right)=\left(B_{1}|\cdots| B_{k}\right) \square A= \begin{cases}A \sqsubseteq B_{1}|\cdots| A \sqsubseteq B_{k}, & \text { if } \max A<\max U \\ B_{1} \bar{\square} A|\cdots| B_{k} \bar{\square} A, & \text { if } \max A=\max U .\end{cases}
$$

Note that if $A \mid B$ is a partition of $\underline{n+1}$, then

$$
A \underline{\sqcup} B=A \bar{\square} B=\underline{n} .
$$

Given a partition $A_{1}|\cdots| A_{k+1}$ of $\underline{n}$, define $A_{1}^{1}|\cdots| A_{k+1}^{1}=A_{1}^{1}|\cdots| A_{1}^{k+1}=$ $A_{1}|\cdots| A_{k+1}$; inductively, given $A_{1}^{i}|\cdots| A_{k-i+2}^{i}$ the partition of $\underline{n-i+1}, 1 \leqslant i<k$, let

$$
A_{1}^{i+1}|\cdots| A_{k-i+1}^{i+1}=A_{1}^{i} \square\left(A_{2}^{i}|\cdots| A_{k-i+2}^{i}\right)
$$

be the partition of $\underline{n-i}$; and given $A_{i}^{1}|\cdots| A_{i}^{k-i+2}$ the partition of $\underline{n-i+1}, 1 \leqslant$ $i<k$, let

$$
A_{i+1}^{1}|\cdots| A_{i+1}^{k-i+1}=\left(A_{i}^{1}|\cdots| A_{i}^{k-i+1}\right) \square A_{i}^{k-i+2}
$$

be the partition of $\underline{n-i}$.

Theorem 2. For $A_{1}|\cdots| A_{k+1} \in \mathcal{P}_{n_{1} \cdots n_{k+1}}(n), 2 \leqslant k \leqslant n$, the map $f \frac{n-k}{A_{1}|\cdots| A_{k+1}}$ : $(n-k) ! \rightarrow n !$ can be expressed as a composition of $\delta$ 's two ways:

$$
f \frac{n-k}{A_{1}|\cdots| A_{k+1}}=\delta_{A_{1}^{1} \mid A_{2}^{1} \cup \cdots \cup A_{k+1}^{1}} \cdots \delta_{A_{1}^{k} \mid A_{2}^{k}}=\delta_{A_{1}^{1} \cup \cdots \cup A_{1}^{k} \mid A_{1}^{k+1}} \cdots \delta_{A_{k}^{1} \mid A_{k}^{2}} .
$$

Proof. The proof is straightforward and omitted.

There is also the dual set of relations among the $\beta$ 's.

Example 5. Theorem 2 defines structure relations among the $\delta$ 's, the first of which is

$$
\delta_{A \mid B \cup C} \delta_{A \square(B \mid C)}=\delta_{A \cup B \mid C} \delta_{(A \mid B) \square C}
$$

when $k=2$. In particular, let $A|B| C=12|345| 678$. Since $A \sqsubseteq B=\{1234\}, A \unrhd C=$ $\{567\}, A \square C=\{12\}$ and $B \bar{\square} C=\{34567\}$, we obtain the following quadratic relation on $12|345| 678$ :

$$
\delta_{12 \mid 345678} \delta_{1234 \mid 567}=\delta_{12345 \mid 678} \delta_{12 \mid 34567}
$$


similarly, on $345|12| 678$ we have

$$
\delta_{345 \mid 12678} \delta_{1234 \mid 567}=\delta_{12345 \mid 678} \delta_{34567 \mid 12} .
$$

Theorem 3. Let $A \mid B \in \mathcal{P}_{p, q}(n+1)$ and $C \mid D \in \mathcal{P}_{* *}(n)$. Then $\delta_{A \mid B} \delta_{C \mid D}$ coincides with a map $f \frac{n-1}{X|Y| Z}:(n-1) ! \rightarrow(n+1)$ ! if and only if

$$
C \mid D \in \begin{cases}\mathcal{Q}_{q, 1}(n) \cup \mathcal{Q}_{1, p}(n), & \text { if } n+1 \in A \\ \mathcal{Q}_{p, 1}(n) \cup \mathcal{Q}_{1, q}(n), & \text { if } n+1 \in B .\end{cases}
$$

Proof. If $\delta_{A \mid B} \delta_{C \mid D}$ coincides with $f_{X|Y| Z}^{n-1}$, then according to relation (4.7) we have either

$$
A|B=X| Y \cup Z \text { and } C \mid D=X \square(Y \mid Z)
$$

or

$$
A|B=X \cup Y| Z \text { and } C \mid D=(X \mid Y) \square Z .
$$

Hence there are two cases.

Case 1: $A|B=X| Y \cup Z$.

Subcase 1a: Assume $n+1 \in A$. If $\max Y=\max (Y \cup Z)$, then $\bar{p} \subseteq Y \square X$; otherwise

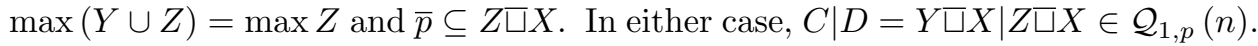

Subcase 1b: Assume $n+1 \in B$. If $\min Y=\min (Y \cup Z)$, then $p \subseteq X \sqsubseteq Y$; otherwise $\min (Y \cup Z)=\min Z$ and $\underline{p} \subseteq X \sqsubseteq Z$. In either case, $C|D=X \sqsubseteq \bar{Y}| X \sqsubseteq Z \in \mathcal{Q}_{p, 1}(n)$.

Case 2: $A|B=X \cup Y| Z$.

Subcase 2a: Assume $n+1 \in A$. If $\min X=\min (X \cup Y)$, then $q \subseteq Z \sqcup X$; otherwise $\min (X \cup Y)=\min Y$ and $q \subseteq Z \sqsubseteq Y$. In either case, $C|D=Z \sqsubseteq \bar{X}| Z \sqsubseteq Y \in \mathcal{Q}_{q, 1}(n)$.

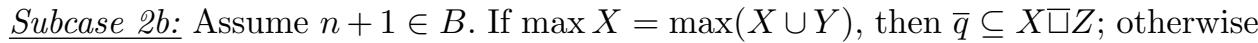
$\max (X \cup Y)=\max Y$ and $\bar{q} \subseteq Y \square Z$. In either case, $C|D=X \square Z| Y \square Z \in \mathcal{Q}_{1, q}(n)$.

Conversely, given $A \mid B \in \mathcal{P}_{p, q}(n+1)$ and $C \mid D$ satisfying conditions (4.6) above, let

$$
[A|B ; C| D]= \begin{cases}A\left|I_{B}^{-1}(\bar{q} \cap C-p+1)\right| I_{B}^{-1}(\bar{q} \cap D-p+1), & C \mid D \in \mathcal{Q}_{p, 1}(n) \\ I_{A}^{-1}(\underline{p} \cap C)\left|I_{A}^{-1}(\underline{p} \cap D)\right| B, & C \mid D \in \mathcal{Q}_{1, q}(n)\end{cases}
$$

and

$$
[A|B ; C| D]= \begin{cases}A\left|I_{B}^{-1}(\underline{q} \cap C)\right| I_{B}^{-1}(\underline{q} \cap D), & C \mid D \in \mathcal{Q}_{1, p}(n) \\ I_{A}^{-1}(\bar{p} \cap C-q+1)\left|I_{A}^{-1}(\bar{p} \cap D-q+1)\right| B, & C \mid D \in \mathcal{Q}_{q, 1}(n) .\end{cases}
$$

A straightforward calculation shows that

$$
[X \mid Y \cup Z ; X \square(Y \mid Z)]=X|Y| Z=[X \cup Y \mid Z ; \quad(X \mid Y) \square Z] .
$$

Consequently, if $X|Y| Z=[A|B ; C| D]$, either

$$
A|B=X| Y \cup Z \text { and } C \mid D=X \square(Y \mid Z)
$$


when $C \mid D \in \mathcal{Q}_{p, 1}(n) \cup \mathcal{Q}_{1, p}(n)$ or

$$
A|B=X \cup Y| Z \text { and } C \mid D=(X \mid Y) \square Z
$$

when $C \mid D \in \mathcal{Q}_{q, 1}(n) \cup \mathcal{Q}_{1, q}(n)$.

On the other hand, if $C \mid D \notin \mathcal{Q}_{p, 1}(n) \cup \mathcal{Q}_{1, p}(n) \cup \mathcal{Q}_{q, 1}(n) \cup \mathcal{Q}_{1, q}(n)$, higher order structure relations involving both coface and codegeneracy operators appear.

Definition 17. Let $\mathcal{C}$ be the category of sets. A permutahedral set is a contravariant functor

$$
\mathcal{Z}: \mathbf{P} \rightarrow \mathcal{C}
$$

Thus a permutahedral set $\mathcal{Z}$ is a graded set $\mathcal{Z}=\left\{\mathcal{Z}_{n}\right\}_{n \geqslant 1}$ endowed with face and degeneracy operators

$$
d_{A \mid B}=\mathcal{Z}\left(\delta_{A \mid B}\right): \mathcal{Z}_{n} \rightarrow \mathcal{Z}_{n-1} \text { and } \varrho_{M \mid N}=\mathcal{Z}\left(\beta_{M \mid N}\right): \mathcal{Z}_{n} \rightarrow \mathcal{Z}_{n+1}
$$

satisfying an appropriate set of relations, which includes quadratic relations such as

$$
d_{A \square(B \mid C)} d_{A \mid B \cup C}=d_{(A \mid B) \square C} d_{A \cup B \mid C}
$$

induced by (4.5) and higher order relations such as

$$
d_{A \mid B} d_{U \mid V}=\varrho_{C \mid D} d_{M \mid N} d_{K \mid L} d_{U \mid V}
$$

discussed in (4.4).

Let us define the abstract analog of a singular multipermutahedral set, which leads to a singular chain complex with arbitrary coefficients.

Definition 18. For $n \geqslant 1$, let $X_{n}=\bigcup_{n_{(k)}=n-1, n_{k} \geqslant 0} X^{n_{1} \cdots n_{k}}$ and $X_{n-1}=$ $\bigcup_{m_{(\ell)}=n-2, m_{\ell} \geqslant 0} X^{m_{1} \cdots m_{\ell}}$ be filtered sets; let $A \mid B \in \mathcal{Q}_{p_{i}, q_{i}}(n)$ for some $i$. A map $g: X_{n} \rightarrow X_{n-1}$ acts as an $A \mid B$-formal derivation if

$$
\left.g\right|_{X^{n_{1} \cdots n_{k}}: X^{n_{1} \cdots n_{k}}} \rightarrow X^{n_{1} \cdots n_{i}^{\prime}, n_{i}^{\prime \prime} \cdots n_{k}}
$$

where $\left(n_{i}^{\prime}, n_{i}^{\prime \prime}\right)$ is given by (4.2).

Let $\mathcal{C}_{M}$ denote the category whose objects are positively graded sets $X_{*}$ filtered by subsets $X_{n}=\bigcup_{n_{(k)}=n-1, n_{k} \geqslant 0} X^{n_{1} \cdots n_{k}}$ and whose morphisms are filtration preserving set maps.

Definition 19. A multipermutahedral set is a contravariant functor $\mathcal{Z}: \mathbf{P} \rightarrow \mathcal{C}_{M}$ such that

$$
\mathcal{Z}\left(\delta_{A \mid B}\right): \mathcal{Z}(n !) \rightarrow \mathcal{Z}((n-1) !)
$$

acts as an $A \mid B$-formal derivation for each $A \mid B \in \mathcal{Q}_{p_{i}, q_{i}}$, all $i \geqslant 1$.

Thus a multipermutahedral set $\mathcal{Z}$ is a graded set $\left\{\mathcal{Z}_{n}\right\}_{n \geqslant 1}$ with

$$
\mathcal{Z}_{n}=\bigcup_{\substack{n_{(k)}=n-1 \\ n_{k} \geqslant 0}} \mathcal{Z}^{n_{1} \cdots n_{k}},
$$


together with face and degeneracy operators

$$
d_{A \mid B}=\mathcal{Z}\left(\delta_{A \mid B}\right): \mathcal{Z}_{n} \rightarrow \mathcal{Z}_{n-1} \text { and } \varrho_{M \mid N}=\mathcal{Z}\left(\beta_{M \mid N}\right): \mathcal{Z}_{n} \rightarrow \mathcal{Z}_{n+1}
$$

satisfying the relations of a permutahedral set and the additional requirement that $d_{A \mid B}$ respect underlying multigrading. This later condition allows us to form the chain complex of $\mathcal{Z}$ with signs mimicking the cellular chain complex of permutahedra (see below). Note that the chain complex of a permutahedral set is only defined with $\mathbb{Z}_{2}$-coefficients in general.

\subsection{The Cartesian product of permutahedral sets}

The objects and morphisms in the category $\mathbf{P} \times \mathbf{P}$ are the sets and maps

$$
n ! !=\bigcup_{r+s=n} r ! \times s ! \text { and } \bigcup_{f, g \in \mathbf{P}} f \times g: m ! ! \rightarrow n ! !
$$

all $m, n \geqslant 1$. There is a functor $\Delta: \mathbf{P} \rightarrow \mathbf{P} \times \mathbf{P}$ defined as follows. If $A \mid B \in \mathcal{Q}_{r, 1}(n) \cup$ $\mathcal{Q}_{1, s}(n)$, define $\Delta_{r, s}(A \mid B)=A_{1}\left|B_{1} \times A_{2}\right| B_{2} \in r ! \times s !$ and define $\delta_{A \mid B}:(n-1) ! \rightarrow n !$ by

$$
\Delta\left(\delta_{A \mid B}\right)=\delta_{A_{1} \mid B_{1}} \times \delta_{A_{2} \mid B_{2}},
$$

where $\delta_{A_{i} \mid B_{i}}=1$ for either $i=1$ or $i=2$. Define $\Delta\left(\beta_{A \mid B}\right)$ similarly. On the other hand, if $A \mid B \notin \mathcal{Q}_{r, 1}(n) \cup \mathcal{Q}_{1, s}(n)$, define

$$
\Delta\left(\delta_{A \mid B}\right)=\Delta\left(\delta_{K \mid L}\right) \Delta\left(\delta_{M \mid N}\right) \Delta\left(\beta_{C \mid D}\right),
$$

where $K|L, M| N, C \mid D$ are given by the formulas in (4.3). Dually, define $\Delta\left(\beta_{M \mid N}\right)$. It is easy to check that $\Delta$ is well defined.

Given multipermutahedral sets $\mathcal{Z}^{\prime}, \mathcal{Z}^{\prime \prime}: \mathbf{P} \rightarrow \mathcal{C}_{M}$, first define a functor

$$
\mathcal{Z}^{\prime} \tilde{\times} \mathcal{Z}^{\prime \prime}: \mathbf{P} \times \mathbf{P} \rightarrow \mathcal{C}_{M}
$$

on an object $n !$ ! by

$$
\left(\mathcal{Z}^{\prime} \tilde{\times} \mathcal{Z}^{\prime \prime}\right)(n ! !)=\bigcup_{r+s=n} \mathcal{Z}^{\prime}(r !) \times \mathcal{Z}^{\prime \prime}(s !) / \sim,
$$

where $(a, b) \sim(c, e)$ if and only if $a=\varrho_{\underline{r} \mid r+1}^{\prime}(c)$ and $e=\varrho_{1 \mid \underline{s+1} \backslash \underline{1}}^{\prime \prime}(b)$. On a map $h=\bigcup(f \times g): m ! ! \rightarrow n ! !$,

$$
\left(\mathcal{Z}^{\prime} \tilde{\times} \mathcal{Z}^{\prime \prime}\right)(h):\left(\mathcal{Z}^{\prime} \tilde{\times} \mathcal{Z}^{\prime \prime}\right)(n ! !) \rightarrow\left(\mathcal{Z}^{\prime} \tilde{\times} \mathcal{Z}^{\prime \prime}\right)(m ! !)
$$

is the map induced by $\bigcup\left(\mathcal{Z}^{\prime}(f) \times \mathcal{Z}^{\prime \prime}(g)\right)$. Now define the product $\mathcal{Z}^{\prime} \times \mathcal{Z}^{\prime \prime}$ to be the composition of functors

$$
\mathcal{Z}^{\prime} \times \mathcal{Z}^{\prime \prime}=\mathcal{Z}^{\prime} \tilde{\times} \mathcal{Z}^{\prime \prime} \circ \Delta: \mathbf{P} \rightarrow \mathcal{C}_{M}
$$

The face operator $d_{A \mid B}$ on $\mathcal{Z}^{\prime} \times \mathcal{Z}^{\prime \prime}$ is given by

$$
d_{A \mid B}(a \times b)= \begin{cases}d_{\underline{r} \cap A \mid \underline{r} \cap B}^{\prime}(a) \times b, & \text { if } A \mid B \in \mathcal{Q}_{1, s}(n), \\ a \times d_{(\bar{s} \cap A)-r+1 \mid(\bar{s} \cap B)-r+1}^{\prime \prime}(b), & \text { if } A \mid B \in \mathcal{Q}_{r, 1}(n), \\ \varrho_{C \mid D} d_{M \mid N} d_{K \mid L}(a \times b), & \text { otherwise, }\end{cases}
$$

with $M|N, K| L, C \mid D$ given by the formulas in (4.3). 
Example 6. The canonical map $\iota: \operatorname{Sing}^{P} X \times \operatorname{Sing}^{P} Y \rightarrow \operatorname{Sing}^{P}(X \times Y)$ defined for $(f, g) \in \operatorname{Sing}_{r}^{P} X \times \operatorname{Sing}_{s}^{P} Y$ by

$$
\iota(f, g)=(f \times g) \circ \Delta_{r, s}
$$

is a map of permutahedral sets. Consequently, if $X$ is a topological monoid, the singular permutahedral complex $\operatorname{Sing}^{P} X$ inherits a canonical monoidal structure.

\subsection{The diagonal on a permutahedral set}

Let $\mathcal{Z}=\left(\mathcal{Z}_{n}, d_{A \mid B}, \varrho_{M \mid N}\right)$ be a multipermutahedral set. The chain complex of $\mathcal{Z}$ is

$$
C_{*}^{\diamond}(\mathcal{Z})=C_{*}(\mathcal{Z}) / D G N
$$

where $D G N$ is the submodule generated by the degeneracies;

$$
\left(C_{*}(\mathcal{Z}), d\right)=\bigoplus_{\substack{n(k)+1=n \\ n \geqslant 1}}\left(C_{n-1}\left(\mathcal{Z}^{n_{1} \cdots n_{k}}\right), d_{n_{1} \cdots n_{k}}\right)
$$

and

$$
d_{n_{1} \cdots n_{k}}=\sum_{A \mid B \in \bigcup_{i=1}^{k} \mathcal{Q}_{p_{i}, q_{i}}(n)}-(-1)^{n_{(i-1)}+n_{i}^{\prime}} \operatorname{shuff}\left(C_{i} \cap A ; C_{i} \cap B\right) d_{A \mid B} .
$$

The explicit diagonal

$$
\Delta: C_{*}^{\diamond}(\mathcal{Z}) \rightarrow C_{*}^{\diamond}(\mathcal{Z}) \otimes C_{*}^{\diamond}(\mathcal{Z})
$$

on $a \subset \mathcal{Z}_{n}$ is given by

$$
\Delta(a)=\sum_{\substack{F \in \mathcal{C}^{q \times n-q+2} \\ 0 \leqslant q \leqslant n}} \operatorname{csgn}(F) d_{c(F)}(a) \otimes d_{r(F)}(a),
$$

where $d_{A_{1}|\ldots| A_{k+1}}=\mathcal{Z}\left(f \frac{n-k}{A_{1}|\ldots| A_{k+1}}\right)$.

\subsection{The double cobar-construction $\Omega^{2} C_{*}(X)$}

Given a simplicial, cubical or a permutahedral set $W$ with base point *, let $C_{*}(W)$ denote the

quotient $C_{*}(W) / C_{>0}(*)$. Say that $W$ is $k$-reduced if $W_{i}$ contains exactly one element for each $i \leqslant k$ and let $\Omega C$ denote the cobar construction on a 1-reduced DG coalgebra C. In [8] and [9], Kadeishvili and Saneblidze construct functors from the category of 1-reduced simplicial sets to the category of cubical sets and from the category of 1-reduced cubical sets to the category of multipermutahedral sets (denoted by $\Omega$ in either case) for which the following statements hold (c.f. [3], [2]):

Theorem 4. [8] Given a 1-reduced simplicial set $X$, there is a canonical identification isomorphism

$$
\Omega C_{*}(X) \approx C_{*}^{\square}(\Omega X) .
$$

Theorem 5. [9] Given a 1-reduced cubical set $Q$, there is a canonical identification isomorphism

$$
\Omega C_{*}^{\square}(Q) \approx C_{*}^{\diamond}(\boldsymbol{\Omega} Q) .
$$


For completeness, definitions of these two functors appear in the appendix. Since the chain complex of any cubical set $Q$ is a DG coalgebra with strictly coassociative coproduct, setting $Q=\boldsymbol{\Omega} X$ in Theorem 5 immediately gives:

Theorem 6. For a 2-reduced simplicial set $X$ there is a canonical identification isomorphism

$$
\Omega^{2} C_{*}(X) \approx C_{*}^{\diamond}\left(\boldsymbol{\Omega}^{2} X\right) .
$$

Now if $X=\operatorname{Sing}^{1} Y$, then $\Omega C_{*}(X)$ is Adams' cobar construction for the space $Y[\mathbf{1}]$; consequently, there is a canonical (geometric) coproduct on $\Omega^{2} C_{*}\left(\operatorname{Sing}^{1} Y\right)$. We shall extend this canonical coproduct to an " $A_{\infty}$-Hopf algebra" structure in the sequel $[\mathbf{1 6}]$.

\section{The Multiplihedra and Associahedra}

The multiplihedron $J_{n}$ and the associahedron $K_{n+1}$ are cellular projections of $P_{n}$ defined in terms of planar trees. Consequently, we shall need to index the faces of $P_{n}$ four ways: (1) by partitions of $\underline{n}$ (see Section 2), (2) by (planar rooted) $p$-leveled trees with $n+1$ leaves (PLT's), (3) by parenthesized strings of $n+1$ indeterminants with $p-1$ levels of subscripted parentheses and (4) by $(p-1)$-fold compositions of face operators acting on $n+1$ indeterminants. The second and third serve as transitional intermediaries between the first and fourth.

Define a correspondence between PLT's and partitions of $\underline{n}$ as follows: Let $T_{n+1}^{p}$ be a PLT with $n+1$ leaves, $p$-levels and root in level $p$. Number the leaves from left to right and assign the label $i$ to the node at which the branch of leaf $i$ meets the branch of leaf $i+1,1 \leqslant i \leqslant n$ (a node may have multiple labels). Let $U_{j}=$ \{labels assigned to $j$-level nodes\} and identify $T_{n+1}^{p}$ with the partition $U_{1}|\cdots| U_{p}$ of $\underline{n}$ (see Figure 9 ). Thus binary $n$-leveled trees parametrize the vertices of $P_{n}$. Loday and Ronco constructed a map from $S_{n}$ to binary $n$-leveled trees [12]; its extension to faces of $P_{n}$ was given by Tonks [19]. Note that the map from PLT's to partitions defined above gives an inverse.

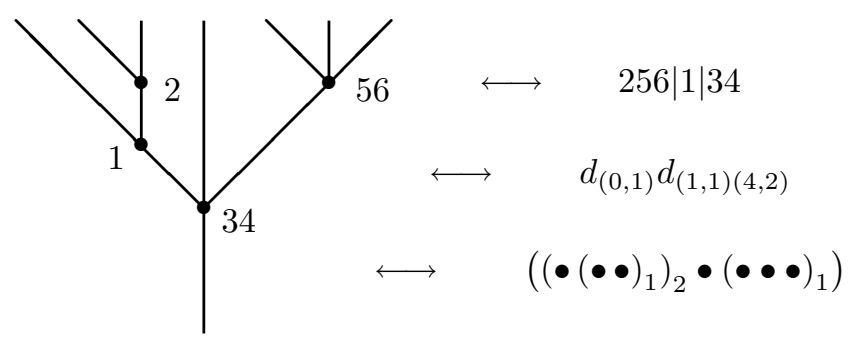

Figure 9: Various representations of the face 256|1|34.

To define the correspondence between PLT's and subscripted parenthesizations of $n+1$ indeterminants, begin by identifying the top cell of $P_{n}$ with the $(n+1)$-leaf corolla and the (unsubscripted) parenthesized string $\left(x_{1} x_{2} \cdots x_{n+1}\right)$. Let $T_{n+1}^{p}$ be a 
PLT with $p>1$. If the branches meeting at a level 1 node contain leaves $i, \ldots, i+k$, enclose the corresponding indeterminants in a pair of parentheses with subscript 1 ; if the branches meeting at a level 2 node contain leaves $i_{1}, \ldots, i_{k}$, enclose the corresponding indeterminants in a pair of parentheses with subscript 2 ; and so on for $p-1$ steps (see Example 5).

Compositions of face operators encode this parenthesization procedure. For $s \geqslant 1$, choose $s$ pairs of indices $\left(i_{1}, \ell_{1}\right) \cdots\left(i_{s}, \ell_{s}\right)$ such that $0 \leqslant i_{r}<i_{r+1} \leqslant n-1$ and $i_{r}+\ell_{r}+1 \leqslant i_{r+1}$. The face operator

$$
d_{\left(i_{1}, \ell_{1}\right) \cdots\left(i_{s}, \ell_{s}\right)}: P_{n} \rightarrow \partial P_{n}
$$

acts on $\left(x_{1} x_{2} \cdots x_{n+1}\right)$ by simultaneously inserting $s$ disjoint (non-nested) pairs of inner parentheses with subscript 1 , the first enclosing $x_{i_{1}+1} \cdots x_{i_{1}+\ell_{1}+1}$, the second enclosing $x_{i_{2}+1} \cdots x_{i_{2}+\ell_{2}+1}$, and so on. Thus,

$$
\begin{aligned}
& d_{\left(i_{1}, \ell_{1}\right) \cdots\left(i_{s}, \ell_{s}\right)}\left(x_{1} x_{2} \cdots x_{n+1}\right)= \\
&\left(x_{1} \cdots\left(x_{i_{1}+1} \cdots x_{i_{1}+\ell_{1}+1}\right)_{1} \cdots\left(x_{i_{s}+1} \cdots x_{i_{s}+\ell_{s}+1}\right)_{1} \cdots x_{n+1}\right) .
\end{aligned}
$$

A composition of face operators

$$
d_{\left(i_{1}^{k}, \ell_{1}^{k}\right) \cdots\left(i_{s_{k}}^{k}, \ell_{s_{k}}^{k}\right)} \cdots d_{\left(i_{1}^{1}, \ell_{1}^{1}\right) \cdots\left(i_{s_{1}}^{1}, \ell_{s_{1}}^{1}\right)}: P_{n} \rightarrow \partial^{k} P_{n}
$$

continues this process inductively: If the $j^{\text {th }}$ operator inserted parentheses with subscript $j$, treat each such pair and its contents as a single indeterminant and apply the $(j+1)^{s t}$ as above, inserting parentheses subscripted by $j+1$.

Refer to Figure 9 above. The composition $d_{(0,1)} d_{(1,1)(4,2)}$ acts on $(\bullet \bullet \bullet \bullet \bullet \bullet)$ in the following way: First, $d_{(1,1)(4,2)}$ simultaneously inserts two inner pairs of parentheses with subscript 1 :

$$
\left(\bullet(\bullet \bullet)_{1} \bullet(\bullet \bullet \bullet)_{1}\right) \text {. }
$$

Next, $d_{(0,1)}$ inserts the single pair with subscript 2:

$$
\left(\left(\bullet(\bullet \bullet)_{1}\right)_{2} \bullet(\bullet \bullet \bullet)_{1}\right)
$$

We summarize the discussion above as a proposition.

Proposition 5. The following correspondences (defined above) preserve combinatorial structure:

$$
\begin{aligned}
\left\{\text { Faces of } P_{n}\right\} & \leftrightarrow \quad\{\text { Partitions of } \underline{n}\} \\
& \leftrightarrow\{\text { Leveled trees with } n+1 \text { leaves }\} \\
& \leftrightarrow\left\{\begin{array}{c}
\text { Strings of } n+1 \text { indeterminants } \\
\text { with subscripted parentheses }
\end{array}\right\} \\
& \leftrightarrow\left\{\begin{array}{c}
\text { Compositions of face operators } \\
\text { acting on } n+1 \text { indeterminants. }
\end{array}\right\}
\end{aligned}
$$

Assign the identity face operator $I d$ to the top dimensional face of $P_{n+1}$ and use the correspondences above to assign compositions of faces operators to lower 
dimensional faces (see Figure 10). For faces in codimension 1 we have:

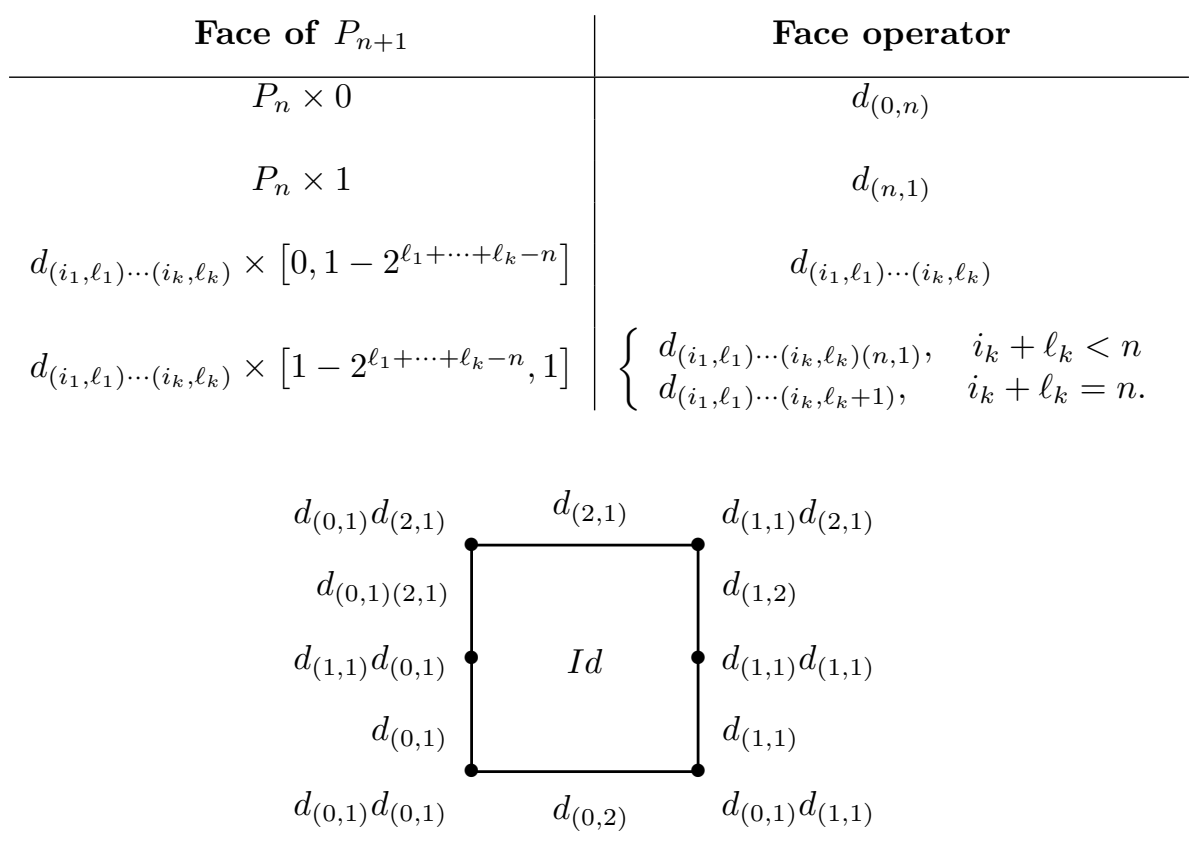

Figure 10: $P_{3}$ with face-operator labeling.

Since compositions of face operators are determined by the correspondence between faces and partitions, we only label the codimension 1 faces of the related polytopes below.

The associahedra $\left\{K_{n}\right\}$ serve as parameter spaces for higher homotopy associativity. In his seminal papers of 1963 [18], J. Stasheff constructed $K_{n}$ in the following way: Let $K_{2}=*$; if $K_{n-1}$ has been constructed, define $K_{n}$ to be the cone on the set

$$
\bigcup_{\substack{r+s=n+1 \\ 1 \leqslant k \leqslant n-s+1}}\left(K_{r} \times K_{s}\right)_{k} .
$$

Thus, $K_{n}$ is an $(n-2)$-dimensional convex polytope.

Stasheff's motivating example of higher homotopy associativity in $[\mathbf{1 8}]$ is the singular chain complex on the (Poincarè) loop space of a connected $C W$-complex. Here associativity holds up to homotopy, the homotopies between the various associations are homotopic, the homotopies between these homotopies are homotopic, and so on. An abstract $A_{\infty}$-algebra is a DGA in which associativity behaves as in Stasheff's motivating example. If $\varphi^{2}: A \otimes A \rightarrow A$ is the multiplication on an $A_{\infty}$-algebra $A$, the homotopies $\varphi^{n}: A^{\otimes n} \rightarrow A$ are multilinear operations such that $\varphi^{3}$ is a chain homotopy between the associations $(a b) c$ and $a(b c)$ thought of as quadratic compositions $\varphi^{2}\left(\varphi^{2} \otimes 1\right)$ and $\varphi^{2}\left(1 \otimes \varphi^{2}\right)$ in three variables, $\varphi^{4}$ is a chain homotopy bounding the cycle of five quadratic compositions in four variables involving $\varphi^{2}$ and 
$\varphi^{3}$, and so on. Let $C_{*}\left(K_{r}\right)$ denote the cellular chains on $K_{r}$. The natural correspondence between faces of $K_{r}$ and the various compositions of the $\varphi^{n}$ 's in $r$ variables (modulo an appropriate equivalence) induces a chain map $C_{*}\left(K_{r}\right) \rightarrow \operatorname{Hom}\left(A^{\otimes r}, A\right)$ that determines the relations among the compositions of $\varphi^{n}$ 's. This chain map together with our diagonal on $K_{n}$ leads to the tensor product of $A_{\infty}$-algebras (see Section 5).

Now if we disregard levels, a PLT is simply a planar rooted tree (PRT). Quite remarkably, A. Tonks $[\mathbf{1 9}]$ showed that $K_{n}$ is the identification space $P_{n-1} / \sim$ in which all faces indexed by isomorphic PRT's are identified. Since the quotient map $\theta: P_{n-1} \rightarrow K_{n}$ is cellular, the faces of $K_{n}$ are indexed by PRT's with $n$ leaves. The correspondence between PRT's with $n$ leaves and parenthesizations of $n$ indeterminants is simply this: Given a node $N$, parenthesize the indeterminants that correspond to leaves on all branches that meet at node $N$.

Example 7. With one exception, all classes of faces of $P_{3}$ consist of a single element. Elements of the exceptional class

$$
[1|3| 2,13|2,3| 1 \mid 2]
$$

represent the parenthesization $((\bullet \bullet)(\bullet \bullet))$. Whereas $1|3| 2$ and $3|1| 2$ insert inner parentheses in the opposite order, the element $13 \mid 2$ inserts inner parentheses simultaneously and represents a homotopy between $1|3| 2$ and $3|1| 2$. Tonks' projection $\theta$ sends the exceptional class to the vertex of $K_{4}$ represented by the parenthesization $((\bullet \bullet)(\bullet \bullet))$. The classes of faces of $P_{4}$ with more than one element and their projections to $K_{5}$ are:

$$
\begin{aligned}
& {[12|4| 3,124|3,4| 12 \mid 3] } \stackrel{\theta}{\longrightarrow}((\bullet \bullet \bullet)(\bullet \bullet)) \\
& {[1|3| 24,13|24,3| 1 \mid 24] } \longrightarrow((\bullet)(\bullet \bullet) \\
& {[1|4| 23,14|23,4| 1 \mid 23] }\longrightarrow(\bullet \bullet) \bullet(\bullet \bullet)) \\
& {[2|4| 13,24|13,4| 2 \mid 13] } \longrightarrow(\bullet(\bullet \bullet)(\bullet \bullet)) \\
& {[1|34| 2,134|2,34| 1 \mid 2] } \longrightarrow((\bullet)(\bullet \bullet \bullet)) \\
& {[1|3| 2|4,13| 2|4,3| 1|2| 4] \longrightarrow(((\bullet \bullet)(\bullet \bullet)) \bullet) } \\
& {[2|4| 3|1,24| 3|1,4| 2|3| 1] \longrightarrow(\bullet((\bullet \bullet)(\bullet \bullet))) } \\
& {[1|2| 4|3,1| 24|3,1| 4|2| 3,14|2| 3,4|1| 2 \mid 3] \longrightarrow(((\bullet \bullet) \bullet)(\bullet \bullet)) } \\
& {[1|3| 4|2,13| 4|2,3| 1|4| 2,3|14| 2,3|4| 1 \mid 2] \longrightarrow((\bullet \bullet)((\bullet \bullet))} \\
& {[1|4| 3|2,14| 3|2,4| 1|3| 2,4|13| 2,4|3| 1 \mid 2] \longrightarrow((\bullet \bullet)(\bullet(\bullet \bullet))) } \\
& {[2|1| 4|3,2| 14|3,2| 4|1| 3,24|1| 3,4|2| 1 \mid 3] \longrightarrow((\bullet(\bullet \bullet))(\bullet \bullet)) . }
\end{aligned}
$$

Faces and edges represented by elements of the first five classes project to edges; edges and vertices represented by elements of the next six classes project to vertices.

The multiplihedra $\left\{J_{n+1}\right\}$, which serve as parameter spaces for homotopy multiplicative morphisms of $A_{\infty}$-algebras, lie between the associahedra and permutahedra (see [18], [6]). If $f^{1}: A \rightarrow B$ is such a morphism, there is a chain homotopy $f^{2}$ between the quadratic compositions $f^{1} \varphi_{A}^{2}$ and $\varphi_{B}^{2}\left(f^{1} \otimes f^{1}\right)$ in two variables, there is a chain homotopy $f^{3}$ bounding the cycle of the six quadratic compositions in three variables involving $f^{1}, f^{2}, \varphi_{A}^{2}, \varphi_{A}^{3}, \varphi_{B}^{2}$ and $\varphi_{B}^{3}$, and so on. The 
natural correspondence between faces of $J_{r}$ and the various compositions of $f^{i}, \varphi_{A}^{j}$ and $\varphi_{B}^{k}$ in $r$ variables (modulo an appropriate equivalence) induces a chain map $C_{*}\left(J_{r}\right) \rightarrow \operatorname{Hom}\left(A^{\otimes r}, B\right)$.

The multiplihedron $J_{n+1}$ can also be realized as a subdivision of the cube $I^{n}$. For $n=0,1,2$, set $J_{n+1}=P_{n+1}$. If $J_{n}$ has been constructed, $J_{n+1}$ is the subdivision of $J_{n} \times I$ given below and its various $(n-1)$-faces are labeled as indicated:

\begin{tabular}{c|c} 
Face of $J_{n+1}$ & Face operator \\
\hline$J_{n} \times 0$ & $d_{(0, n)}$ \\
$J_{n} \times 1$ & $d_{(n, 1)}$ \\
$d_{(i, \ell)} \times I$ & $1 \leqslant i<n-\ell$ \\
$d_{(i, \ell)} \times\left[0,1-2^{-i}\right]$ & $d_{(i, \ell)}, \quad 1 \leqslant i=n-\ell$ \\
$d_{(i, \ell)} \times\left[1-2^{-i}, 1\right]$ & $d_{(i, \ell)}, \quad 1 \leqslant i=n-\ell$ \\
$d_{\left(0, \ell_{1}\right) \cdots\left(i_{k}, \ell_{k}\right)} \times\left[0,1-2^{k-n}\right]$ & $d_{(i, \ell+1)}, 1 \leqslant i=n$ \\
$d_{\left(0, \ell_{1}\right) \cdots\left(i_{k}, \ell_{k}\right)} \times\left[1-2^{k-n}, 1\right]$ & $d_{\left(0, \ell_{1}\right) \cdots\left(i_{k}, \ell_{k}\right)}$
\end{tabular}

Thus faces of $J_{n+1}$ are indexed by compositions of face operators of the form

$$
d_{\left(i_{m}, \ell_{m}\right)} \cdots d_{\left(i_{k_{1}}, \ell_{k_{1}}\right) \cdots\left(i_{k_{s}}, \ell_{k_{s}}\right)} \cdots d_{\left(i_{1}, \ell_{1}\right)} .
$$

In terms of trees and parenthesizations this says the following: Let $T$ be a $(k+1)$ leveled tree with left-most branch attached at level $p$. For $1 \leqslant j<p$, insert level $j$ parentheses one pair at a time without regard to order as in $K_{n+2}$; next, insert all level $p$ parentheses simultaneously as in $P_{n+1}$; finally, for $j>p$, insert level $j$ parentheses one pair at a time without regard to order. Thus multiple lower indices in a composition of face operators may only occur when the left-most branch is attached above the root. This suggests the following equivalence relation on the set of $(k+1)$-leveled trees with $n+2$ leaves: Let $T$ and $T^{\prime}$ be $p$-leveled trees with $n+2$ nodes whose $p$-level meets $U_{p}$ and $U_{p}^{\prime}$ contain 1 . Then $T \sim T^{\prime}$ if $T$ and $T^{\prime}$ are isomorphic as PLT's and $U_{p}=U_{p}^{\prime}$. This equivalence relation induces a cellular projection $\pi: P_{n+1} \rightarrow J_{n+1}$ under which $J_{n+1}$ can be realized as an identification space of $P_{n+1}$. Furthermore, the projection $J_{n+1} \rightarrow K_{n+2}$ given by identifying faces of $J_{n+1}$ indexed by isomorphic PLT's gives the factorization $P_{n+1} \stackrel{\pi}{\longrightarrow} J_{n+1} \rightarrow K_{n+2}$ of Tonks' projection.

It is interesting to note the role of the indices $\ell_{j}$ in compositions of face operators representing the faces of $J_{n+1}$ as in (5.2). With one exception, each $U_{j}$ in the corresponding partition $U_{1}|\cdots| U_{m+1}$ is a set of consecutive integers; this holds without exception for all $U_{j}$ on $K_{n+2}$. The exceptional set $U_{p}$ is a union of $s$ sets of consecutive integers with maximal cardinality, as is typical of sets $U_{j}$ on $P_{n+1}$. Thus $J_{n+1}$ exhibits characteristics of both combinatorial structures. 


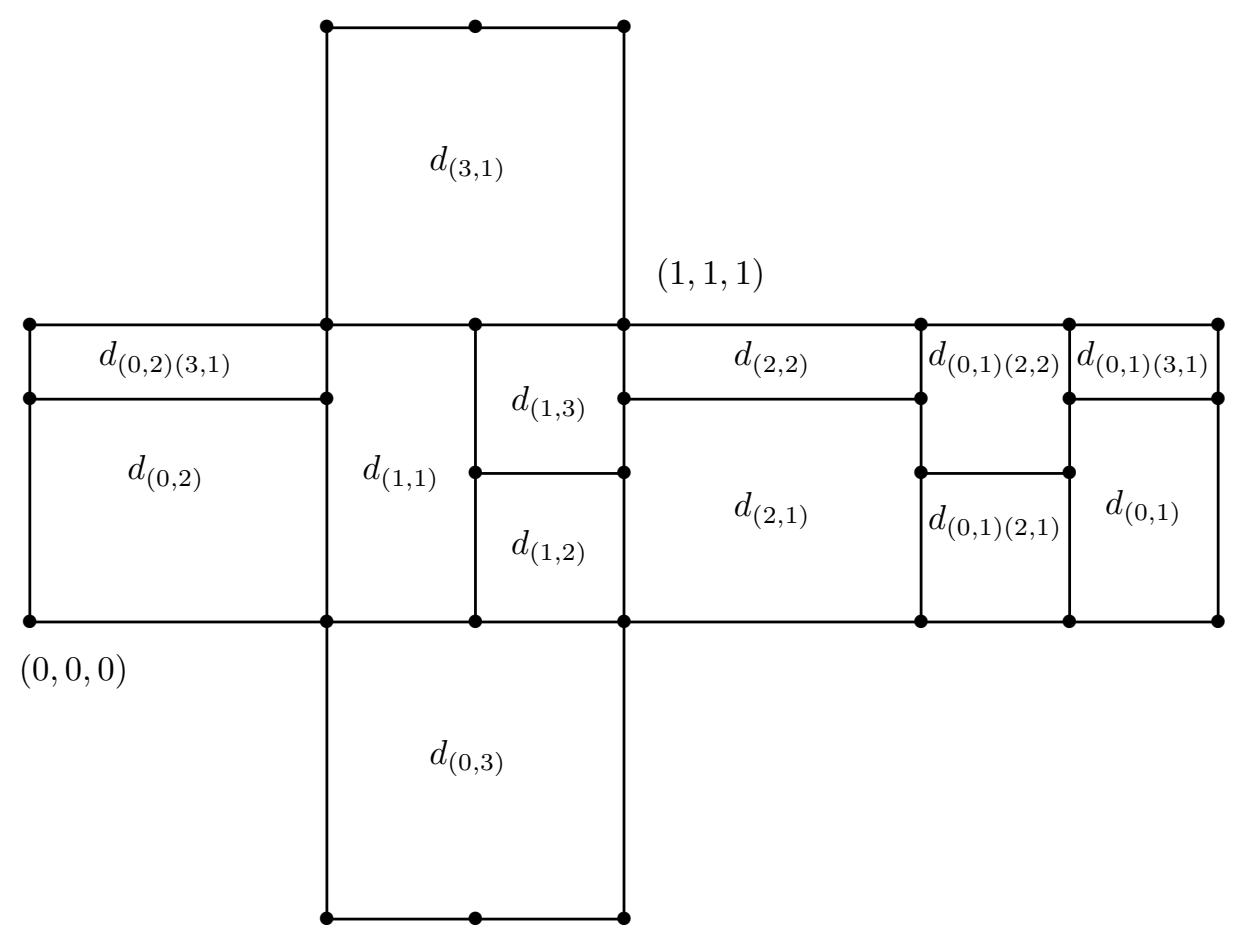

Figure 11: $J_{4}$ as a subdivision of $J_{3} \times I$.

We realize the associahedron $K_{n+2}$ in a similar way. For $n=0,1$, set $K_{n+2}=$ $P_{n+1}$. If $K_{n+1}$ has been constructed, let $e_{i, \epsilon}$ denote the face $\left(x_{1}, \ldots, x_{i-1}, \epsilon, x_{i+1}\right.$, $\left.\ldots, x_{n}\right) \subset I^{n}$, where $\epsilon=0,1$ and $1 \leqslant i \leqslant n$. Then $K_{n+2}$ is the subdivision of $K_{n+1} \times I$ given below and its various $(n-1)$-faces are labeled as indicated:

\begin{tabular}{l|l} 
Face of $K_{n+2}$ & Face operator
\end{tabular}

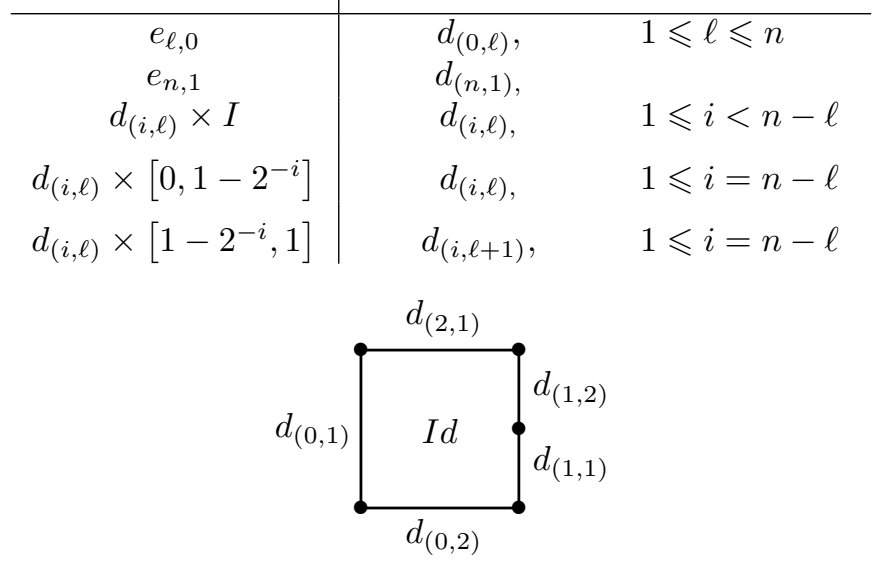

Figure 12: $K_{4}$ as a subdivision of $K_{3} \times I$. 


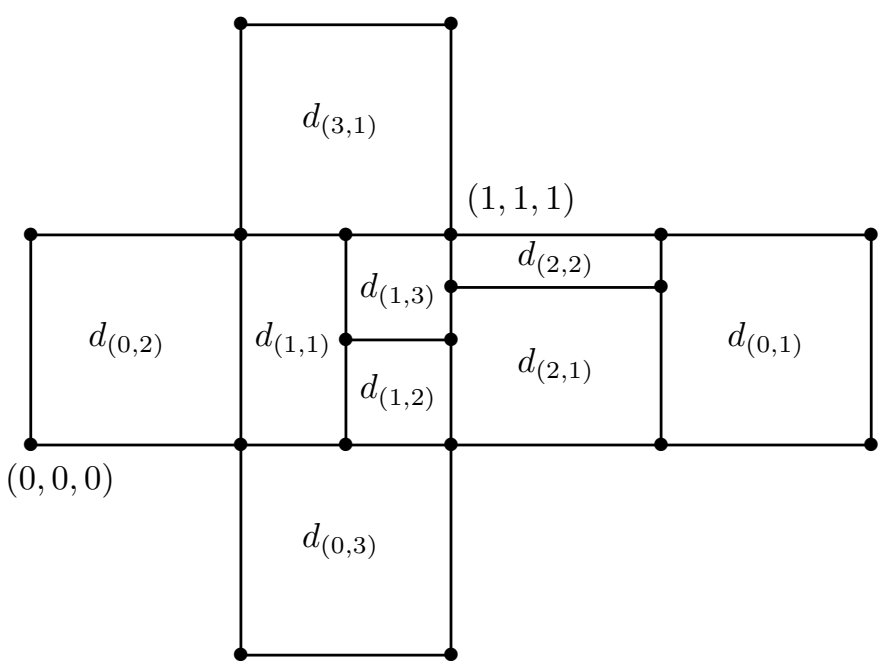

Figure 13: $K_{5}$ as a subdivision of $K_{4} \times I$.

\section{Diagonals on the Associahedra and Multiplihedra}

The diagonal $\Delta_{P}$ on $C_{*}\left(P_{n+1}\right)$ descends to diagonals $\Delta_{J}$ on $C_{*}\left(J_{n+1}\right)$ and $\Delta_{K}$ on $C_{*}\left(K_{n+2}\right)$ via the cellular projections $\pi: P_{n+1} \rightarrow J_{n+1}$ and $\theta: P_{n+1} \rightarrow K_{n+2}$ discussed in Section 2 above. This fact is an immediate consequence of Proposition 7 .

Definition 20. Let $f: W \rightarrow X$ be a cellular map of $C W$-complexes, let $\Delta_{W}$ be a diagonal on $C_{*}(W)$ and let $X^{(r)}$ denote the r-skeleton of $X$. A $k$-cell $e \subseteq W$ is degenerate under $f$ if $f(e) \subseteq X^{(r)}$ with $r<k$. A component $a \otimes b$ of $\Delta_{W}$ is degenerate under $f$ if either $a$ or $b$ is degenerate under $f$.

Let us identify the non-degenerate cells of $P_{n+1}$ under $\pi$ and $\theta$.

Definition 21. Let $A_{1}|\cdots| A_{p}$ be a partition of $\underline{n+1}$ with $p>1$ and let $1 \leqslant k<$ $p$. The subset $A_{k}$ is exceptional if for $k<j \leqslant p$, there is an element $a_{i, j} \in A_{j}$ such that $\min A_{k}<a_{i, j}<\max A_{k}$.

Proposition 6. Let $a=A_{1}|\cdots| A_{p}$ be a face of $P_{n+1}$ and let

$$
d_{\left(i_{1}^{p-1}, \ell_{1}^{p-1}\right) \cdots\left(i_{s_{p-1}}^{p-1}, \ell_{s_{p-1}}^{p-1}\right)} \cdots d_{\left(i_{1}^{1}, \ell_{1}^{1}\right) \cdots\left(i_{s_{1}}^{1}, \ell_{s_{1}}^{1}\right)}
$$

be its unique representation as a composition of face operators.

(1) The following are all equivalent:

(1a) The face a is degenerate under $\pi$.

(1b) $\min A_{j}>\min \left(A_{j+1} \cup \cdots \cup A_{p}\right)$ with $A_{j}$ exceptional for some $j<p$.

(1c) $i_{1}^{k}>0$ and $s_{k}>1$ for some $k<p$. 
(2) The following are all equivalent:

(2a) The face a is degenerate under $\theta$.

(2b) $A_{j}$ is exceptional for some $j<p$.

(2c) $s_{k}>1$ for some $k<p$.

Proof. Obvious.

Example 8. The subset $A_{1}=\{13\}$ in the partition $a=13 \mid 24$ is exceptional and the face $a \subset P_{4}$ is degenerate under $\theta$. In terms of compositions of face operators, the face a corresponds to $d_{(0,1)(2,1)}\left(x_{1} \cdots x_{5}\right)$ with $s_{1}=2$. Furthermore, a is also non-degenerate under $\pi$ since $i_{1}^{1}=0$ (equivalently, $\min A_{1}<\min A_{2}$ ).

Next, we apply Tonks' projection and obtain an explicit formula for the diagonal $\Delta_{K}$ on the associahedra.

Proposition 7. Let $f: W \rightarrow X$ be a surjective cellular map and let $\Delta_{W}$ be a diagonal on $C_{*}(W)$. Then $\Delta_{W}$ uniquely determines a diagonal $\Delta_{X}$ on $C_{*}(X)$ given by the non-degenerate components of $\Delta_{W}$ under $f$. Moreover, $\Delta_{X}$ is the unique map that commutes the following diagram:

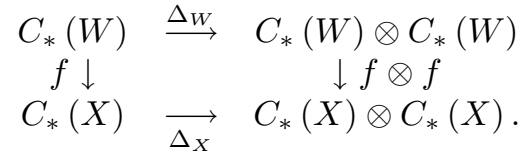

Proof. Obvious.

In Section 2 we established correspondences between faces of $P_{n+1}$ and PLT's with $n+2$ leaves and between faces of $K_{n+2}$ and PRT's with $n+2$ leaves. Since a PRT can be viewed as a PLT, faces of $K_{n+2}$ can be viewed as faces of $P_{n+1}$.

Definition 22. For $n \geqslant 0$, let $\Delta_{P}$ be the diagonal on $C_{*}\left(P_{n+1}\right)$ and let $\theta: P_{n+1} \rightarrow$ $K_{n+2}$ be Tonks' projection. View each face $e$ of the associahedron $K_{n+2}$ as a face of $P_{n+1}$ and define $\Delta_{K}: C_{*}\left(K_{n+2}\right) \rightarrow C_{*}\left(K_{n+2}\right) \otimes C_{*}\left(K_{n+2}\right)$ by

$$
\Delta_{K}(e)=(\theta \otimes \theta) \Delta_{P}(e)
$$

Corollary 2. The map $\Delta_{K}$ given by Definition 22 is the diagonal on $C_{*}\left(K_{n+2}\right)$ induced by $\Delta_{P}$.

Proof. This is an immediate application of Proposition 7.

Consider a CP $u \otimes v=c(F) \otimes r(F)$ related to $\mathrm{SCP} a \otimes b=c(E) \otimes r(E)$ via $F=D_{N_{q-1}} \cdots D_{1} R_{M_{p-1}} \cdots R_{1} E$. Note that both factors of $u \otimes v$ are nondegenerate under $\theta$ if and only if $b$ is non-degenerate and each $M_{j}$ has maximal cardinality. Alternatively, if $d^{p} \cdots d^{1} \otimes d^{q} \cdots d^{1}\left(e^{n} \otimes e^{n}\right)$ is a component of $\Delta_{K}\left(e^{n}\right)$, factors in the corresponding pairing $T_{p} \otimes T_{q}$ of PRT's have $n+2$ leaves, $p+1$ and $q+1$ nodes and respective dimensions $n-p$ and $n-q$. Hence $p+q=n$ and $T_{p} \otimes T_{q}$ 
has exactly $n+2$ nodes. But if $T_{u} \otimes T_{v}$ is the pairing of PLT's corresponding to $u \otimes v$, forgetting levels in $T_{u} \otimes T_{v}$ gives the pairing of PRT's corresponding to $\theta(u) \otimes \theta(v)$. Since the number of nodes in $T_{u} \otimes T_{v}$ is at least $n+2, \theta(a) \otimes \theta(b)$ is non-degenerate in $\Delta_{K}\left(e^{n}\right)$ if and only if the total number of nodes in $T_{u} \otimes T_{v}$ is exactly $n+2$.

Choose a system of generators $e^{n} \in C_{n}\left(K_{n+2}\right), n \geqslant 0$. The signs in (6.1) below follow from (3.2).

Definition $23([\mathbf{1 5}])$. For each $n \geqslant 0$, define $\Delta_{K}$ on $e^{n} \in C_{n}\left(K_{n+2}\right)$ by

$\Delta_{K}\left(e^{n}\right)=\sum_{0 \leqslant p \leqslant p+q=n+2}(-1)^{\epsilon} d_{\left(i_{p-1}, \ell_{p-1}\right)} \cdots d_{\left(i_{1}, \ell_{1}\right)} \otimes d_{\left(i_{q-1}^{\prime}, \ell_{q-1}^{\prime}\right)} \cdots d_{\left(i_{1}^{\prime}, \ell_{1}^{\prime}\right)}\left(e^{n} \otimes e^{n}\right)$,

where

$$
\epsilon=\sum_{j=1}^{p-1} i_{j}\left(\ell_{j}+1\right)+\sum_{k=1}^{q-1}\left(i_{k}^{\prime}+k+q\right) \ell_{k}^{\prime},
$$

and lower indices $\left(\left(i_{1}, \ell_{1}\right), \ldots,\left(i_{p-1}, \ell_{p-1}\right) ;\left(i_{1}^{\prime}, \ell_{1}^{\prime}\right), \ldots,\left(i_{q-1}^{\prime}, \ell_{q-1}^{\prime}\right)\right)$ range over all solutions of the following system of inequalities:

$$
\left\{\begin{array}{ll}
1 \leqslant i_{j}^{\prime}<i_{j-1}^{\prime} \leqslant n+1 & \text { (1) } \\
1 \leqslant \ell_{j}^{\prime} \leqslant n+1-i_{j}^{\prime}-\ell_{(j-1)}^{\prime} & (2) \\
0 \leqslant i_{k} \leqslant \min _{o^{\prime}\left(t_{k}\right)<r<k}\left\{i_{r}, i_{t_{k}}^{\prime}-\ell_{\left(o^{\prime}\left(t_{k}\right)\right)}\right\} & \text { (3) } \\
1 \leqslant \ell_{k}=\epsilon_{k}-i_{k}-\ell_{(k-1)}, & \text { (4) }
\end{array}\right\}_{\substack{1 \leqslant k \leqslant p-1 \\
1 \leqslant j \leqslant q-1}}
$$

where

$$
\begin{aligned}
& \left\{\epsilon_{1}<\cdots<\epsilon_{q-1}\right\}=\{1, \ldots, n\} \backslash\left\{i_{1}^{\prime}, \ldots, i_{q-1}^{\prime}\right\} ; \\
& \epsilon_{0}=\ell_{0}=\ell_{0}^{\prime}=i_{p}=i_{q}^{\prime}=0 ; \\
& i_{0}=i_{0}^{\prime}=\epsilon_{q}=\ell_{(p)}=\ell_{(q)}^{\prime}=n+1 ; \\
& \ell_{(u)}=\sum_{j=0}^{u} \ell_{j} \text { for } 0 \leqslant u \leqslant p ; \\
& \ell_{(u)}^{\prime}=\sum_{k=0}^{u} \ell_{k}^{\prime} \text { for } 0 \leqslant u \leqslant q ; \\
& t_{u}=\min \left\{r \mid i_{r}^{\prime}+\ell_{(r)}^{\prime}-\ell_{(o(u))}^{\prime}>\epsilon_{u}>i_{r}^{\prime}\right\} ; \\
& o(u)=\max \left\{r \mid i_{r}^{\prime} \geqslant \epsilon_{u}\right\} ; \text { and } \\
& o^{\prime}(u)=\max \left\{r \mid \epsilon_{r} \leqslant i_{u}^{\prime}\right\} .
\end{aligned}
$$

Extend $\Delta_{K}$ to proper faces of $K_{n+2}$ via the standard comultiplicative extension.

Theorem 7. The map $\Delta_{K}$ given by Definition 23 is the diagonal induced by $\theta$. 
Proof. If $v=L_{\beta}\left(v^{\prime}\right)$ is non-degenerate in some component $u \otimes v$ of $\Delta_{P}$, then so is $v^{\prime}$, and we immediately obtain inequality (1) of (6.2). Next, each non-degenerate decreasing $b$ uniquely determines an SCP $a \otimes b$. Although $a$ may be degenerate, there is a unique non-degenerate $u=R_{M_{p-1}} \cdots R_{M_{1}}(a)$ obtained by choosing each $M_{j}$ with maximal cardinality (the case $M_{j}=\varnothing$ for all $j$ may nevertheless occur); then $u \otimes b$ is a non-degenerate CP associated with $a \otimes b$ in $\Delta_{P}$. As a composition of face operators, straightforward examination shows that $u$ has form $u=d_{\left(i_{p-1}, \ell_{p-1}\right)} \cdots d_{\left(i_{1}, \ell_{1}\right)}\left(e^{n}\right)$ and is related to $b=d_{\left(i_{q-1}^{\prime}, \ell_{q-1}^{\prime}\right)} \cdots d_{\left(i_{1}^{\prime}, \ell_{1}^{\prime}\right)}\left(e^{n}\right)$ by

$$
i_{k}=\min _{o^{\prime}\left(t_{k}\right)<r<k}\left\{i_{r}, i_{t_{k}}^{\prime}-\ell_{\left(o^{\prime}\left(t_{k}\right)\right)}\right\}, 1 \leqslant k<p ;
$$

and equality holds in (4) of (6.2). Finally, let $b=L_{\beta}(\bar{b})$. As we vary $\bar{b}$ in all possible ways, each $\bar{b}$ is non-degenerate and we obtain all possible non-degenerate CP's $\bar{u} \otimes \bar{b}$ associated with $\bar{a} \otimes \bar{b}(\bar{u}=u$ when $\bar{b}=b$ and $\beta=\varnothing)$. For each such $\bar{u}=d_{\left(i_{p-1}, \ell_{p-1}\right)} \cdots d_{\left(i_{1}, \ell_{1}\right)}\left(e^{n}\right)$ we have both inequality (3) and equality in (4) of (6.2). Hence, the theorem is proved.

Example 9. On $K_{4}$ we obtain:

$$
\begin{aligned}
\Delta_{K}\left(e^{2}\right)=\left\{d_{(0,1)}\right. & d_{(0,1)} \otimes 1+1 \otimes d_{(1,1)} d_{(2,1)}+d_{(0,2)} \otimes d_{(1,1)} \\
& \left.+d_{(0,2)} \otimes d_{(1,2)}+d_{(1,1)} \otimes d_{(1,2)}-d_{(0,1)} \otimes d_{(2,1)}\right\}\left(e^{2} \otimes e^{2}\right) .
\end{aligned}
$$

\section{Application: Tensor Products of $A_{\infty}-($ co)algebras}

In this section, we use $\Delta_{K}$ to define the tensor product of $A_{\infty}$-(co)algebras in maximal generality. We note that a special case was given by J. Smith [17] for certain objects with a richer structure than we have here. We also mention that Lada and Markl [11] defined an $A_{\infty}$ tensor product structure on a construct different from the tensor product of graded modules.

We adopt the following notation and conventions: Let $R$ be a commutative ring with unity; $R$-modules are assumed to be $\mathbb{Z}$-graded, tensor products and Hom's are defined over $R$ and all maps are $R$-module maps unless otherwise indicated. If an $R$-module $V$ is connected, $\bar{V}=V / V_{0}$. The symbol $1: V \rightarrow V$ denotes the identity map; the suspension and desuspension maps $\uparrow$ and $\downarrow$ shift dimension by +1 and -1 , respectively. Define $V^{\otimes 0}=R$ and $V^{\otimes n}=V \otimes \cdots \otimes V$ with $n>0$ factors; then $T V=\oplus_{n \geqslant 0} V^{\otimes n}$ and $T^{a} V$ (respectively, $T^{c} V$ ) denotes the free tensor algebra (respectively, cofree tensor coalgebra) of $V$. Given $R$-modules $V_{1}, \ldots, V_{n}$, a permutation $\sigma \in S_{n}$ induces an isomorphism $\sigma: V_{1} \otimes \cdots \otimes V_{n} \rightarrow V_{\sigma^{-1}(1)} \otimes \cdots \otimes V_{\sigma^{-1}(n)}$ by $\sigma\left(x_{1} \cdots x_{n}\right)= \pm x_{\sigma^{-1}(1)} \cdots x_{\sigma^{-1}(n)}$, where \pm is the Koszul sign. In particular, $\sigma_{2, n}=$ $(13 \cdots(2 n-1) 24 \cdots 2 n):(A \otimes B)^{\otimes n} \rightarrow A^{\otimes n} \otimes B^{\otimes n}$ and $\sigma_{n, 2}=\sigma_{2, n}^{-1}$ induce isomorphisms $\left(\sigma_{2, n}\right)^{*}: \operatorname{Hom}\left(A^{\otimes n} \otimes B^{\otimes n}, A \otimes B\right) \rightarrow \operatorname{Hom}\left((A \otimes B)^{\otimes n}, A \otimes B\right)$ and $\left(\sigma_{n, 2}\right)_{*}: H o m\left(A \otimes B, A^{\otimes n} \otimes B^{\otimes n}\right) \rightarrow \operatorname{Hom}\left(A \otimes B, \quad(A \otimes B)^{\otimes n}\right)$. The map $\iota: \operatorname{Hom}(U, V) \otimes \operatorname{Hom}\left(U^{\prime}, V^{\prime}\right) \rightarrow \operatorname{Hom}\left(U \otimes U^{\prime}, V \otimes V^{\prime}\right)$ is the canonical isomorphism. If $f: V^{\otimes p} \rightarrow V^{\otimes q}$ is a map, we let $f_{i, n-p-i}=1^{\otimes i} \otimes f \otimes 1^{\otimes n-p-i}: V^{\otimes n} \rightarrow$ 
$V^{\otimes n-p+q}$, where $0 \leqslant i \leqslant n-p$. The abbreviations $D G M, D G A$, and $D G C$ stand for differential graded $R$-module, $D G R$-algebra and $D G R$-coalgebra, respectively.

We begin with a review of $A_{\infty}$-(co)algebras paying particular attention to the signs. Let $A$ be a connected $R$-module equipped with operations $\left\{\varphi^{k} \in\right.$ $\left.H_{o m}{ }^{k-2}\left(A^{\otimes k}, A\right)\right\}_{k \geqslant 1}$. For each $k$ and $n \geqslant 1$, linearly extend $\varphi^{k}$ to $A^{\otimes n}$ via

$$
\sum_{i=0}^{n-k} \varphi_{i, n-k-i}^{k}: A^{\otimes n} \rightarrow A^{\otimes n-k+1},
$$

and consider the induced map of degree -1 given by

$$
\sum_{i=0}^{n-k}\left(\uparrow \varphi^{k} \downarrow^{\otimes k}\right)_{i, n-k-i}:(\uparrow \bar{A})^{\otimes n} \rightarrow(\uparrow \bar{A})^{\otimes n-k+1} .
$$

Let $\widetilde{B} A=T^{c}(\uparrow \bar{A})$ and define a map $d_{\widetilde{B} A}: \widetilde{B} A \rightarrow \widetilde{B} A$ of degree -1 by

$$
d_{\widetilde{B} A}=\sum_{\substack{1 \leqslant k \leqslant n \\ 0 \leqslant i \leqslant n-k}}\left(\uparrow \varphi^{k} \downarrow^{\otimes k}\right)_{i, n-k-i} .
$$

The identities $(-1)^{[n / 2]} \uparrow^{\otimes n} \downarrow^{\otimes n}=1^{\otimes n}$ and $[n / 2]+[(n+k) / 2] \equiv n k+[k / 2](\bmod$ 2) imply that

$$
d_{\widetilde{B} A}=\sum_{\substack{1 \leqslant k \leqslant n \\ 0 \leqslant i \leqslant n-k}}(-1)^{[(n-k) / 2]+i(k+1)} \uparrow^{\otimes n-k+1} \varphi_{i, n-k-i}^{k} \downarrow^{\otimes n} .
$$

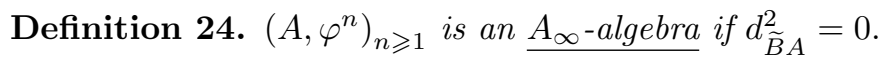

Proposition 8. For each $n \geqslant 1$, the operations $\left\{\varphi^{n}\right\}$ on an $A_{\infty}$-algebra satisfy the following quadratic relations:

$$
\sum_{\substack{0 \leqslant \ell \leqslant n-1 \\ 0 \leqslant i \leqslant n-\ell-1}}(-1)^{\ell(i+1)} \varphi^{n-\ell} \varphi_{i, n-\ell-1-i}^{\ell+1}=0 .
$$

Proof. For $n \geqslant 1$,

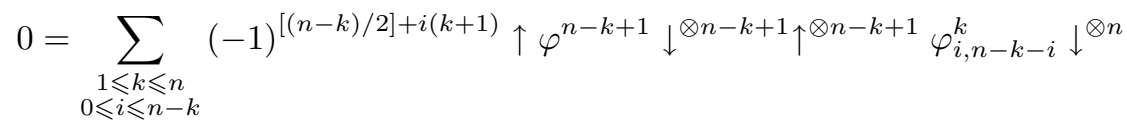

$$
\begin{aligned}
& =\sum_{\substack{1 \leqslant k \leqslant n \\
0 \leqslant i \leqslant n-k}}(-1)^{n-k+i(k+1)} \varphi^{n-k-1} \varphi_{i, n-k-i}^{k} \\
& =-(-1)^{n} \sum_{\substack{0 \leqslant \ell \leqslant n-1 \\
0 \leqslant i \leqslant n-\ell-1}}(-1)^{\ell(i+1)} \varphi^{n-\ell} \varphi_{i, n-\ell-1-i}^{\ell+1} .
\end{aligned}
$$

It is easy to prove that

Proposition 9. If $\left(A, \varphi^{n}\right)_{n \geqslant 1}$ is an $A_{\infty}$-algebra, then $\left(\widetilde{B} A, d_{\widetilde{B} A}\right)$ is a DGC. 
Definition 25. Let $\left(A, \varphi^{n}\right)_{n \geqslant 1}$ be an $A_{\infty}$-algebra. The tilde bar construction on $A$ is the $D G C\left(\widetilde{B} A, d_{\widetilde{B} A}\right)$.

Definition 26. Let $A$ and $C$ be $A_{\infty}$-algebras. A chain map $f=f^{1}: A \rightarrow C$ is a map of $A_{\infty}$-algebras if there is a sequence of maps $\left\{f^{k} \in \operatorname{Hom}^{k-1}\left(A^{\otimes k}, C\right)\right\}_{k \geqslant 2}$ such that

$$
\widetilde{f}=\sum_{n \geqslant 1}\left(\sum_{k \geqslant 1} \uparrow f^{k} \downarrow^{\otimes k}\right)^{\otimes n}: \widetilde{B} A \rightarrow \widetilde{B} C
$$

is a DGC map.

Dually, consider a sequence of operations $\left\{\psi^{k} \in \operatorname{Hom}^{k-2}\left(A, A^{\otimes k}\right)\right\}_{k \geqslant 1}$. For each $k$ and $n \geqslant 1$, linearly extend each $\psi^{k}$ to $A^{\otimes n}$ via

$$
\sum_{i=0}^{n-1} \psi_{i, n-1-i}^{k}: A^{\otimes n} \rightarrow A^{\otimes n+k-1},
$$

and consider the induced map of degree -1 given by

$$
\sum_{i=0}^{n-1}\left(\downarrow^{\otimes k} \psi^{k} \uparrow\right)_{i, n-1-i}:(\downarrow \bar{A})^{\otimes n} \rightarrow(\downarrow \bar{A})^{\otimes n+k-1} .
$$

Let $\widetilde{\Omega} A=T^{a}(\downarrow \bar{A})$ and define a map $d_{\widetilde{\Omega} A}: \widetilde{\Omega} A \rightarrow \widetilde{\Omega} A$ of degree -1 by

$$
d_{\widetilde{\Omega} A}=\sum_{\substack{n, k \geqslant 1 \\ 0 \leqslant i \leqslant n-1}}\left(\downarrow^{\otimes k} \psi^{k} \uparrow\right)_{i, n-1-i},
$$

which can be rewritten as

$$
d_{\widetilde{\Omega} A}=\sum_{\substack{n, k \geqslant 1 \\ 0 \leqslant i \leqslant n-1}}(-1)^{[n / 2]+i(k+1)+k(n+1)} \downarrow^{\otimes n+k-1} \psi_{i, n-1-i}^{k} \uparrow^{\otimes n} .
$$

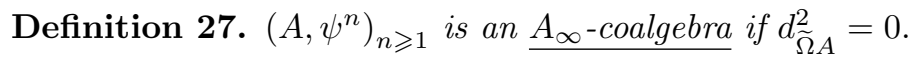

Proposition 10. For each $n \geqslant 1$, the operations $\left\{\psi^{k}\right\}$ on an $A_{\infty}$-coalgebra satisfy the following quadratic relations:

$$
\sum_{\substack{0 \leqslant \ell \leqslant n-1 \\ 0 \leqslant i \leqslant n-\ell-1}}(-1)^{\ell(n+i+1)} \psi_{i, n-\ell-1-i}^{\ell+1} \psi^{n-\ell}=0 .
$$

Proof. The proof is similar to the proof of Proposition 8 and is omitted.

Again, it is easy to prove that

Proposition 11. If $\left(A, \psi^{n}\right)_{n \geqslant 1}$ is an $A_{\infty}$-coalgebra, then $\left(\widetilde{\Omega} A, d_{\widetilde{\Omega} A}\right)$ is a DGA.

Definition 28. Let $\left(A, \psi^{n}\right)_{n \geqslant 1}$ be an $A_{\infty}$-coalgebra. The tilde cobar construction on Ais the $D G A\left(\widetilde{\Omega} A, d_{\widetilde{\Omega} A}\right)$. 
Definition 29. Let $A$ and $B$ be $A_{\infty}$-coalgebras. $A$ chain map $g=g^{1}: A \rightarrow B$ is a map of $A_{\infty}$-coalgebras if there is a sequence of maps $\left\{g^{k} \in H_{o m}^{k-1}\left(A, B^{\otimes k}\right)\right\}_{k \geqslant 2}$ such that

$$
\widetilde{g}=\sum_{n \geqslant 1}\left(\sum_{k \geqslant 1} \downarrow^{\otimes k} g^{k} \uparrow\right)^{\otimes n}: \widetilde{\Omega} A \rightarrow \widetilde{\Omega} B
$$

is a DGA map.

The structure of an $A_{\infty^{-}}$(co)algebra is encoded by the quadratic relations among its operations (also called "higher homotopies"). Although the "direction," i.e., sign, of these higher homotopies is arbitrary, each choice of directions determines a set of signs in the quadratic relations, the "simplest" of which appears on the algebra side when no changes of direction are made; see (7.1) and (7.3) above. Interestingly, the "simplest" set of signs appear on the coalgebra side when $\psi^{n}$ is replaced by $(-1)^{[(n-1) / 2]} \psi^{n}, n \geqslant 1$, i.e., the direction of every third and fourth homotopy is reversed. The choices one makes will depend on the application; for us the appropriate choices are as in (7.3) and (7.5).

Let $\mathcal{A}_{\infty}=\oplus_{n \geqslant 2} C_{*}\left(K_{n}\right)$ and let $\left(A, \varphi^{n}\right)_{n \geqslant 1}$ be an $A_{\infty}$-algebra with quadratic relations as in (7.3). For each $n \geqslant 2$, associate $e^{n-2} \in C_{n-2}\left(K_{n}\right)$ with the operation $\varphi^{n}$ via

$$
e^{n-2} \mapsto(-1)^{n} \varphi^{n}
$$

and each codimension 1 face $d_{(i, \ell)}\left(e^{n-2}\right) \in C_{n-3}\left(K_{n}\right)$ with the quadratic composition

$$
d_{(i, \ell)}\left(e^{n-2}\right) \mapsto \varphi^{n-\ell} \varphi_{i, n-\ell-1-i}^{\ell+1} .
$$

Then (7.7) and (7.8) induce a chain map

$$
\zeta_{A}: \mathcal{A}_{\infty} \longrightarrow \oplus_{n \geqslant 2} \operatorname{Hom}^{*}\left(A^{\otimes n}, A\right)
$$

representing the $A_{\infty}$-algebra structure on $A$. Dually, if $\left(A, \psi^{n}\right)_{n \geqslant 1}$ is an $A_{\infty}$-coalgebra with quadratic relations as in (7.5), the associations

$$
e^{n-2} \mapsto \psi^{n} \text { and } d_{(i, \ell)}\left(e^{n-2}\right) \mapsto \psi_{i, n-\ell-1-i}^{\ell+1} \psi^{n-\ell}
$$

induce a chain map

$$
\xi_{A}: \mathcal{A}_{\infty} \longrightarrow \oplus_{n \geqslant 2} \operatorname{Hom}^{*}\left(A, A^{\otimes n}\right)
$$

representing the $A_{\infty}$-coalgebra structure on $A$. The definition of the tensor product is now immediate:

Definition 30. The tensor product of $A_{\infty}$-algebras $\left(A, \zeta_{A}\right)$ and $\left(B, \zeta_{B}\right)$ is given by

$$
\left(A, \zeta_{A}\right) \otimes\left(B, \zeta_{B}\right)=\left(A \otimes B, \zeta_{A \otimes B}\right),
$$


where $\zeta_{A \otimes B}$ is the sum of the compositions

$$
\begin{aligned}
& C_{*}\left(K_{n}\right) \quad \stackrel{\zeta_{A \otimes B}}{\longrightarrow} \quad H o m\left((A \otimes B)^{\otimes n}, A \otimes B\right) \\
& \Delta_{K} \downarrow \quad \uparrow\left(\sigma_{2, n}\right)^{*} \iota \\
& C_{*}\left(K_{n}\right) \otimes C_{*}\left(K_{n}\right) \underset{\zeta_{A} \otimes \zeta_{B}}{\longrightarrow} \operatorname{Hom}\left(A^{\otimes n}, A\right) \otimes H o m\left(B^{\otimes n}, B\right)
\end{aligned}
$$

over all $n \geqslant 2$; the $A_{\infty}$-algebra operations $\Phi^{n}$ on $A \otimes B$ are given by

$$
\Phi^{n}=\left(\sigma_{2, n}\right)^{*} \iota\left(\zeta_{A} \otimes \zeta_{B}\right) \Delta_{K}\left(e^{n-2}\right) .
$$

Dually, the tensor product of $A_{\infty}$-coalgebras $\left(A, \xi_{A}\right)$ and $\left(B, \xi_{B}\right)$ is given by

$$
\left(A, \xi_{A}\right) \otimes\left(B, \xi_{B}\right)=\left(A \otimes B, \xi_{A \otimes B}\right),
$$

where $\xi_{A \otimes B}$ is the sum of the compositions

$$
\begin{array}{ccc}
C_{*}\left(K_{n}\right) & \stackrel{\xi_{A \otimes B}}{\longrightarrow} & \operatorname{Hom}\left(A \otimes B,(A \otimes B)^{\otimes n}\right) \\
\downarrow & \uparrow\left(\sigma_{n, 2}\right)_{*} \iota \\
\Delta_{*}\left(K_{n}\right) \otimes C_{*}\left(K_{n}\right) & \underset{\xi_{A} \otimes \xi_{B}}{\longrightarrow} & \operatorname{Hom}\left(A, A^{\otimes n}\right) \otimes \operatorname{Hom}\left(B, B^{\otimes n}\right)
\end{array}
$$

over all $n \geqslant 2$; the $A_{\infty}$-coalgebra operations $\Psi^{n}$ on $A \otimes B$ are given by

$$
\Psi^{n}=\left(\sigma_{n, 2}\right)_{*} \iota\left(\xi_{A} \otimes \xi_{B}\right) \Delta_{K}\left(e^{n-2}\right) .
$$

Example 10. If $\left(A, \psi^{n}\right)_{n \geqslant 1}$ is an $A_{\infty}$-coalgebra, the following $A_{\infty}$ operations arise on $A \otimes A$ :

$$
\begin{aligned}
\Psi^{1}= & \psi^{1} \otimes 1+1 \otimes \psi^{1} \\
\Psi^{2}= & \sigma_{2,2}\left(\psi^{2} \otimes \psi^{2}\right) \\
\Psi^{3}= & \sigma_{3,2}\left(\psi_{0}^{2} \psi_{0}^{2} \otimes \psi^{3}+\psi^{3} \otimes \psi_{1}^{2} \psi_{0}^{2}\right) \\
\Psi^{4}= & \sigma_{4,2}\left(\begin{array}{r}
\psi_{0}^{2} \psi_{0}^{2} \psi_{0}^{2} \otimes \psi^{4}+\psi^{4} \otimes \psi_{2}^{2} \psi_{1}^{2} \psi_{0}^{2}+\psi_{0}^{3} \psi_{0}^{2} \otimes \psi_{1}^{2} \psi_{0}^{3} \\
\left.\quad+\psi_{0}^{3} \psi_{0}^{2} \otimes \psi_{1}^{3} \psi_{0}^{2}+\psi_{1}^{2} \psi_{0}^{3} \otimes \psi_{1}^{3} \psi_{0}^{2}-\psi_{0}^{2} \psi_{0}^{3} \otimes \psi_{2}^{2} \psi_{0}^{3}\right)
\end{array}\right.
\end{aligned}
$$

Note that the compositions in Definition 30 only use the operations $\psi^{n}$ and not the quadratic relations (7.5). Indeed, one can iterate an arbitrary family of operations $\left\{\psi^{n}\right\}$ as in Example (10) to produce iterated operations $\Psi^{n}: A^{\otimes k} \rightarrow$ $\left(A^{\otimes k}\right)^{\otimes n}$ whether or not $\left(A, \psi^{n}\right)$ is an $A_{\infty}$-coalgebra. Of course, the $\Psi^{n}$ 's define an $A_{\infty}$-coalgebra structure on $A^{\otimes k}$ whenever $d_{\widetilde{\Omega}\left(A^{\otimes k}\right)}^{2}=0$, and we make extensive use of this fact in the sequel [16]. Finally, since $\Delta_{K}$ is homotopy coassociative (not strict), the tensor product only iterates up to homotopy. In the sequel we always coassociate on the extreme left. 


\section{Appendix}

For completeness, we review the definitions of the functors given by Kadeishvili and Saneblidze in $[\mathbf{8}],[\mathbf{9}]$ from the category of 1-reduced simplicial sets to the category of cubical sets and from the category of 1-reduced cubical sets to the category of permutahedral sets.

\subsection{The cubical set functor $\Omega X$}

Given a 1-reduced simplicial set $X=\left\{X_{n}, \partial_{i}, s_{i}\right\}_{n \geqslant 0}$, define the graded set $\boldsymbol{\Omega} X$ as follows: Let $X^{c}$ be the graded set of formal expressions

$$
X_{n+k}^{c}=\left\{\eta_{i_{k}} \cdots \eta_{i_{1}} \eta_{i_{0}}(x) \mid x \in X_{n}\right\}_{n \geqslant 0 ; k \geqslant 0},
$$

where $\eta_{i_{0}}=1, i_{1} \leqslant \cdots \leqslant i_{k}, 1 \leqslant i_{j} \leqslant n+j-1,1 \leqslant j \leqslant k$, and let $\bar{X}^{c}=s^{-1}\left(X_{>0}^{c}\right)$ be the desuspension of $X^{c}$. Let $\Omega^{\prime} X$ be the free graded monoid generated by $\bar{X}^{c}$; denote elements of $\Omega^{\prime} X$ by $\bar{x}_{1} \cdots \bar{x}_{k}$, where $x_{j} \in X_{m_{j}+1}, m_{j} \geqslant 0$. The total degree $m=\left|\bar{x}_{1} \cdots \bar{x}_{k}\right|=\sum\left|\bar{x}_{j}\right|$ and we write $\bar{x}_{1} \cdots \bar{x}_{k} \in\left(\Omega^{\prime} X\right)_{m}$. The product of two elements $\bar{x}_{1} \cdots \bar{x}_{k}$ and $\bar{y}_{1} \cdots \bar{y}_{\ell}$ is given by concatenation $\bar{x}_{1} \cdots \bar{x}_{k} \bar{y}_{1} \cdots \bar{y}_{\ell}$; the only relation on $\Omega^{\prime} X$ is strict associativity. Let $\Omega X$ be the graded monoid obtained from $\Omega^{\prime} X$ via

$$
\Omega X=\Omega^{\prime} X / \sim,
$$

where $\overline{\eta_{n}(x)} \sim \overline{s_{n}(x)}$ for $x \in X_{>0}$, and $\bar{x}_{1} \cdots \overline{\eta_{m_{i}+1}\left(x_{i}\right)} \cdot \bar{x}_{i+1} \cdots \bar{x}_{k} \sim \bar{x}_{1} \cdots \bar{x}_{i}$. $\overline{\eta_{1}\left(x_{i+1}\right)} \cdots \bar{x}_{k}$ for $x_{i} \in X_{m_{i}+1}^{c}, i<k$. Let $M X$ denote the free monoid generated by $\bar{X}=s^{-1}\left(X_{>0}\right)$; there is an inclusion of graded modules $M X \subset \Omega^{\prime} X$.

Apparently $\Omega^{\prime} X$ canonically admits the structure of a cubical set. Denote the components of Alexander-Whitney diagonal by

$$
\nu_{i}: X_{n} \rightarrow X_{i} \times X_{n-i},
$$

where $\nu_{i}(x)=\partial_{i+1} \cdots \partial_{n}(x) \times \partial_{0} \cdots \partial_{i-1}(x), 0 \leqslant i \leqslant n$, and let $x^{n} \in X_{n}$ denote an $n$-simplex simplex. Then

$$
\nu_{i}\left(x^{n}\right)=\left(x^{\prime}\right)^{i} \times\left(x^{\prime \prime}\right)^{n-i} \in X_{i} \times X_{n-i}
$$

for all $n>0$. For $1 \leqslant i \leqslant n-1$, define face operators $d_{i}^{0}, d_{i}^{1}:(\boldsymbol{\Omega} X)_{n-1} \rightarrow(\boldsymbol{\Omega} X)_{n-2}$ on a (monoidal) generator $\overline{x^{n}} \in \bar{X}_{n} \subset \bar{X}_{n}^{c}$ by

$$
d_{i}^{0}\left(\overline{x^{n}}\right)=\overline{\left(x^{\prime}\right)^{i}} \cdot \overline{\left(x^{\prime \prime}\right)^{n-i}} \text { and } d_{i}^{1}\left(\overline{x^{n}}\right)=\overline{\partial_{i}\left(x^{n}\right)},
$$

and extend to elements $\bar{x}_{1} \cdots \bar{x}_{k} \in M X$ via

$$
\begin{aligned}
& d_{i}^{0}\left(\bar{x}_{1} \cdots \bar{x}_{k}\right)=\bar{x}_{1} \cdots \overline{\left(x_{q}^{\prime}\right)^{j_{q}}} \cdot \overline{\left(x_{q}^{\prime \prime}\right)^{m_{q}-j_{q}+1}} \cdots \bar{x}_{k}, \\
& d_{i}^{1}\left(\bar{x}_{1} \cdots \bar{x}_{k}\right)=\bar{x}_{1} \cdots \overline{\partial_{j_{q}}\left(x_{q}\right)} \cdots \bar{x}_{k},
\end{aligned}
$$

where $i=m_{(q-1)}+j_{q} \leqslant m_{(q)}, 1 \leqslant i \leqslant n-1,1 \leqslant q \leqslant k$. Then the defining identities for a cubical set involving $d_{i}^{0}$ and $d_{i}^{1}$ can easily be checked on $M X$. In particular, the simplicial relations between the $\partial_{i}$ 's imply the cubical relations between $d_{i}^{1}$ 's; the associativity relations between $\nu_{i}$ 's imply the cubical relations between $d_{i}^{0}$ 's, and the commutativity relations between $\partial_{i}$ 's and $\nu_{j}$ 's imply the cubical relations 
between $d_{i}^{1}$ 's and $d_{j}^{0}$ 's. Next, define degeneracy operators $\eta_{i}:(\boldsymbol{\Omega} X)_{n-1} \rightarrow(\boldsymbol{\Omega} X)_{n}$ on a (monoidal) generator $\bar{x} \in\left(\bar{X}^{c}\right)_{n-1}$ by

$$
\eta_{i}(\bar{x})=\overline{\eta_{i}(x)}
$$

and extend to elements $\bar{x}_{1} \cdots \bar{x}_{k} \in \boldsymbol{\Omega} X$ via

$$
\begin{aligned}
& \eta_{i}\left(\bar{x}_{1} \cdots \bar{x}_{k}\right)=\bar{x}_{1} \cdots \eta_{j_{q}}\left(\overline{x_{q}}\right) \cdots \bar{x}_{k}, \\
& \eta_{n}\left(\bar{x}_{1} \cdots \bar{x}_{k}\right)=\bar{x}_{1} \cdots \bar{x}_{m_{k-1}} \cdot \eta_{m_{k}+1}\left(\bar{x}_{k}\right),
\end{aligned}
$$

where $i=m_{(q-1)}+j_{q} \leqslant m, 1 \leqslant i \leqslant n-1,1 \leqslant q \leqslant k$, and extend face operators on degenerate elements inductively so that the defining identities of a cubical set are satisfied. Then in particular, the following identities hold for all $x^{n} \in X_{n}$ :

$$
\begin{aligned}
& d_{1}^{0}\left(\overline{x^{n}}\right)=\overline{\left(x^{\prime}\right)^{1}} \cdot \overline{\left(x^{\prime \prime}\right)^{n-1}}=e \cdot \overline{\left(x^{\prime \prime}\right)^{n-1}}=\overline{\left(x^{\prime \prime}\right)^{n-1}}=\overline{\partial_{0}\left(x^{n}\right)}, \\
& d_{n-1}^{0}\left(\overline{x^{n}}\right)=\overline{\left(x^{\prime}\right)^{n-1}} \cdot \overline{\left(x^{\prime \prime}\right)^{1}}=\overline{\left(x^{\prime \prime}\right)^{n-1}} \cdot e=\overline{\left(x^{\prime}\right)^{n-1}}=\overline{\partial_{n}\left(x^{n}\right),}
\end{aligned}
$$

where $e \in(\boldsymbol{\Omega} X)_{0}$ denotes the unit. It is easy to see that the cubical set $\left\{\boldsymbol{\Omega} X, d_{i}^{0}, d_{i}^{1}\right.$, $\left.\eta_{i}\right\}$ depends functorially on $X$.

\subsection{The permutahedral set functor $\Omega Q$}

Let $Q=\left(Q_{n}, d_{i}^{0}, d_{i}^{1}, \eta_{i}\right)_{n \geqslant 0}$ be a 1 -reduced cubical set. Recall that the diagonal

$$
\Delta: C_{*}(Q) \rightarrow C_{*}(Q) \otimes C_{*}(Q)
$$

on $C_{*}(Q)$ is defined on $a \in Q_{n}$ by

$$
\Delta(a)=\Sigma(-1)^{\epsilon} d_{B}^{0}(a) \otimes d_{A}^{1}(a),
$$

where $d_{B}^{0}=d_{j_{1}}^{0} \cdots d_{j_{q}}^{0}, d_{A}^{1}=d_{i_{1}}^{1} \cdots d_{i_{p}}^{1}$, summation is over all shuffles $(A ; B)=$ $\left(i_{1}<\cdots<i_{q} ; j_{1}<\cdots<j_{p}\right)$ of $\underline{n}$ and $\epsilon$ is the sign of the shuffle. The primitive components of the diagonal are given by the extreme cases $A=\varnothing$ and $B=\varnothing$.

Let $\bar{Q}=s^{-1}\left(Q_{>0}\right)$ denote the desuspension of $Q$, let $\Omega^{\prime \prime} Q$ be the free graded monoid generated by $\bar{Q}$ with the unit $e \in \bar{Q}_{1} \subset \boldsymbol{\Omega}^{\prime \prime} Q$ and let $\Upsilon$ be the set of formal expressions

$$
\Upsilon=\left\{\varrho_{M_{k} \mid N_{k}}\left(\left(\cdots \varrho_{M_{2} \mid N_{2}}\left(\varrho_{M_{1} \mid N_{1}}\left(\bar{a}_{1} \cdot \bar{a}_{2}\right) \cdot \bar{a}_{3}\right) \cdots\right) \cdot \bar{a}_{k+1}\right) \mid a_{i} \in Q_{r_{i}}\right\}_{r_{i} \geqslant 1 ; k \geqslant 2},
$$

$M_{i} \mid N_{i} \in \mathcal{P}_{r_{(i)}, r_{i+1}}\left(r_{(i+1)}\right)$ or $M_{i} \mid N_{i} \in \mathcal{P}_{r_{i+1}, r_{(i)}}\left(r_{(i+1)}\right), 1 \leqslant i \leqslant k$. Note that one or more of the $a_{i}$ 's can be the unit $e$. Adjoin the elements of $\Upsilon$ to $\Omega^{\prime \prime} Q$ and obtain the graded monoid $\Omega^{\prime} Q$ and let $\Omega Q$ be the monoid

$$
\Omega Q=\Omega^{\prime} Q / \sim
$$

where $\varrho_{M \mid N}(\bar{a} \cdot \bar{b}) \sim \varrho_{N \mid M}(\bar{a} \cdot \bar{b}), \varrho_{j \mid \underline{n} \backslash j}(e \cdot \bar{a}) \sim \varrho_{\underline{n} \backslash j \mid j}(\bar{a} \cdot e) \sim \overline{\eta_{j}(a)}, a, b \in Q_{>0}$, and $\bar{a}_{1} \cdots \varrho_{r_{i} \mid r_{i}+1}\left(\bar{a}_{i} \cdot e\right) \cdot \bar{a}_{i+2} \cdots \bar{a}_{k+1} \sim \bar{a}_{1} \cdots \bar{a}_{i} \cdot \varrho_{1 \mid r_{i+2}+1 \backslash 1}\left(e \cdot \bar{a}_{i+2}\right) \cdots \bar{a}_{k+1}$ for $a_{i} \in Q_{r_{i}}, a_{i+1}=e, 1 \leqslant i \leqslant k$. Then $\Omega Q$ is canonically a multipermutahedral set in the following way: First, define the face operator $d_{A \mid B}$ on a monoidal generator $\bar{a} \in \bar{Q}_{n}$ by

$$
d_{A \mid B}(\bar{a})=\overline{d_{B}^{0}(a)} \cdot \overline{d_{A}^{1}(a)}, \quad A \mid B \in \mathcal{P}_{*, *}(n) .
$$


Next, use the formulas in the definition of a singular multipermutahedral set (4.3) to define $d_{A \mid B}$ and $\varrho_{M \mid N}$ on decomposables. In particular, the following identities hold for $1 \leqslant i \leqslant n$ :

$$
d_{i \mid \underline{n+1} \backslash i}(\bar{a})=\overline{d_{i}^{1}(a)} \text { and } d_{\underline{n+1} \backslash i \mid i}(\bar{a})=\overline{d_{i}^{0}(a)} .
$$

It is easy to see that $\left(\Omega Q, d_{A \mid B}, \varrho_{M \mid N}\right)$ is a multipermutahedral set that depends functorially on $Q$.

Remark 3. The fact that the definition of $\Omega Q$ uses all cubical degeneracies is justified geometrically by the fact that a degenerate singular $n$-cube in the base of a path space fibration lifts to a singular $(n-1)$-permutahedron in the fibre, which is degenerate with respect to Milgram's projections [14] (c.f., the definition of the cubical set $\Omega X$ on a simplicial set $X)$.

\section{References}

[1] J. F. Adams, On the cobar construction, Proc. Nat. Acad. Sci. (USA), 42 (1956), 409-412.

[2] H. J. Baues, The cobar construction as a Hopf algebra, Invent. Math., 132 (1998), 467-489.

[3] G. Carlsson and R. J. Milgram, Stable homotopy and iterated loop spaces, Handbook of Algebraic Topology (Edited by I. M. James), North-Holland (1995), 505-583.

[4] H.S.M. Coxeter, W.O.J. Moser, Generators and relations for discrete groups, Springer-Verlag, 1972.

[5] Matthias R. Gaberdiel and Barton Zwiebach, Tensor constructions of open string theories I: Foundations, Nucl.Phys. B505 (1997), 569-624.

[6] N. Iwase and M. Mimura, Higher homotopy associativity, Lecture Notes in Math., 1370 (1986), 193-220.

[7] D. W. Jones, A general theory of polyhedral sets and corresponding Tcomplexes, Dissertationes Mathematicae, CCLXYI, Warszava (1988).

[8] T. Kadeishvili and S. Saneblidze, A cubical model of a fibration, preprint, AT/0210006.

[9] tion, preprint, AT/0210224.

[10] D. M. Kan, Abstract homotopy I, Proc. Nat. Acad. Sci. U.S.A., 41 (1955), 1092-1096.

[11] T. Lada and M. Markl, Strongly homotopy Lie algebras, Communications in Algebra 23 (1995), 2147-2161.

[12] J.-L. Loday and M. Ronco, Hopf algebra of the planar binary trees, Adv. in Math. 139, No. 2 (1998), 293-309.

[13] S. Mac Lane, "Homology," Springer-Verlag, Berlin/New York, 1967.

[14] R. J. Milgram, Iterated loop spaces, Ann. of Math., 84 (1966), 386-403. 
[15] S. Saneblidze and R. Umble, A diagonal on the associahedra, preprint, math. AT/0011065.

[16] , The biderivative and $A_{\infty}$-bialgebras, J. Homology, Homotopy and Appl., to appear, preprint, math. AT/0011065.

[17] J. R. Smith, "Iterating the cobar construction", Memiors of the Amer. Math. Soc. 109, Number 524, Providence, RI, 1994.

[18] J. D. Stasheff, Homotopy associativity of $H$-spaces I, II, Trans. Amer. Math. Soc., 108 (1963), 275-312.

[19] A. Tonks, Relating the associahedron and the permutohedron, In "Operads: Proceedings of the Renaissance Conferences (Hartford CT / Luminy Fr 1995)" Contemporary Mathematics, 202 (1997), pp. 33-36.

[20] G. Ziegler, "Lectures on Polytopes," GTM 152, Springer-Verlag, New York, 1995.

This article may be accessed via WWW at http://www.rmi.acnet.ge/hha/ or by anonymous ftp at

ftp://ftp.rmi.acnet.ge/pub/hha/volumes/2004/n1a20/v6n1a20.(dvi,ps,pdf)

Samson Saneblidze sane@rmi.acnet.ge

A. Razmadze Mathematical Institute

Georgian Academy of Sciences

M. Aleksidze st., 1

0193 Tbilisi, Georgia

Ronald Umble ron.umble@millersville.edu

Department of Mathematics

Millersville University of Pennsylvania

Millersville, PA. 17551 